\title{
Fixed point theorems in modular $G$-metric spaces
}

\author{
Godwin Amechi Okeke ${ }^{1 *}$ D, Daniel Francis ${ }^{1}$, Manuel de la Sen² and Mujahid Abbas ${ }^{3,4}$
}

"Correspondence:
gaokeke1@yahoo.co.uk;
godwin.okeke@futo.edu.ng
${ }^{1}$ Functional Analysis and
Optimization Research Group
Laboratory (FANORG), Department
of Mathematics, School of Physical
Sciences, Federal University of
Technology Owerri, P.M.B. 1526,
Owerri, Imo State, Nigeria
Full list of author information is
available at the end of the article

available at the end of the article

\section{Springer}

\begin{abstract}
We prove the existence and uniqueness of fixed points of some generalized contractible operators defined on modular G-metric spaces and also prove the modular G-continuity of such operators. Furthermore, we prove that some generalized weakly compatible contractive operators in modular G-metric spaces have a unique fixed point. Our results extend, generalize, complement and include several known results as special cases.

MSC: 30Lxx; 32A70; 47H10; 46A80; 46E30; 54H25; 55M20; 58C30

Keywords: Fixed point; Modular G-metric spaces; Modular G-continuity; Operators; Contractive mapping; Weakly compatible; $\Delta_{3}$-type condition; Existence and uniqueness
\end{abstract}

\section{Introduction}

Banach in 1922 [16] proved his famous contraction mapping principle in a complete metric space. Since then this result of Banach has been characterized in different spaces such as those in quasi-metric, multiplicative metric space, $b$-metric, $D$-metric space, $D^{*}$-metric spaces, $G$-metrics, $F$-metric spaces, modular and modular $G$-metric spaces. Some other results in $b$-metric space and control metric space can be found in $[33,64]$ and the references therein and $[6,56]$.

In 1966, Gahler in [24], introduced 2-metric spaces, and Dhage in [23] extended the work in [24] in which $D$-metric spaces were introduced. These authors claimed that their results generalized the concept of metric spaces. The nonnegative real function $D$ is called a $D$-metric on $X$. The set $X$ together with such a generalized metric $D$ is called a generalized metric space, or $D$-metric space, and denoted by $(X, D)$ and some of their topological properties have been studied by Dhage.

The concept of G-metric space was introduced by Mustafa and Sims in [45] and they pointed out that the fundamental topology properties of $D$-metric spaces introduced by Dhage were incorrect. To ameliorate the drawback about $D$-metric spaces, Mustafa and Sims in [46] introduced a generalization of metric spaces, which they called G-metric spaces and proved some fixed point theorems. Samet et al. [62] and Jleli-Samet [29] noticed that some fixed point theorems in the context of a $G$-metric space in the literature can be deduced directly from some existing results in the setting of a quasi-metric and metric

(c) The Author(s) 2021. This article is licensed under a Creative Commons Attribution 4.0 International License, which permits use sharing, adaptation, distribution and reproduction in any medium or format, as long as you give appropriate credit to the original author(s) and the source, provide a link to the Creative Commons licence, and indicate if changes were made. The images or other third party material in this article are included in the article's Creative Commons licence, unless indicated otherwise in a credit line to the material. If material is not included in the article's Creative Commons licence and your intended use is not permitted by statutory regulation or exceeds the permitted use, you will need to obtain permission directly from the copyright holder. To view a copy of this licence, visit http://creativecommons.org/licenses/by/4.0/. 
space. In fact, if the contraction condition of the fixed point theorem on a $G$-metric space can be reduced to two variables instead of three variables, then one can construct an equivalent fixed point theorems in the setup of a usual metric space. Precisely, in $[29,62]$ the authors noticed that $2 d(x, y)=G(x, y, y)$ forms a quasi-metric. Hence, if one can transform the contraction condition of existence results in a $G$-metric space in such terms, $G(x, y, y)$, then the related fixed point results become the known fixed point results in the context of a quasi-metric space. Sequel to these arguments about the concept of generalized metric space, Karapinar and Agarwal [30] noticed that the techniques used in [29,62] are valid if the contraction condition in the statement of the theorem(s) can be reduced into two variables. Furthermore, Karapinar and Agarwal [30] proved some fixed point theorems in the context of a $G$-metric space for which the techniques used in $[29,62]$ are inapplicable.

The reduction method introduced by Samet et al. [62] and Jleli and Samet [29] reported that most of fixed point results in the context of $G$-metric space, defined by Mustafa and Sims, can be derived from the usual fixed point theorems on the usual metric space. This enabled [12] et al. to prove some fixed point theorems in the framework of $G$-metric space that contradicted the ideas of Samet et al. [62] and Jleli and Samet [29] showing that not all fixed point results can be obtained from the existence results in the context of associated metric space.

Shatanawi et al. [63] utilized the concept of $\Omega$-distance in the sense of Saadati to introduce new fixed and common fixed point results for mappings of cyclic form, through the concept of G-metric space in sense of Mustafa and Sims [46]. Shatanawi et al. [63] pointed out that the method of Jleli and Samet cannot be applied to their results. Some authors have obtained some fixed point theorems in the structure of G-metric spaces and established that they cannot be obtained from the existing results in the context of allied metric spaces and do not meet the remarks of Samet et al. [62] and Jleli and Samet [29]. Other interesting results about fixed point theorems in $G$-metric spaces can be found in $[1-3,7,9,10,25,32,39-41,43,44,47,48,65]$ while [38] investigated common fixed points of weakly compatible mappings in $G$-metric spaces.

Mustafa and Jaradat in their recent paper [42] produced an example to show that $D^{*}$ metric need not be $G$-metric as well as the $G$-metric need not be $D^{*}$-metric. With all these results, in this paper, we will give some results in the setting of modular $G$-metric which cannot be reduced to its allied modular metric spaces.

Modular function space and its theory were introduced by Nakano in [51] in line with the theory of order spaces. Musielak and Orlicz redefined and generalized the concept in $[36,57]$ and proved some fixed point theorems. The notion of a modular metric on an arbitrary set and the corresponding modular space, which is more general than a metric space, were introduced and studied in a landmark paper by Chistyakov in [20]. Also fixed point theorems in modular metric spaces and their applications have been dealt with in [21] and [22]. Many authors have introduced and generalized, studied fixed point theorems and their applications in modular metric spaces such as those in $[4,5,11,13,17,18,20,21,27,28,34,35,49,54,61]$ in modular metric spaces; see $[13,18,21,52,55]$ and the references therein and some other interesting results of fixed point theorems in metric spaces appearing in $[7,8,16,17,26,31,34,37,50,58,59,61]$ and [60].

Since then, many authors such as $[4,5,11,13,18,19,27,28,35,49]$ have studied and improved some results of fixed point theory in the setting of modular metric spaces, while 
$[52,55]$ studied iterative approximation of fixed point of multi-valued $\rho$-quasi-contractive and multi-valued $\rho$-quasi-nonexpansive mappings in modular function spaces, respectively.

Following the notion of modular metric space in [5], Definition 1.1 in [14] can be interpreted in terms of modular metric spaces as follows. Let $X$ be a nonempty set, and let $\omega^{G}:(0, \infty) \times X \times X \times X \rightarrow[0, \infty]$ be a function defined by $\omega_{\lambda}^{G}(x, y, z)=\omega_{\lambda}(x, y)+\omega_{\lambda}(y, z)+$ $\omega_{\lambda}(x, z)$ for all $x, y, z \in X$ and $\lambda>0$. Then $\left(X, \omega^{G}\right)$ is a modular $G$-metric space. In this case, for all $\lambda \in(0, \infty), \omega_{\lambda}^{G}(x, y, z)$ can be interpreted as the perimeter of the triangle of vertices $x, y$ and $z$. Now axiom (1) means that with one point we cannot have a positive perimeter for all $\lambda \in(0, \infty)$, and axiom (2) is equivalent to the fact that the distance between two different points can never be zero for all $\lambda \in(0, \infty)$. Meanwhile, as the perimeter of a triangle for all $\lambda \in(0, \infty)$ cannot depend on the order in which we consider its vertices, we have axiom (4) and axiom (5) is an extension of the triangle inequality using a fourth vertex for all $\lambda \in(0, \infty)$. By axiom (3), we see that the length of an edge of a triangle is less than or equal to its semi-perimeter, i.e. $\omega_{\lambda}(x, y) \leq \frac{\omega_{\lambda}(x, y)+\omega_{\lambda}(y, z)+\omega_{\lambda}(x, z)}{2}$ for all $x, y, z \in X$ and $\lambda>0$, which is the famous Hero formula.

In 2013, the concept of modular G-metric spaces were introduced by Azadifar et al. in [14]. They obtained some fixed point theorems of contractive mappings defined on modular G-metric spaces. The existence of fixed point of contractive mapping defined on modular G-metric spaces was proved, where the completeness is replaced with weaker conditions.

Azadifar et al. [15], used the theory they developed in [14] to prove the existence and uniqueness of common fixed point of a pair of weakly compatible mappings satisfying the $\Phi$-map in modular G-metric spaces.

Very recently, Okeke and Francis [53] proved the existence and uniqueness of a fixed point of mappings satisfying Geraghty-type contractions in the setting of preordered modular G-metric spaces. The results were applied in solving nonlinear Volterra-Fredholmtype integral equations.

In the present paper, we introduce the concept of $\Delta_{3}$-type condition in modular Gmetric spaces. We will prove the existence and uniqueness of fixed point of some generalized contractible operators defined on modular $G$-metric spaces satisfying a $\Delta_{3}$-type condition and also prove the modular G-continuity of such operators in modular G-metric spaces. Our results extend, generalize, complement and include several known results as special cases, including the results of [14] and [15].

\section{Preliminaries}

Here we shall define the modular G-metric space following Azadifar et al. [14].

Definition 2.1 ([14]) Let $X$ be a nonempty set, and let $\omega^{G}:(0, \infty) \times X \times X \times X \rightarrow[0, \infty]$ be a function satisfying;

(1) $\omega_{\lambda}^{G}(x, y, z)=0$ for all $x, y, z \in X$ and $\lambda>0$ if $x=y=z$,

(2) $\omega_{\lambda}^{G}(x, x, y)>0$ for all $x, y \in X$ and $\lambda>0$ with $x \neq y$,

(3) $\omega_{\lambda}^{G}(x, x, y) \leq \omega_{\lambda}^{G}(x, y, z)$ for all $x, y, z \in X$ and $\lambda>0$ with $z \neq y$,

(4) $\omega_{\lambda}^{G}(x, y, z)=\omega_{\lambda}^{G}(x, z, y)=\omega_{\lambda}^{G}(y, z, x)=\cdots$ for all $\lambda>0$ (symmetry in all three variables),

(5) $\omega_{\lambda+\mu}^{G}(x, y, z) \leq \omega_{\lambda}^{G}(x, a, a)+\omega_{\mu}^{G}(a, y, z)$, for all $x, y, z, a \in X$ and $\lambda, v>0$, 
then the function $\omega_{\lambda}^{G}(\cdot, \cdot)$ is called a modular $G$-metric on $X$ and the pair $\left(X, \omega^{G}\right)$ is called a modular G-metric space.

Definition 2.2 Let $X$ be a nonempty set, and let $\omega^{G}:(0, \infty) \times X \times X \times X \rightarrow[0, \infty]$ is said to be convex modular (G-metric) on a set $X$ if it satisfies the conditions (1) and (4) of the Definition 2.1 as well as the axiom $\omega_{\lambda+\mu}^{G}(x, y, z) \leq \frac{\lambda}{\lambda+\mu} \omega_{\lambda}^{G}(x, a, a)+\frac{\mu}{\lambda+\mu} \omega_{\mu}^{G}(a, y, z)$, for all $\lambda>0, \mu>0$ and $x, y, z, a \in X_{\omega^{G}}$.

Definition 2.3 Let $X$ be a nonempty set, and let $\omega_{\lambda}^{G}:(0, \infty) \times X \times X \times X \rightarrow[0, \infty]$ be a function, then it is non-symmetric if $\omega_{\lambda}^{G}$ is not symmetric, for all $\lambda>0$.

\section{Remark 2.1}

(a) If $x=a$, then condition (5) of Definition 2.1 above becomes $\omega_{\lambda+\mu}^{G}(a, y, z) \leq \omega_{\mu}^{G}(a, y, z)$.

(b) Condition (5) of Definition 2.1 is called the rectangle inequality.

In this paper, we will take $X_{\omega} G$ to be a modular G-metric space.

Definition 2.4 ([14]) Let $\left(X, \omega^{G}\right)$ be a modular $G$-metric space. The sequence $\left\{x_{n}\right\}_{n \in \mathbb{N}}$ in $X_{\omega^{G}}$ is modular $G$-convergent to $x$, if it converges to $x$ in the topology $\tau\left(\omega_{\lambda}^{G}\right)$.

A function $T: X_{\omega^{G}} \rightarrow X_{\omega^{G}}$ at $x \in X_{\omega^{G}}$ is called modular $G$-continuous if $\omega_{\lambda}^{G}\left(x_{n}, x, x\right) \rightarrow 0$ then $\omega_{\lambda}^{G}\left(T x_{n}, T x, T x\right) \rightarrow 0$, for all $\lambda>0$.

Remark 2.2 We see that a function $T: X_{\omega^{G}} \rightarrow X_{\omega^{G}}$ at $x \in X_{\omega^{G}}$ is called modular Gcontinuous if $\omega_{\lambda}^{G}\left(x, x_{n}, x_{n}\right) \rightarrow 0$ then $\omega_{\lambda}^{G}\left(T x, T x_{n}, T x_{n}\right) \rightarrow 0$, for all $\lambda>0$.

Definition 2.5 ([14]) Let $\left(X, \omega^{G}\right)$ be a modular $G$-metric space, then the sequence $\left\{x_{n}\right\}_{n \in \mathbb{N}} \subseteq X_{\omega^{G}}$ is said to be modular G-Cauchy if for every $\epsilon>0$, there exists $n_{\epsilon} \in \mathbb{N}$ such that $\omega_{\lambda}^{G}\left(x_{n}, x_{m}, x_{l}\right)<\epsilon$ for all $n, m, l \geq n_{\epsilon}$ and $\lambda>0$.

A modular $G$-metric space $X_{\omega^{G}}$ is said to be modular $G$-complete if every modular GCauchy sequence in $X_{\omega^{G}}$ is modular $G$-convergent in $X_{\omega^{G}}$.

Definition 2.6 ([15]) Let $g$ and $h$ be single-valued self-mappings on a set $X$. If $w=g x=h x$ for some $x \in X$, then $x$ is called a coincidence point of $g$ and $h$, and $w$ is called a point of coincidence of $g$ and $h$.

Definition 2.7 ([15]) A pair of maps $g$ and $h$ is called weakly compatible pair if they commute at coincidence point.

Definition 2.8 ([31]) Let $T, S$ be two self-mappings on a nonempty set $X$. Then:

(i) $x \in X$ is called a fixed point of $T$ if $T x=x$.

(ii) $x \in X$ is called a coincidence point of $T$ and $S$ if $T x=S x$.

(iii) $x \in X$ is called a common fixed point of $T$ and $S$ if $T x=S x=x$.

(iv) $x \in X$ is called a commuting point of $T, L$ if $T L x=L T x$.

Following [4], we give the definition of being modular G-bounded as follows.

Definition 2.9 We say that $M \subseteq X_{\omega^{G}}$ is G-bounded provided that $\delta_{\omega^{G}}(M)=$ $\sup \left\{\omega_{\lambda}^{G}(a, b, c) ; a, b, c \in M<\infty\right\}$. 
Proposition 2.1 ([15]) Let $g$ and $h$ be weakly compatible self-mappings on a set $X$. If $g$ and $h$ have a unique point of coincidence $w=g x=h x$, then $w$ is the unique common fixed point of $g$ and $h$.

Remark 2.3 Observe that, if $\lim _{n \rightarrow \infty} \omega_{\alpha}^{G}\left(x_{n}, x_{n}, x\right)=0$, for some $\alpha>0$, then $\lim _{n \rightarrow \infty} \omega_{\alpha}^{G}\left(x_{n}\right.$, $\left.x_{n}, x\right)=0$ may not necessarily hold for all $\alpha>0$.

Definition 2.10 Let $\left(X, \omega^{G}\right)$ be a modular G-metric space, we say that $\omega$ satisfy the $\Delta_{3}$ condition if $\lim _{n \rightarrow \infty} \omega_{\alpha}^{G}\left(x_{n}, x_{n}, x\right)=0$, for some $\alpha>0$ implies that $\lim _{n \rightarrow \infty} \omega_{\alpha}^{G}\left(x_{n}, x_{n}, x\right)=0$ for all $\alpha>0, x_{n} \subseteq X_{\omega^{G}}$.

We next introduce the $\Delta_{3}$-type condition which will play a crucial role in the proofs of our results in this paper.

Definition 2.11 Let $\left(X, \omega^{G}\right)$ be a modular $G$-metric space, for all $\lambda>0$, we say that $\omega$ satisfies a $\Delta_{3}$-type condition if for $\alpha>0$, there exists $C_{\alpha}>0$ such that $\omega_{\frac{\lambda}{\alpha}}^{G}(x, y, z) \leq C_{\alpha} \omega_{\lambda}^{G}(x, y, z)$, for all $x, y, z \in X$ and for any $\lambda>0$ and $x$ is distinct from $y, z$.

Remark 2.4 If $\alpha=2$, then $\omega_{\frac{\lambda}{2}}^{G}(x, y, z) \leq C_{2} \omega_{\lambda}^{G}(x, y, z)$, for some $C_{2}>0$.

Proposition $2.2([14])$ Let $\left(X, \omega^{G}\right)$ be a modular G-metric space, for any $x, y, z, a \in X$, it follows that:

(1) If $\omega_{\lambda}^{G}(x, y, z)=0$ for all $\lambda>0$, then $x=y=z$.

(2) $\omega_{\lambda}^{G}(x, y, z) \leq \omega_{\frac{\lambda}{2}}^{G}(x, x, y)+\omega_{\frac{\lambda}{2}}^{G}(x, x, z)$ for all $\lambda>0$.

(3) $\omega_{\lambda}^{G}(x, y, y) \leq 2 \omega_{\frac{\lambda}{2}}^{G}(x, x, y)$ for all $\lambda>0$.

(4) $\omega_{\lambda}^{G}(x, y, z) \leq \omega_{\frac{\lambda}{2}}^{G}(x, a, z)+\omega_{\frac{\lambda}{2}}^{G}(a, y, z)$ for all $\lambda>0$.

(5) $\omega_{\lambda}^{G}(x, y, z) \leq \frac{2}{3}\left(\omega_{\frac{\lambda}{2}}^{G}(x, y, a)+\omega_{\frac{\lambda}{2}}^{G}(x, a, z)+\omega_{\frac{\lambda}{2}}^{G}(a, y, z)\right)$ for all $\lambda>0$.

(6) $\omega_{\lambda}^{G}(x, y, z) \leq \omega_{\frac{\lambda}{2}}^{G}(x, a, a)+\omega_{\frac{\lambda}{4}}^{G}(y, a, a)+\omega_{\frac{\lambda}{4}}^{G}(z, a, a)$ for all $\lambda>0$.

Proposition 2.3 ([14]) Let $\left(X, \omega^{G}\right)$ be a modular G-metric space and $\left\{x_{n}\right\}_{n \in \mathbb{N}}$ be a sequence in $X$. Then the following are equivalent:

(1) $\left\{x_{n}\right\}_{n \in \mathbb{N}}$ is $\omega^{G}$-convergent to $x$,

(2) $\omega_{\lambda}\left(x_{n}, x\right) \rightarrow 0$ as $n \rightarrow \infty$, i.e. $\left\{x_{n}\right\}_{n \in \mathbb{N}}$ converges to $x$ relative to the modular metric $\omega_{\lambda}$,

(3) $\omega_{\lambda}^{G}\left(x_{n}, x_{n}, x\right) \rightarrow 0$ as $n \rightarrow \infty$ for all $\lambda>0$,

(4) $\omega_{\lambda}^{G}\left(x_{n}, x, x\right) \rightarrow 0$ as $n \rightarrow \infty$ for all $\lambda>0$,

(5) $\omega_{\lambda}^{G}\left(x_{m}, x_{n}, x\right) \rightarrow 0$ as $m, n \rightarrow \infty$ for all $\lambda>0$.

\section{Main results}

We state our main results as follows.

Theorem 3.1 Let $\left(X, \omega^{G}\right)$ be a G-complete non-symmetric modular G-metric space satisfying a $\Delta_{3}$-type condition, such that $C_{2} C_{4} \in\left(0, \frac{1}{4 \rho}\right)$ and let $T: X_{\omega^{G}} \rightarrow X_{\omega^{G}}$ be a mapping. Suppose that there exists $\lambda>0$ and $\rho \in(0,1)$ such that

$$
\omega_{\lambda}^{G}(T x, T y, T z) \leq \rho K_{\lambda}(x, y, z) \quad \text { for all } x, y, z \in X_{\omega^{G}}, \lambda>0
$$


where

$$
\begin{aligned}
& K_{\lambda}(x, y, z) \\
& =\left\{\begin{array}{l}
\omega_{\lambda}^{G}(x, y, z), \omega_{\lambda}^{G}(x, z, T y), \omega_{\lambda}^{G}(T x, y, z), \omega_{\lambda}^{G}(x, T x, y), \omega_{\lambda}^{G}(y, z, T y), \\
\omega_{\lambda}^{G}(x, T x, T x), \omega_{\lambda}^{G}(x, T y, T y), \frac{\omega_{\lambda}^{G}(y, z, T y)\left[1+\omega_{\lambda}^{G}(x, T x, y)\right]}{1+\omega_{\lambda}^{G}(x, y, z)}, \\
\omega_{\lambda}^{G}(x, T x, z), \omega_{\lambda}^{G}(y, T y, T y), \frac{\omega_{\lambda}^{G}(x, T y, z)\left[1+\omega_{\lambda}^{G}(x, T x, y)\right]}{1+\omega_{\lambda}^{G}(x, y, z)}, \omega_{\lambda}^{G}(y, T x, T y), \\
\frac{\omega_{\lambda}^{G}(x, T y, z)\left[1+\omega_{\lambda}^{G}(x, y, z)\right]}{1+\omega_{\lambda}^{G}(x, y, z)+\omega_{\lambda}^{G}(y, T x, z)}, \omega_{\lambda}^{G}\left(y, T^{2} x, T y\right), \\
\omega_{\lambda}^{G}(y, T z, T z), \frac{\omega_{\lambda}^{G}(x, T y, z)\left[1+\omega_{\lambda}^{G}(x, y, T y)\right]}{1+\omega_{\lambda}^{G}(T x, y, z)+\omega_{\lambda}^{G}(x, z, T y)}, \omega_{\lambda}^{G}(z, T x, T x), \\
\omega_{\lambda}^{G}(z, T z, T z), \omega_{\lambda}^{G}(z, T x, T y), \omega_{\lambda}^{G}\left(z, T^{2} x, T z\right), \omega_{\lambda}^{G}\left(T x, T^{2} x, T y\right), \\
\omega_{\lambda}^{G}\left(T x, T^{2} x, T z\right), \frac{\omega_{\lambda}^{G}(x, z, T y)\left[1+\omega_{\lambda}^{G}(x, z, T y)\right]}{1+\omega_{\lambda}^{G}(T x, y, z)+\omega_{\lambda}^{G}(x, z, T y)}, \\
\frac{\omega_{\lambda}^{G}\left(T^{2} x, T y, T z\right)\left[1+\omega_{\lambda}^{G}(T x, y, z)+\omega_{\lambda}^{G}(T x, y, T z)\right]}{1+\omega_{\lambda}^{G}(T x, y, T z)+\omega_{\lambda}^{G}\left(T^{2} x, T y, T z\right)}
\end{array}\right\} .
\end{aligned}
$$

Then $T$ has a unique fixed point in $X_{\omega^{G}}$ and is modular G-continuous at the fixed point (say u).

Proof If $x=y=z \in X_{\omega^{G}}$, then, for $\lambda>0, \omega_{\lambda}^{G}(T x, T x, T x) \leq \rho K_{\lambda}(x, x, x)$. But $K_{\lambda}(x, x, x) \geq 0$ implies that $0 \leq \rho K_{\lambda}(x, x, x)$, so that $K_{\lambda}(x, x, x) \geq 0$ and $\rho \neq 0$. Suppose that $K_{\lambda}(x, x, x)=0$, then there is nothing to prove because, $T x=x$. In fact, $\omega_{\lambda}^{G}(x, x, x)=0$. This is condition (1) of Definition 2.1, for $\lambda>0$, so that

$$
\begin{aligned}
& K_{\lambda}(x, x, x) \\
& =\max \left\{\begin{array}{l}
\omega_{\lambda}^{G}(x, x, x), \omega_{\lambda}^{G}(x, x, T x), \omega_{\lambda}^{G}(T x, x, x), \omega_{\lambda}^{G}(x, T x, x), \omega_{\lambda}^{G}(x, x, T x), \\
\omega_{\lambda}^{G}(x, T x, T x), \omega_{\lambda}^{G}(x, T x, T x), \frac{\omega_{\lambda}^{G}(x, x, T x)\left[1+\omega_{\lambda}^{G}(x, T x, x)\right]}{1+\omega_{\lambda}^{G}(x, x, x)}, \\
\omega_{\lambda}^{G}(x, T x, x), \omega_{\lambda}^{G}(x, T x, T x), \frac{\omega_{\lambda}^{G}(x, T x, x)\left[1+\omega_{\lambda}^{G}(x, T x, x)\right]}{1+\omega_{\lambda}^{G}(x, x, x)}, \\
\omega_{\lambda}^{G}(x, T x, T x), \frac{\omega_{\lambda}^{G}(x, T x, x)\left[1+\omega_{\lambda}^{G}(x, x, x)\right]}{1+\omega_{\lambda}^{G}(x, x, x)+\omega_{\lambda}^{G}(x, T x, x)}, \omega_{\lambda}^{G}\left(x, T^{2} x, T x\right), \\
\omega_{\lambda}^{G}(x, T x, T x), \frac{\omega_{\lambda}^{G}(x, T x, x)\left[1+\omega_{\lambda}^{G}(x, x, T x)\right]}{1+\omega_{\lambda}^{G}(T x, x, x)+\omega_{\lambda}^{G}(x, x, T x)}, \omega_{\lambda}^{G}(x, T x, T x), \\
\omega_{\lambda}^{G}(x, T x, T x), \omega_{\lambda}^{G}(x, T x, T x), \omega_{\lambda}^{G}\left(x, T^{2} x, T x\right), \\
\omega_{\lambda}^{G}\left(T x, T^{2} x, T x\right), \omega_{\lambda}^{G}\left(T x, T^{2} x, T x\right), \frac{\omega_{\lambda}^{G}(x, x, T x)\left[1+\omega_{\lambda}^{G}(x, x, T x)\right]}{1+\omega_{\lambda}^{G}(T x, x, x)+\omega_{\lambda}^{G}(x, x, T x)}, \\
\frac{\omega_{\lambda}^{G}\left(T^{2} x, T x, T x\right)\left[1+\omega_{\lambda}^{G}(T x, x, x)+\omega_{\lambda}^{G}(T x, x, T x)\right]}{1+\omega_{\lambda}^{G}(T x, x, T x)+\omega_{\lambda}^{G}\left(T^{2} x, T x, T x\right)}
\end{array}\right\} .
\end{aligned}
$$


Now, we assume that $K_{\lambda}(x, x, x) \geq 0$ and $x \neq y=z$. Let $x_{0} \in X_{\omega^{G}}$ be arbitrary. We generate the sequence of iteration of $T$ based on $x_{0} \in X_{\omega^{G}}$ as follows:

$$
\begin{aligned}
& T x_{0}=x_{1} \\
& T x_{1}=x_{2} \\
& \vdots \\
& T x_{n}=x_{n+1}
\end{aligned}
$$

for all $n \in \mathbb{N}$. If there exists some $n_{0} \in \mathbb{N}$ such that $x_{n_{0}+1}=x_{n_{0}}$ then $x_{0}$ is a fixed point of $T$. Now for all $n \in \mathbb{N}, x_{n+1} \neq x_{n}$ and $\lambda>0$, take $x=x_{n}$ and $y=x_{n+1}=z$, then we have $\omega_{\lambda}^{G}\left(x_{n+1}, x_{n+2}, x_{n+2}\right)=\omega_{\lambda}^{G}(T x, T y, T z)=\omega_{\lambda}^{G}\left(T x_{n}, T x_{n+1}, T x_{n+1}\right)$, so that inequality (3.1) becomes

$$
\omega_{\lambda}^{G}\left(x_{n+1}, x_{n+2}, x_{n+2}\right) \leq \rho K_{\lambda}\left(x_{n}, x_{n+1}, x_{n+1}\right) \quad \forall x_{n}, x_{n+1} \in X_{\omega^{G}}, \lambda>0
$$

where

$$
\begin{aligned}
& K_{\lambda}\left(x_{n}, x_{n+1}, x_{n+1}\right)
\end{aligned}
$$

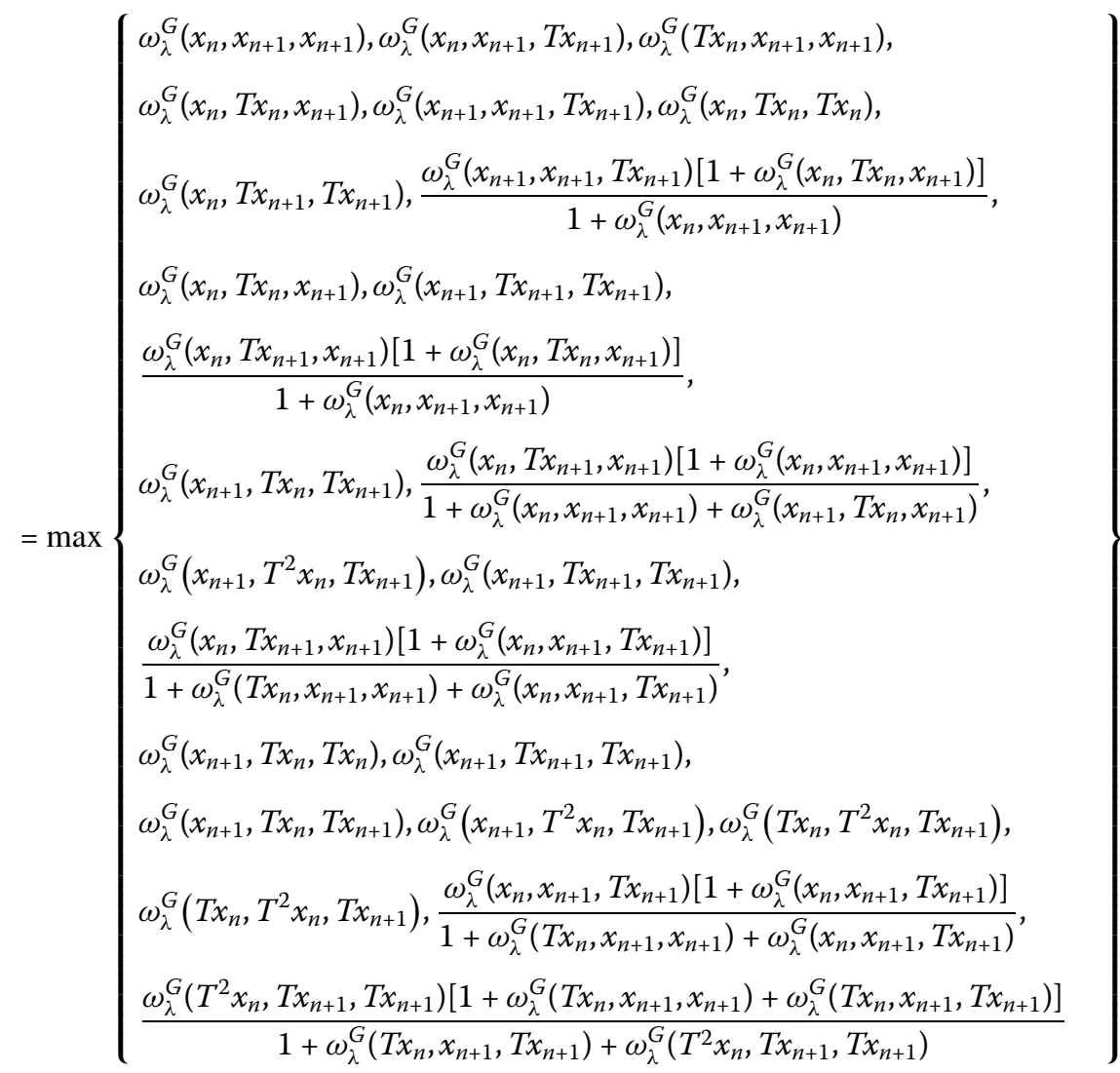




$$
=\max \left\{\begin{array}{l}
\omega_{\lambda}^{G}\left(x_{n}, x_{n+1}, x_{n+1}\right), \omega_{\lambda}^{G}\left(x_{n}, x_{n+1}, x_{n+2}\right), \omega_{\lambda}^{G}\left(x_{n+1}, x_{n+1}, x_{n+1}\right), \\
\omega_{\lambda}^{G}\left(x_{n}, x_{n+1}, x_{n+1}\right), \omega_{\lambda}^{G}\left(x_{n+1}, x_{n+1}, x_{n+2}\right), \omega_{\lambda}^{G}\left(x_{n}, x_{n+1}, x_{n+1}\right), \\
\omega_{\lambda}^{G}\left(x_{n}, x_{n+2}, x_{n+2}\right), \frac{\omega_{\lambda}^{G}\left(x_{n+1}, x_{n+1}, x_{n+2}\right)\left[1+\omega_{\lambda}^{G}\left(x_{n}, x_{n+1}, x_{n+1}\right)\right]}{1+\omega_{\lambda}^{G}\left(x_{n}, x_{n+1}, x_{n+1}\right)}, \\
\omega_{\lambda}^{G}\left(x_{n}, x_{n+1}, x_{n+1}\right), \omega_{\lambda}^{G}\left(x_{n+1}, x_{n+2}, x_{n+2}\right), \\
\frac{\omega_{\lambda}^{G}\left(x_{n}, x_{n+2}, x_{n+1}\right)\left[1+\omega_{\lambda}^{G}\left(x_{n}, x_{n+1}, x_{n+1}\right)\right]}{1+\omega_{\lambda}^{G}\left(x_{n}, x_{n+1}, x_{n+1}\right)}, \omega_{\lambda}^{G}\left(x_{n+1}, x_{n+1}, x_{n+2}\right), \\
\omega_{\lambda}^{G}\left(x_{n+1}, x_{n+2}, x_{n+2}\right), \frac{\omega_{\lambda}^{G}\left(x_{n}, x_{n+2}, x_{n+1}\right)\left[1+x_{n+1}\right)\left[1+\omega_{\lambda}^{G}\left(x_{n}, x_{n+1}, x_{n+1}\right)\right]}{1+\omega_{\lambda}^{G}\left(x_{n+1}, x_{n+1}, x_{n+1}\right)+\omega_{\lambda}^{G}\left(x_{n}, x_{n+1}, x_{n+2}\right)}, \omega_{\lambda}^{G}\left(x_{n+1}, x_{n+2}, x_{n+2}\right), \\
\left.1+\omega_{\lambda+2}^{G}\left(x_{n+1}, x_{n+1}, x_{n+1}\right)\right] \\
\omega_{\lambda}^{G}\left(x_{n+1}, x_{n+1}, x_{n+1}\right), \omega_{\lambda}^{G}\left(x_{n+1}, x_{n+2}, x_{n+2}\right), \omega_{\lambda}^{G}\left(x_{n+1}, x_{n+1}, x_{n+2}\right), \\
\omega_{\lambda}^{G}\left(x_{n+1}, x_{n+2}, x_{n+2}\right), \omega_{\lambda}^{G}\left(x_{n+1}, x_{n+2}, x_{n+2}\right), \omega_{\lambda}^{G}\left(x_{n+1}, x_{n+2}, x_{n+2}\right), \\
\frac{\omega_{\lambda}^{G}\left(x_{n}, x_{n+1}, x_{n+2}\right)\left[1+\omega_{\lambda}^{G}\left(x_{n}, x_{n+1}, x_{n+2}\right)\right]}{1+\omega_{\lambda}^{G}\left(x_{n+1}, x_{n+1}, x_{n+1}\right)+\omega_{\lambda}^{G}\left(x_{n}, x_{n+1}, x_{n+2}\right)},
\end{array}\right\} .
$$

On simplifying, we get

$$
\begin{aligned}
& K_{\lambda}\left(x_{n}, x_{n+1}, x_{n+1}\right)\left\{\begin{array}{l}
\omega_{\lambda}^{G}\left(x_{n}, x_{n+1}, x_{n+1}\right), \omega_{\lambda}^{G}\left(x_{n}, x_{n+1}, x_{n+2}\right), \omega_{\lambda}^{G}\left(x_{n+1}, x_{n+1}, x_{n+1}\right), \\
\omega_{\lambda}^{G}\left(x_{n}, x_{n+1}, x_{n+1}\right), \omega_{\lambda}^{G}\left(x_{n+1}, x_{n+1}, x_{n+2}\right), \omega_{\lambda}^{G}\left(x_{n}, x_{n+1}, x_{n+1}\right), \\
\omega_{\lambda}^{G}\left(x_{n}, x_{n+2}, x_{n+2}\right), \omega_{\lambda}^{G}\left(x_{n+1}, x_{n+1}, x_{n+2}\right), \omega_{\lambda}^{G}\left(x_{n}, x_{n+1}, x_{n+1}\right), \\
\omega_{\lambda}^{G}\left(x_{n+1}, x_{n+2}, x_{n+2}\right), \omega_{\lambda}^{G}\left(x_{n}, x_{n+2}, x_{n+1}\right), \omega_{\lambda}^{G}\left(x_{n+1}, x_{n+1}, x_{n+2}\right), \\
\omega_{\lambda}^{G}\left(x_{n}, x_{n+2}, x_{n+1}\right), \omega_{\lambda}^{G}\left(x_{n+1}, x_{n+2}, x_{n+2}\right), \omega_{\lambda}^{G}\left(x_{n+1}, x_{n+2}, x_{n+2}\right), \\
\omega_{\lambda}^{G}\left(x_{n}, x_{n+2}, x_{n+1}\right), \omega_{\lambda}^{G}\left(x_{n+1}, x_{n+1}, x_{n+1}\right), \omega_{\lambda}^{G}\left(x_{n+1}, x_{n+2}, x_{n+2}\right), \\
\omega_{\lambda}^{G}\left(x_{n+1}, x_{n+1}, x_{n+2}\right), \omega_{\lambda}^{G}\left(x_{n+1}, x_{n+2}, x_{n+2}\right), \omega_{\lambda}^{G}\left(x_{n+1}, x_{n+2}, x_{n+2}\right), \\
\omega_{\lambda}^{G}\left(x_{n+1}, x_{n+2}, x_{n+2}\right), \omega_{\lambda}^{G}\left(x_{n}, x_{n+1}, x_{n+2}\right), \omega_{\lambda}^{G}\left(x_{n+2}, x_{n+2}, x_{n+2}\right)
\end{array}\right\} \\
&=\max \left\{\begin{array}{l}
\omega_{\lambda}^{G}\left(x_{n}, x_{n+1}, x_{n+1}\right), \omega_{\lambda}^{G}\left(x_{n}, x_{n+1}, x_{n+2}\right), \\
\left.\omega_{\lambda}^{G}\left(x_{n}, x_{n+2}, x_{n+2}\right), \omega_{\lambda}^{G}\left(x_{n+1}, x_{n+1}, x_{n+2}\right),\right\} . \\
\omega_{\lambda}^{G}\left(x_{n+1}, x_{n+2}, x_{n+2}\right)
\end{array}\right\}
\end{aligned}
$$

We will examine this in five different cases as follows.

Case 1. If $K_{\lambda}\left(x_{n}, x_{n+1}, x_{n+1}\right)=\omega_{\lambda}^{G}\left(x_{n}, x_{n+1}, x_{n+1}\right)$, then

$$
\omega_{\lambda}^{G}\left(x_{n+1}, x_{n+2}, x_{n+2}\right) \leq \rho \omega_{\lambda}^{G}\left(x_{n}, x_{n+1}, x_{n+1}\right),
$$

for $\rho \in(0,1)$. 
Case 2. If $K_{\lambda}\left(x_{n}, x_{n+1}, x_{n+1}\right)=\omega_{\lambda}^{G}\left(x_{n}, x_{n+1}, x_{n+2}\right)$, then using conditions (1), (4) of Definition 2.1, conditions (3), (6) of Proposition 2.2 and Definition 2.11 twice, we get

$$
\begin{aligned}
\omega_{\lambda}^{G}\left(x_{n+1}, x_{n+2}, x_{n+2}\right) & \leq \rho \omega_{\lambda}^{G}\left(x_{n}, x_{n+1}, x_{n+2}\right) \\
& \leq \rho\left[\omega_{\frac{\lambda}{2}}^{G}\left(x_{n}, x_{n+1}, x_{n+1}\right)+\omega_{\frac{\lambda}{4}}^{G}\left(x_{n+1}, x_{n+1}, x_{n+1}\right)+\omega_{\frac{\lambda}{4}}^{G}\left(x_{n+1}, x_{n+1}, x_{n+2}\right)\right] \\
& =\rho\left[\omega_{\frac{\lambda}{2}}^{G}\left(x_{n}, x_{n+1}, x_{n+1}\right)+\omega_{\frac{\lambda}{4}}^{G}\left(x_{n+1}, x_{n+1}, x_{n+2}\right)\right] \\
& \leq \rho\left(C_{2} \omega_{\lambda}^{G}\left(x_{n}, x_{n+1}, x_{n+1}\right)+C_{4} \omega_{\lambda}^{G}\left(x_{n+1}, x_{n+1}, x_{n+2}\right)\right) \\
& \leq \rho\left(C_{2} \omega_{\lambda}^{G}\left(x_{n}, x_{n+1}, x_{n+1}\right)+2 C_{4} \omega_{\frac{\lambda}{2}}^{G}\left(x_{n+1}, x_{n+2}, x_{n+2}\right)\right) \\
& \leq \rho\left(C_{2} \omega_{\lambda}^{G}\left(x_{n}, x_{n+1}, x_{n+1}\right)+2 C_{2} C_{4} \omega_{\lambda}^{G}\left(x_{n+1}, x_{n+2}, x_{n+2}\right)\right),
\end{aligned}
$$

which implies that

$$
\omega_{\lambda}^{G}\left(x_{n+1}, x_{n+2}, x_{n+2}\right) \leq \rho\left(C_{2} \omega_{\lambda}^{G}\left(x_{n}, x_{n+1}, x_{n+1}\right)+2 C_{2} C_{4} \omega_{\lambda}^{G}\left(x_{n+1}, x_{n+2}, x_{n+2}\right)\right),
$$

hence, we have

$$
\begin{aligned}
& \omega_{\lambda}^{G}\left(x_{n+1}, x_{n+2}, x_{n+2}\right) \leq \frac{\rho C_{2}}{1-2 \rho C_{2} C_{4}} \omega_{\lambda}^{G}\left(x_{n}, x_{n+1}, x_{n+1}\right), \\
& \omega_{\lambda}^{G}\left(x_{n+1}, x_{n+2}, x_{n+2}\right) \leq k_{j} \omega_{\lambda}^{G}\left(x_{n}, x_{n+1}, x_{n+1}\right),
\end{aligned}
$$

where $k_{j}=\frac{\rho C_{2}}{1-j \rho C_{2} C_{4}}<1$, so that $C_{2} C_{4} \in\left(0, \frac{1}{j \rho}\right)$, where $j=2$.

Case 3. If $K_{\lambda}\left(x_{n}, x_{n+1}, x_{n+1}\right)=\omega_{\lambda}^{G}\left(x_{n}, x_{n+2}, x_{n+2}\right)$, then using conditions (3), (6) of Proposition 2.2 and Definition 2.11, we have

$$
\begin{aligned}
\omega_{\lambda}^{G}\left(x_{n+1}, x_{n+2}, x_{n+2}\right) & \leq \rho \omega_{\lambda}^{G}\left(x_{n}, x_{n+2}, x_{n+2}\right) \\
& \leq \rho\left[\omega_{\frac{\lambda}{2}}^{G}\left(x_{n}, x_{n+1}, x_{n+1}\right)+\omega_{\frac{\lambda}{4}}^{G}\left(x_{n+2}, x_{n+1}, x_{n+1}\right)+\omega_{\frac{\lambda}{4}}^{G}\left(x_{n+2}, x_{n+1}, x_{n+1}\right)\right] \\
& =\rho\left(\omega_{\frac{\lambda}{2}}^{G}\left(x_{n}, x_{n+1}, x_{n+1}\right)+2 \omega_{\frac{\lambda}{4}}^{G}\left(x_{n+1}, x_{n+1}, x_{n+2}\right)\right) \\
& \leq \rho\left(C_{2} \omega_{\lambda}^{G}\left(x_{n}, x_{n+1}, x_{n+1}\right)+2 C_{4} \omega_{\lambda}^{G}\left(x_{n+1}, x_{n+1}, x_{n+2}\right)\right) \\
& \leq \rho\left(C_{2} \omega_{\lambda}^{G}\left(x_{n}, x_{n+1}, x_{n+1}\right)+4 C_{4} \omega_{\frac{\lambda}{2}}^{G}\left(x_{n+1}, x_{n+2}, x_{n+2}\right)\right) \\
& \leq \rho\left(C_{2} \omega_{\lambda}^{G}\left(x_{n}, x_{n+1}, x_{n+1}\right)+4 C_{2} C_{4} \omega_{\lambda}^{G}\left(x_{n+1}, x_{n+2}, x_{n+2}\right)\right)
\end{aligned}
$$

which implies that

$$
\omega_{\lambda}^{G}\left(x_{n+1}, x_{n+2}, x_{n+2}\right) \leq \frac{\rho C_{2}}{1-4 \rho C_{2} C_{4}} \omega_{\lambda}^{G}\left(x_{n}, x_{n+1}, x_{n+1}\right),
$$

hence, we have

$$
\omega_{\lambda}^{G}\left(x_{n+1}, x_{n+2}, x_{n+2}\right) \leq k_{j} \omega_{\lambda}^{G}\left(x_{n}, x_{n+1}, x_{n+1}\right)
$$

where $k_{j}=\frac{\rho C_{2}}{1-j \rho C_{2} C_{4}}<1$, so that $C_{2} C_{4} \in\left(0, \frac{1}{j \rho}\right)$, where $j=4$. 
Case 4. If $K_{\lambda}\left(x_{n}, x_{n+1}, x_{n+1}\right)=\omega_{\lambda}^{G}\left(x_{n+1}, x_{n+1}, x_{n+2}\right)$, then using condition (6) of Proposition 2.2 and Definition 2.11, inequality (3.3) becomes

$$
\begin{aligned}
& \omega_{\lambda}^{G}\left(x_{n+1}, x_{n+2}, x_{n+2}\right) \\
& \leq \rho\left(\omega_{\frac{\lambda}{2}}^{G}\left(x_{n}, x_{n}, x_{n+1}\right)+\omega_{\frac{\lambda}{4}}^{G}\left(x_{n}, x_{n}, x_{n+1}\right)+\omega_{\frac{\lambda}{4}}^{G}\left(x_{n}, x_{n}, x_{n+2}\right)\right) \\
& \leq \rho\left(C_{2} \omega_{\lambda}^{G}\left(x_{n+1}, x_{n}, x_{n}\right)+C_{4} \omega_{\lambda}^{G}\left(x_{n+1}, x_{n}, x_{n}\right)+C_{4} \omega_{\lambda}^{G}\left(x_{n+2}, x_{n}, x_{n}\right)\right) \\
& =\rho\left(\left(C_{4}+C_{2}\right) \omega_{\lambda}^{G}\left(x_{n+1}, x_{n}, x_{n}\right)+C_{4} \omega_{\lambda}^{G}\left(x_{n+2}, x_{n}, x_{n}\right)\right) \\
& \leq \rho\left(( C _ { 4 } + C _ { 2 } ) \left[\omega_{\frac{\lambda}{2}}^{G}\left(x_{n+1}, x_{n+2}, x_{n+2}\right)+\omega_{\frac{\lambda}{4}}^{G}\left(x_{n}, x_{n+2}, x_{n+2}\right)\right.\right. \\
& \left.\left.+\omega_{\lambda}^{G}\left(x_{n}, x_{n+2}, x_{n+2}\right)\right]+C_{4} \omega_{\lambda}^{G}\left(x_{n+2}, x_{n}, x_{n}\right)\right) \\
& \leq \rho\left(\left(C_{2}+C_{4}\right)\left(C_{2} \omega_{\lambda}^{G}\left(x_{n+1}, x_{n+2}, x_{n+2}\right)+2 C_{4} \omega_{\lambda}^{G}\left(x_{n}, x_{n+2}, x_{n+2}\right)\right)\right. \\
& \left.+C_{4} \omega_{\lambda}^{G}\left(x_{n+2}, x_{n}, x_{n}\right)\right) \\
& \leq \rho\left(( C _ { 2 } + C _ { 4 } ) \left(C_{2} \omega_{\lambda}^{G}\left(x_{n+1}, x_{n+2}, x_{n+2}\right)+2 C_{4}\left(\omega_{\frac{\lambda}{2}}^{G}\left(x_{n}, x_{n}, x_{n+2}\right)\right.\right.\right. \\
& \left.\left.\left.+\omega_{\frac{\lambda}{2}}^{G}\left(x_{n}, x_{n}, x_{n+2}\right)\right)\right)+C_{4} \omega_{\lambda}^{G}\left(x_{n+2}, x_{n}, x_{n}\right)\right) \\
& =\rho\left(\left(C_{2}+C_{4}\right)\left(C_{2} \omega_{\lambda}^{G}\left(x_{n+1}, x_{n+2}, x_{n+2}\right)+2 C_{4}\left(2 \omega_{\frac{\lambda}{2}}^{G}\left(x_{n}, x_{n}, x_{n+2}\right)\right)\right)\right. \\
& \left.+C_{4} \omega_{\lambda}^{G}\left(x_{n+2}, x_{n}, x_{n}\right)\right) \\
& \leq \rho\left(\left(C_{2}+C_{4}\right)\left(C_{2} \omega_{\lambda}^{G}\left(x_{n+1}, x_{n+2}, x_{n+2}\right)+2 C_{4}\left(2 C_{2} \omega_{\lambda}^{G}\left(x_{n}, x_{n}, x_{n+2}\right)\right)\right)\right. \\
& \left.+C_{4} \omega_{\lambda}^{G}\left(x_{n+2}, x_{n}, x_{n}\right)\right) \\
& =\rho\left(\left(C_{2}+C_{4}\right)\left(C_{2} \omega_{\lambda}^{G}\left(x_{n+1}, x_{n+2}, x_{n+2}\right)+4 C_{4} C_{2} \omega_{\lambda}^{G}\left(x_{n}, x_{n}, x_{n+2}\right)\right)\right. \\
& \left.+C_{4} \omega_{\lambda}^{G}\left(x_{n+2}, x_{n}, x_{n}\right)\right) \\
& =\rho\left(C_{2}\left(C_{2}+C_{4}\right) \omega_{\lambda}^{G}\left(x_{n+1}, x_{n+2}, x_{n+2}\right)+C_{4}\left(1+4 C_{2}\left(C_{2}+C_{4}\right)\right) \omega_{\lambda}^{G}\left(x_{n}, x_{n}, x_{n+2}\right)\right) \\
& \leq \rho\left(C_{2}\left(C_{2}+C_{4}\right) \omega_{\lambda}^{G}\left(x_{n+1}, x_{n+2}, x_{n+2}\right)+C_{4}\left(1+4 C_{2}\left(C_{2}+C_{4}\right)\right)\right. \\
& \left.\times\left[\omega_{\frac{\lambda}{2}}^{G}\left(x_{n}, x_{n+1}, x_{n+1}\right)+\omega_{\frac{\lambda}{4}}^{G}\left(x_{n}, x_{n+1}, x_{n+1}\right)+\omega_{\frac{\lambda}{4}}^{G}\left(x_{n+1}, x_{n+1}, x_{n+2}\right)\right]\right) \\
& \leq \rho\left(C_{2}\left(C_{2}+C_{4}\right) \omega_{\lambda}^{G}\left(x_{n+1}, x_{n+2}, x_{n+2}\right)+C_{4}\left(1+4 C_{2}\left(C_{2}+C_{4}\right)\right)\right. \\
& \left.\times\left[C_{2} \omega_{\lambda}^{G}\left(x_{n}, x_{n+1}, x_{n+1}\right)+C_{4} \omega_{\lambda}^{G}\left(x_{n}, x_{n+1}, x_{n+1}\right)+C_{4} \omega_{\lambda}^{G}\left(x_{n+1}, x_{n+1}, x_{n+2}\right)\right]\right) \\
& =\rho\left(C_{2}\left(C_{2}+C_{4}\right) \omega_{\lambda}^{G}\left(x_{n+1}, x_{n+2}, x_{n+2}\right)+C_{4}\left(1+4 C_{2}\left(C_{2}+C_{4}\right)\right)\right. \\
& \left.\times\left[\left(C_{2}+C_{4}\right) \omega_{\lambda}^{G}\left(x_{n}, x_{n+1}, x_{n+1}\right)+C_{4} \omega_{\lambda}^{G}\left(x_{n+1}, x_{n+1}, x_{n+2}\right)\right]\right) \\
& \leq \rho\left(C_{2}\left(C_{2}+C_{4}\right) \omega_{\lambda}^{G}\left(x_{n+1}, x_{n+2}, x_{n+2}\right)+C_{4}\left(1+4 C_{2}\left(C_{2}+C_{4}\right)\right)\right. \\
& \left.\times\left[\left(C_{2}+C_{4}\right) \omega_{\lambda}^{G}\left(x_{n}, x_{n+1}, x_{n+1}\right)+2 C_{4} \omega_{\frac{\lambda}{2}}^{G}\left(x_{n+1}, x_{n+2}, x_{n+2}\right)\right]\right) \\
& \leq \rho\left(C_{2}\left(C_{2}+C_{4}\right) \omega_{\lambda}^{G}\left(x_{n+1}, x_{n+2}, x_{n+2}\right)+C_{4}\left(1+4 C_{2}\left(C_{2}+C_{4}\right)\right)\right. \\
& \left.\times\left[\left(C_{2}+C_{4}\right) \omega_{\lambda}^{G}\left(x_{n}, x_{n+1}, x_{n+1}\right)+2 C_{4} C_{2} \omega_{\lambda}^{G}\left(x_{n+1}, x_{n+2}, x_{n+2}\right)\right]\right) \\
& =\rho\left(C_{2}\left(C_{2}+C_{4}\right)+2 C_{4}^{2} C_{2}\left(1+4 C_{2}\left(C_{2}+C_{4}\right)\right) \omega_{\lambda}^{G}\left(x_{n+1}, x_{n+2}, x_{n+2}\right)\right. \\
& \left.+C_{4}\left(C_{2}+C_{4}\right)\left(1+4 C_{2}\left(C_{2}+C_{4}\right)\right) \omega_{\lambda}^{G}\left(x_{n}, x_{n+1}, x_{n+1}\right)\right) .
\end{aligned}
$$


Therefore, it follows from inequality (3.7) that

$$
\omega_{\lambda}^{G}\left(x_{n+1}, x_{n+2}, x_{n+2}\right) \leq k_{3} \omega_{\lambda}^{G}\left(x_{n}, x_{n+1}, x_{n+1}\right)
$$

where $k_{3}:=\frac{\rho C_{4}\left(C_{2}+C_{4}\right)\left(1+4 C_{2}\left(C_{2}+C_{4}\right)\right)}{1-j \rho\left(C_{2}\left(C_{2}+C_{4}\right)+2 C_{4}^{2} C_{2}\left(1+4 C_{2}\left(C_{2}+C_{4}\right)\right)\right)}<1$ and $\left(C_{2}\left(C_{2}+C_{4}\right)+2 C_{4}^{2} C_{2}\left(1+4 C_{2}\left(C_{2}+\right.\right.\right.$ $\left.\left.\left.C_{4}\right)\right)\right) \in\left(0, \frac{1}{j \rho}\right)$ for $j=1$.

Case 5. If $K_{\lambda}\left(x_{n}, x_{n+1}, x_{n+1}\right)=\omega_{\lambda}^{G}\left(x_{n+1}, x_{n+2}, x_{n+2}\right)$, then inequality (3.3) becomes

$$
\omega_{\lambda}^{G}\left(x_{n+1}, x_{n+2}, x_{n+2}\right) \leq \rho \omega_{\lambda}^{G}\left(x_{n+1}, x_{n+2}, x_{n+2}\right)
$$

and $\rho \in(0,1)$.

Now, we take $h=\max \left\{\rho, k_{j}, k_{3}\right\}$, for $j=1,2,4, \ldots$, so that

$$
\begin{aligned}
\omega_{\lambda}^{G}\left(x_{n+1}, x_{n+2}, x_{n+2}\right) & \leq h \omega_{\lambda}^{G}\left(x_{n}, x_{n+1}, x_{n+1}\right) \\
\leq & h^{2} \omega_{\lambda}^{G}\left(x_{n-1}, x_{n}, x_{n}\right) \\
\leq & h^{3} \omega_{\lambda}^{G}\left(x_{n-2}, x_{n-1}, x_{n-1}\right) \\
& \vdots \\
\leq & \leq h^{n} \omega_{\lambda}^{G}\left(x_{0}, x_{1}, x_{1}\right) .
\end{aligned}
$$

But $\sum_{n \in \mathbb{N}} h^{n}<+\infty$. Now $\sum_{n \in \mathbb{N}} \omega_{\lambda}^{G}\left(x_{n+1}, x_{n+2}, x_{n+2}\right) \leq \omega_{\lambda}^{G}\left(x_{0}, x_{1}, x_{1}\right) \sum_{n \in \mathbb{N}} h^{n}<+\infty$ for all $\lambda>0$. Suppose that $m, n \in \mathbb{N}$ and $m>n \in \mathbb{N}$. Observe that, for any arbitrary $\epsilon$, using the rectangle inequality repeatedly and condition (2) of Proposition 2.2, we have

$$
\begin{aligned}
\omega_{\lambda}^{G}\left(x_{n}, x_{m}, x_{m}\right) \leq & \omega_{\frac{\lambda}{m-n}}^{G}\left(x_{n}, x_{n+1}, x_{n+1}\right)+\omega_{\frac{\lambda}{m-n}}^{G}\left(x_{n+1}, x_{n+2}, x_{n+2}\right)+\omega_{\frac{\lambda}{m-n}}^{G}\left(x_{n+2}, x_{n+3}, x_{n+3}\right) \\
& +\omega_{\frac{\lambda}{m-n}}^{G}\left(x_{n+3}, x_{n+4}, x_{n+4}\right)+\cdots+\omega_{\frac{\lambda}{m-n}}^{G}\left(x_{m-1}, x_{m}, x_{m}\right) \\
\leq & \omega_{\frac{\lambda}{m}}^{G}\left(x_{n}, x_{n+1}, x_{n+1}\right)+\omega_{\frac{\lambda}{m}}^{G}\left(x_{n+1}, x_{n+2}, x_{n+2}\right)+\omega_{\frac{\lambda}{m}}^{G}\left(x_{n+2}, x_{n+3}, x_{n+3}\right) \\
& +\omega_{\frac{\lambda}{m}}^{G}\left(x_{n+3}, x_{n+4}, x_{n+4}\right)+\cdots+\omega_{\frac{\lambda}{m}}^{G}\left(x_{m-1}, x_{m}, x_{m}\right) \\
\leq & \sum_{n=N} \omega_{\lambda}^{G}\left(x_{n+1}, x_{n+2}, x_{n+2}\right) \\
< & \epsilon
\end{aligned}
$$

for all $m>n \geq N$ for some $N \in \mathbb{N}$. As $\epsilon$ is arbitrary, we have

$$
\omega_{\lambda}^{G}\left(x_{n}, x_{m}, x_{m}\right)=0 \quad \text { as } n, m \rightarrow \infty \quad \text { or } \quad \lim _{n, m \rightarrow \infty} \omega_{\lambda}^{G}\left(x_{n}, x_{m}, x_{m}\right)=0
$$

For $n, m, k \in \mathbb{N}$, condition (2) of Proposition 2.2 implies that

$$
\omega_{\lambda}^{G}\left(x_{n}, x_{m}, x_{k}\right) \leq \omega_{\frac{\lambda}{2}}^{G}\left(x_{n}, x_{m}, x_{m}\right)+\omega_{\frac{\lambda}{2}}^{G}\left(x_{k}, x_{m}, x_{m}\right)
$$


so that on taking the limit of both sides of inequality (3.13) as $n, m, l \rightarrow \infty$ and by applying Definition 2.11 and Eq. (3.12), we get

$$
\begin{aligned}
\lim _{n, m, k \rightarrow \infty} \omega_{\lambda}^{G}\left(x_{n}, x_{m}, x_{k}\right) & \leq \lim _{n, m \rightarrow \infty} \omega_{\lambda}^{G}\left(x_{n}, x_{m}, x_{m}\right)+\lim _{k, m \rightarrow \infty} \omega_{\frac{\lambda}{2}}^{G}\left(x_{k}, x_{m}, x_{m}\right) \\
& \leq C_{2} \lim _{n, m \rightarrow \infty} \omega_{\lambda}^{G}\left(x_{n}, x_{m}, x_{m}\right)+C_{2} \lim _{k, m \rightarrow \infty} \omega_{\lambda}^{G}\left(x_{k}, x_{m}, x_{m}\right) \\
& =C_{2}\left(\lim _{n, m \rightarrow \infty} \omega_{\lambda}^{G}\left(x_{n}, x_{m}, x_{m}\right)+\lim _{k, m \rightarrow \infty} \omega_{\lambda}^{G}\left(x_{k}, x_{m}, x_{m}\right)\right) ;
\end{aligned}
$$

thus we have

$$
\lim _{n, m, k \rightarrow \infty} \omega_{\lambda}^{G}\left(x_{n}, x_{m}, x_{k}\right)=0 \quad \forall \lambda>0 .
$$

Equation (3.15) confirmed that the sequence $\left\{x_{n}\right\}_{n \in \mathbb{N}}$ is modular $G$-Cauchy sequence. The completeness of $\left(X, \omega^{G}\right)$ implies that, for any $\lambda>0, \lim _{n, m \rightarrow \infty} \omega_{\lambda}^{G}\left(x_{n}, x_{m}, u\right)=0$, i.e. for any $\epsilon>0$, there exists $n_{0} \in \mathbb{N}$ such that $\omega_{\lambda}^{G}\left(x_{n}, x_{m}, u\right)<\epsilon$ for all $n, m \in \mathbb{N}$ and $n, m \geq n_{0}$, which implies that $\lim _{n \rightarrow \infty} x_{n} \rightarrow u$. Suppose, if possible, that $T u \neq u$, i.e. $\omega_{\lambda}^{G}(u, T u, T u)>0$, then from inequality (3.1), with $x=x_{n}, y=u=z$, we have

$$
\omega_{\lambda}^{G}\left(x_{n+1}, T u, T u\right)=\omega_{\lambda}^{G}\left(T x_{n}, T u, T u\right) \leq \rho K_{\lambda}\left(x_{n}, u, u\right) \quad \text { for all } x_{n}, u, \in X_{\omega^{G}}, \lambda>0,
$$

so that

$$
\begin{aligned}
& K_{\lambda}\left(x_{n}, u, u\right) \\
& =\max \left\{\begin{array}{l}
\omega_{\lambda}^{G}\left(x_{n}, u, u\right), \omega_{\lambda}^{G}\left(x_{n}, u, T u\right), \omega_{\lambda}^{G}\left(T x_{n}, u, u\right), \omega_{\lambda}^{G}\left(x_{n}, T x_{n}, u\right), \\
\omega_{\lambda}^{G}(u, u, T u), \omega_{\lambda}^{G}\left(x_{n}, T x_{n}, T x_{n}\right), \omega_{\lambda}^{G}\left(x_{n}, T u, T u\right), \\
\frac{\omega_{\lambda}^{G}(u, u, T u)\left[1+\omega_{\lambda}^{G}\left(x_{n}, T x_{n}, u\right)\right]}{1+\omega_{\lambda}^{G}\left(x_{n}, u, u\right)}, \omega_{\lambda}^{G}\left(x_{n}, T x_{n}, u\right), \omega_{\lambda}^{G}(u, T u, T u), \\
\frac{\omega_{\lambda}^{G}\left(x_{n}, T u, u\right)\left[1+\omega_{\lambda}^{G}\left(x_{n}, T x_{n}, u\right)\right]}{1+\omega_{\lambda}^{G}\left(x_{n}, u, u\right)}, \omega_{\lambda}^{G}\left(u, T x_{n}, T u\right), \\
1+\omega_{\lambda}^{G}\left(x_{n}, u, u\right)+\omega_{\lambda}^{G}\left(u, T x_{n}, u\right) \\
\omega_{\lambda}^{G}(u, T u, T u), \frac{\omega_{\lambda}^{G}\left(x_{n}, T u, u\right)\left[1+\omega_{\lambda}^{G}\left(x_{n}, u, T u\right)\right]}{1+\omega_{\lambda}^{G}\left(T x_{n}, u, u\right)+\omega_{\lambda}^{G}\left(x_{n}, u, T u\right)}, \omega_{\lambda}^{G}\left(u, T x_{n}, T x_{n}\right), \\
\omega_{\lambda}^{G}(u, T u, T u), \omega_{\lambda}^{G}\left(u, T x_{n}, T u\right), \omega_{\lambda}^{G}\left(u, T^{2} x_{n}, T u\right), \omega_{\lambda}^{G}\left(T x_{n}, T^{2} x_{n}, T u\right), \\
\omega_{\lambda}^{G}\left(T x_{n}, T^{2} x_{n}, T u\right), \frac{\omega_{\lambda}^{G}\left(x_{n}, u, T u\right)\left[1+\omega_{\lambda}^{G}\left(x_{n}, u, T u\right)\right]}{1+\omega_{\lambda}^{G}\left(T x_{n}, u, u\right)+\omega_{\lambda}^{G}\left(x_{n}, u, T u\right)}, \\
\frac{\omega_{\lambda}^{G}\left(T^{2} x_{n}, T u, T u\right)\left[1+\omega_{\lambda}^{G}\left(T x_{n}, u, u\right)+\omega_{\lambda}^{G}\left(T x_{n}, u, T u\right)\right]}{1+\omega_{\lambda}^{G}\left(T x_{n}, u, T u\right)+\omega_{\lambda}^{G}\left(T^{2} x_{n}, T u, T u\right)}
\end{array}\right\} .
\end{aligned}
$$


Thus

$$
\begin{aligned}
& K_{\lambda}\left(x_{n}, u, u\right) \\
& =\max \left\{\begin{array}{l}
\omega_{\lambda}^{G}\left(x_{n}, u, u\right), \omega_{\lambda}^{G}\left(x_{n}, u, T u\right), \omega_{\lambda}^{G}\left(x_{n+1}, u, u\right), \omega_{\lambda}^{G}\left(x_{n}, x_{n+1}, u\right), \\
\omega_{\lambda}^{G}(u, u, T u), \omega_{\lambda}^{G}\left(x_{n}, x_{n+1}, x_{n+1}\right), \omega_{\lambda}^{G}\left(x_{n}, T u, T u\right), \\
\frac{\omega_{\lambda}^{G}(u, u, T u)\left[1+\omega_{\lambda}^{G}\left(x_{n}, x_{n+1}, u\right)\right]}{1+\omega_{\lambda}^{G}\left(x_{n}, u, u\right)}, \omega_{\lambda}^{G}\left(x_{n}, x_{n+1}, u\right), \omega_{\lambda}^{G}(u, T u, T u), \\
\frac{\omega_{\lambda}^{G}\left(x_{n}, T u, u\right)\left[1+\omega_{\lambda}^{G}\left(x_{n}, x_{n+1}, u\right)\right]}{1+\omega_{\lambda}^{G}\left(x_{n}, u, u\right)}, \omega_{\lambda}^{G}\left(u, x_{n+1}, T u\right), \\
\frac{\omega_{\lambda}^{G}\left(x_{n}, T u, u\right)\left[1+\omega_{\lambda}^{G}\left(x_{n}, u, T u\right)\right]}{1+\omega_{\lambda}^{G}\left(x_{n+1}, u, u\right)+\omega_{\lambda}^{G}\left(x_{n}, u, T u\right)}, \omega_{\lambda}^{G}\left(u, x_{n+1}, x_{n+1}\right), \\
\left.\omega_{\lambda}^{G}(u, T u, T u), \omega_{\lambda}^{G}\left(u, x_{n+1}, T u\right), \omega_{\lambda}^{G}\left(u, x_{n+2}, T u\right), \omega_{\lambda}^{G}\left(x_{n+1}, x_{n+2}, T u\right), u, x_{n+1}, u\right) \\
1, \omega_{\lambda}^{G}\left(u, x_{n+2}, T u\right), \omega_{\lambda}^{G}(u, T u, T u), \\
\omega_{\lambda}^{G}\left(x_{n+1}, x_{n+2}, T u\right), \frac{\omega_{\lambda}^{G}\left(x_{n}, u, T u\right)\left[1+\omega_{\lambda}^{G}\left(x_{n}, u, T u\right)\right]}{1+\omega_{\lambda}^{G}\left(x_{n+1}, u, u\right)+\omega_{\lambda}^{G}\left(x_{n}, u, T u\right)} \\
\frac{\omega_{\lambda}^{G}\left(x_{n+2}, T u, T u\right)\left[1+\omega_{\lambda}^{G}\left(x_{n+1}, u, u\right)+\omega_{\lambda}^{G}\left(x_{n+1}, u, T u\right)\right]}{1+\omega_{\lambda}^{G}\left(x_{n+1}, u, T u\right)+\omega_{\lambda}^{G}\left(x_{n+2}, T u, T u\right)}
\end{array}\right\} .
\end{aligned}
$$

Since $\lim _{n \rightarrow \infty} x_{n} \rightarrow u \in X_{\omega}$,

$K_{\lambda}(u, u, u)$

$$
=\max \left\{\begin{array}{l}
\omega_{\lambda}^{G}(u, u, u), \omega_{\lambda}^{G}(u, u, T u), \omega_{\lambda}^{G}(u, u, u), \omega_{\lambda}^{G}(u, u, u), \\
\omega_{\lambda}^{G}(u, u, T u), \omega_{\lambda}^{G}(u, u, u), \omega_{\lambda}^{G}(u, T u, T u), \frac{\omega_{\lambda}^{G}(u, u, T u)\left[1+\omega_{\lambda}^{G}(u, u, u)\right]}{1+\omega_{\lambda}^{G}(u, u, u)}, \\
\omega_{\lambda}^{G}(u, u, u), \omega_{\lambda}^{G}(u, T u, T u), \frac{\omega_{\lambda}^{G}(u, T u, u)\left[1+\omega_{\lambda}^{G}(u, u, u)\right]}{1+\omega_{\lambda}^{G}(u, u, u)}, \\
\omega_{\lambda}^{G}(u, u, T u), \frac{\omega_{\lambda}^{G}(u, T u, u)\left[1+\omega_{\lambda}^{G}(u, u, u)\right]}{1+\omega_{\lambda}^{G}(u, u, u)+\omega_{\lambda}^{G}(u, u, u)}, \omega_{\lambda}^{G}(u, u, T u), \\
\omega_{\lambda}^{G}(u, T u, T u), \frac{\omega_{\lambda}^{G}(u, T u, u)\left[1+\omega_{\lambda}^{G}(u, u, T u)\right]}{1+\omega_{\lambda}^{G}(u, u, u)+\omega_{\lambda}^{G}(u, u, T u)}, \omega_{\lambda}^{G}(u, u, u), \\
\omega_{\lambda}^{G}(u, T u, T u), \omega_{\lambda}^{G}(u, u, T u), \omega_{\lambda}^{G}(u, u, T u), \omega_{\lambda}^{G}(u, u, T u), \omega_{\lambda}^{G}(u, u, T u), \\
\frac{\omega_{\lambda}^{G}(u, u, T u)\left[1+\omega_{\lambda}^{G}(u, u, T u)\right]}{1+\omega_{\lambda}^{G}(u, u, u)+\omega_{\lambda}^{G}(u, u, T u)} \\
\frac{\omega_{\lambda}^{G}(u, T u, T u)\left[1+\omega_{\lambda}^{G}(u, u, u)+\omega_{\lambda}^{G}(u, u, T u)\right]}{1+\omega_{\lambda}^{G}(u, u, T u)+\omega_{\lambda}^{G}(u, T u, T u)}
\end{array}\right\},
$$


which gives

$$
K_{\lambda}(u, u, u)=\max \left\{\begin{array}{l}
\omega_{\lambda}^{G}(u, u, u), \omega_{\lambda}^{G}(u, u, T u), \\
\omega_{\lambda}^{G}(u, u, u), \omega_{\lambda}^{G}(u, u, u), \\
\omega_{\lambda}^{G}(u, u, T u), \omega_{\lambda}^{G}(u, u, u), \\
\omega_{\lambda}^{G}(u, T u, T u), \omega_{\lambda}^{G}(u, u, T u), \\
\omega_{\lambda}^{G}(u, u, u), \omega_{\lambda}^{G}(u, T u, T u), \\
\omega_{\lambda}^{G}(u, T u, u), \omega_{\lambda}^{G}(u, u, T u), \\
\omega_{\lambda}^{G}(u, T u, u), \omega_{\lambda}^{G}(u, u, T u), \\
\omega_{\lambda}^{G}(u, T u, T u), \omega_{\lambda}^{G}(u, T u, u), \\
\omega_{\lambda}^{G}(u, u, u), \omega_{\lambda}^{G}(u, T u, T u), \\
\omega_{\lambda}^{G}(u, u, T u), \omega_{\lambda}^{G}(u, u, T u), \\
\omega_{\lambda}^{G}(u, u, T u), \omega_{\lambda}^{G}(u, u, T u), \\
\omega_{\lambda}^{G}(u, u, T u), \omega_{\lambda}^{G}(u, T u, T u)
\end{array}\right\},
$$

for which by condition (4) of Definition 2.1, we have

$$
K_{\lambda}(u, u, u)=\max \left\{\omega_{\lambda}^{G}(u, u, u), \omega_{\lambda}^{G}(u, u, T u), \omega_{\lambda}^{G}(u, T u, T u)\right\}
$$

or

$$
K_{\lambda}(u, u, u)=\max \left\{\omega_{\lambda}^{G}(u, u, T u), \omega_{\lambda}^{G}(u, T u, T u)\right\} .
$$

The modular $G$-metric space is $G$-continuous in each variable for $\lambda>0$ and all the elements of $K_{\lambda}\left(x_{n}, u, u\right)$ converge to $\omega_{\lambda}^{G}(u, u, u), \omega_{\lambda}^{G}(u, u, T u)$ and $\omega_{\lambda}^{G}(u, T u, T u)$ for $\lambda>0$. By condition (4) of Proposition 2.2 and Definition 2.11, we have $\omega_{\lambda}^{G}(u, u, T u) \leq 2 \omega_{\frac{\lambda}{2}}^{G}(u, T u$, $T u) \leq 2 C_{2} \omega_{\lambda}^{G}(u, T u, T u)$ by Definition 2.11, inequality (3.16) gives

$$
\begin{aligned}
\omega_{\lambda}^{G}(u, T u, T u) & \leq \rho \max \left\{\omega_{\lambda}^{G}(u, u, T u), \omega_{\lambda}^{G}(u, T u, T u)\right\} \\
& \leq 2 \rho \omega_{\frac{\lambda}{2}}^{G}(u, T u, T u) \\
& \leq 2 C_{2} \rho \omega_{\lambda}^{G}(u, T u, T u), \quad C_{2} \in\left(0, \frac{1}{2 \rho}\right),
\end{aligned}
$$

which implies that $\omega_{\lambda}^{G}(u, T u, T u) \leq 0$ for all $\lambda>0$, a contradiction. Hence, $T u=u$.

We now show that $T$ has a unique fixed point. Suppose that there exists $v \in X_{\omega^{G}}$ such that $T v=v$ is another fixed point of $T$ in $X_{\omega^{G}}$, so that $u \neq v$; that is $\omega_{\lambda}^{G}(u, v, v)>0$. Indeed suppose, if possible, otherwise, that, for all $\lambda>0$, from inequality (3.1), we have

$$
\omega_{\lambda}^{G}(u, v, v)=\omega_{\lambda}^{G}(T u, T v, T v) \leq \rho K_{\lambda}(u, v, v) \quad \text { for all } u, v \in X_{\omega^{G}}, \lambda>0,
$$


where

$K_{\lambda}(u, v, v)$

$$
\begin{aligned}
& =\max \left\{\begin{array}{l}
\omega_{\lambda}^{G}(u, v, v), \omega_{\lambda}^{G}(u, v, T v), \omega_{\lambda}^{G}(T u, v, v), \omega_{\lambda}^{G}(u, T u, v), \omega_{\lambda}^{G}(v, v, T v), \\
\omega_{\lambda}^{G}(u, T u, T u), \omega_{\lambda}^{G}(u, T v, T v), \frac{\omega_{\lambda}^{G}(v, v, T v)\left[1+\omega_{\lambda}^{G}(u, T u, v)\right]}{1+\omega_{\lambda}^{G}(u, v, v)}, \\
\omega_{\lambda}^{G}(u, T u, v), \omega_{\lambda}^{G}(v, T v, T v), \frac{\omega_{\lambda}^{G}(u, T v, v)\left[1+\omega_{\lambda}^{G}(u, T u, v)\right]}{1+\omega_{\lambda}^{G}(u, v, v)}, \\
\omega_{\lambda}^{G}(v, T u, T v), \frac{\omega_{\lambda}^{G}(u, T v, v)\left[1+\omega_{\lambda}^{G}(u, v, v)\right]}{1+\omega_{\lambda}^{G}(u, v, v)+\omega_{\lambda}^{G}(v, T u, v)}, \omega_{\lambda}^{G}\left(v, T^{2} u, T v\right), \\
\omega_{\lambda}^{G}(v, T v, T v), \frac{\omega_{\lambda}^{G}(u, T v, v)\left[1+\omega_{\lambda}^{G}(u, v, T v)\right]}{1+\omega_{\lambda}^{G}(T u, v, v)+\omega_{\lambda}^{G}(u, v, T v)}, \omega_{\lambda}^{G}(v, T u, T u), \\
\omega_{\lambda}^{G}(v, T v, T v), \omega_{\lambda}^{G}(v, T u, T v), \omega_{\lambda}^{G}\left(v, T^{2} u, T v\right), \omega_{\lambda}^{G}\left(T u, T^{2} u, T v\right), \\
\omega_{\lambda}^{G}\left(T u, T^{2} u, T v\right), \frac{\omega_{\lambda}^{G}(u, v, T v)\left[1+\omega_{\lambda}^{G}(u, v, T v)\right]}{1+\omega_{\lambda}^{G}(T u, v, v)+\omega_{\lambda}^{G}(u, v, T v)}, \\
\frac{\omega_{\lambda}^{G}\left(T^{2} u, T v, T v\right)\left[1+\omega_{\lambda}^{G}(T u, v, v)+\omega_{\lambda}^{G}(T u, v, T v)\right]}{1+\omega_{\lambda}^{G}(T u, v, T v)+\omega_{\lambda}^{G}\left(T^{2} u, T v, T v\right)}
\end{array}\right\} \\
& =\max \left\{\begin{array}{l}
\omega_{\lambda}^{G}(u, v, v), \omega_{\lambda}^{G}(u, v, v), \omega_{\lambda}^{G}(u, v, v), \omega_{\lambda}^{G}(u, u, v), \omega_{\lambda}^{G}(v, v, v), \omega_{\lambda}^{G}(u, u, u), \\
\omega_{\lambda}^{G}(u, v, v), \frac{\omega_{\lambda}^{G}(v, v, v)\left[1+\omega_{\lambda}^{G}(u, u, v)\right]}{1+\omega_{\lambda}^{G}(u, v, v)}, \omega_{\lambda}^{G}(u, u, v), \omega_{\lambda}^{G}(v, v, v), \\
\frac{\omega_{\lambda}^{G}(u, v, v)\left[1+\omega_{\lambda}^{G}(u, u, v)\right]}{1+\omega_{\lambda}^{G}(u, v, v)}, \omega_{\lambda}^{G}(v, u, v), \frac{\omega_{\lambda}^{G}(u, v, v)\left[1+\omega_{\lambda}^{G}(u, v, v)\right]}{1+\omega_{\lambda}^{G}(u, v, v)+\omega_{\lambda}^{G}(v, u, v)}, \\
\omega_{\lambda}^{G}(v, u, v), \omega_{\lambda}^{G}(v, v, v), \frac{\omega_{\lambda}^{G}(u, v, v)\left[1+\omega_{\lambda}^{G}(u, v, v)\right]}{1+\omega_{\lambda}^{G}(u, v, v)+\omega_{\lambda}^{G}(u, v, v)}, \omega_{\lambda}^{G}(v, u, u), \\
\omega_{\lambda}^{G}(v, v, v), \omega_{\lambda}^{G}(v, u, v), \omega_{\lambda}^{G}(v, u, v), \omega_{\lambda}^{G}(u, u, v), \omega_{\lambda}^{G}(u, u, v), \\
\frac{\omega_{\lambda}^{G}(u, v, v)\left[1+\omega_{\lambda}^{G}(u, v, v)\right]}{1+\omega_{\lambda}^{G}(u, v, v)+\omega_{\lambda}^{G}(u, v, v)}, \frac{\omega_{\lambda}^{G}(u, v, v)\left[1+\omega_{\lambda}^{G}(u, v, v)+\omega_{\lambda}^{G}(u, v, v)\right]}{1+\omega_{\lambda}^{G}(u, v, v)+\omega_{\lambda}^{G}(u, v, v)}
\end{array}\right\} .
\end{aligned}
$$

Using condition (4) of Definition 2.1, we clearly see that

$$
\begin{aligned}
K_{\lambda}(u, v, v)=\max \left\{\begin{array}{l}
\omega_{\lambda}^{G}(u, v, v), \omega_{\lambda}^{G}(u, v, v), \omega_{\lambda}^{G}(u, v, v), \omega_{\lambda}^{G}(u, u, v), \omega_{\lambda}^{G}(v, v, v), \\
\omega_{\lambda}^{G}(u, u, u), \omega_{\lambda}^{G}(u, v, v), \omega_{\lambda}^{G}(v, v, v), \omega_{\lambda}^{G}(u, u, v), \omega_{\lambda}^{G}(v, v, v), \\
\omega_{\lambda}^{G}(u, v, v), \omega_{\lambda}^{G}(v, u, v), \omega_{\lambda}^{G}(u, v, v), \omega_{\lambda}^{G}(v, u, v), \omega_{\lambda}^{G}(v, v, v), \\
\omega_{\lambda}^{G}(u, v, v), \omega_{\lambda}^{G}(v, u, u), \omega_{\lambda}^{G}(v, v, v), \omega_{\lambda}^{G}(v, u, v), \omega_{\lambda}^{G}(v, u, v), \\
\omega_{\lambda}^{G}(u, u, v), \omega_{\lambda}^{G}(u, u, v), \omega_{\lambda}^{G}(u, v, v), \omega_{\lambda}^{G}(u, v, v)
\end{array}\right\} \\
=\max \left\{\omega_{\lambda}^{G}(u, v, v), \omega_{\lambda}^{G}(u, u, v)\right\} .
\end{aligned}
$$

Thus from inequality (3.20)

$$
\omega_{\lambda}^{G}(u, v, v) \leq \rho \max \left\{\omega_{\lambda}^{G}(u, v, v), \omega_{\lambda}^{G}(u, u, v)\right\} .
$$


Case 1. If $\max \left\{\omega_{\lambda}^{G}(u, v, v), \omega_{\lambda}^{G}(u, u, v)\right\}$ is $\omega_{\lambda}^{G}(u, v, v)$, then inequality (3.21) becomes

$$
\omega_{\lambda}^{G}(u, v, v) \leq \rho \omega_{\lambda}^{G}(u, v, v)
$$

which implies that $\omega_{\lambda}^{G}(u, v, v) \leq 0, \rho \in(0,1)$.

Case 2. If $\max \left\{\omega_{\lambda}^{G}(u, v, v), \omega_{\lambda}^{G}(u, u, v)\right\}$ is $\omega_{\lambda}^{G}(u, u, v)$, then using condition (3) of Proposition 2.2 and Definition 2.11, inequality (3.21) becomes

$$
\begin{aligned}
\omega_{\lambda}^{G}(u, v, v) & \leq \rho \omega_{\lambda}^{G}(u, u, v) \\
& \leq 2 \rho \omega_{\frac{\lambda}{2}}^{G}(u, v, v) \\
& \leq 2 C_{2} \rho \omega_{\lambda}^{G}(u, v, v),
\end{aligned}
$$

which implies that $\omega_{\lambda}^{G}(u, v, v) \leq 0, C_{2} \in\left(0, \frac{1}{2 \rho}\right)$. Case 1 and Case 2 contradict our initial claim that $\omega_{\lambda}^{G}(u, v, v)>0$ for all $\lambda>0$ hence $T$ has a unique fixed point, i.e. $u=v$.

To see that $T$ is modular $G$-continuous at $u$, let $\left\{x_{n}\right\}_{n \in \mathbb{N}} \subseteq X_{\omega^{G}}$ be a sequence such that $x_{n} \rightarrow u$, then, by taking $x=u, y=x_{n}=z$, inequality (3.1) becomes

$$
\omega_{\lambda}^{G}\left(T u, T x_{n}, T x_{n}\right) \leq \rho K_{\lambda}\left(u, x_{n}, x_{n}\right) \quad \Longrightarrow \quad \omega_{\lambda}^{G}\left(u, T x_{n}, T x_{n}\right) \leq \rho K_{\lambda}\left(u, x_{n}, x_{n}\right),
$$

where

$$
\begin{aligned}
& K_{\lambda}\left(u, x_{n}, x_{n}\right) \\
& =\max \left\{\begin{array}{l}
\omega_{\lambda}^{G}\left(u, x_{n}, x_{n}\right), \omega_{\lambda}^{G}\left(u, x_{n}, T x_{n}\right), \omega_{\lambda}^{G}\left(T u, x_{n}, x_{n}\right), \omega_{\lambda}^{G}\left(u, T u, x_{n}\right), \omega_{\lambda}^{G}\left(x_{n}, x_{n}, T x_{n}\right), \\
\omega_{\lambda}^{G}(u, T u, T u), \omega_{\lambda}^{G}\left(u, T x_{n}, T x_{n}\right), \frac{\omega_{\lambda}^{G}\left(x_{n}, x_{n}, T x_{n}\right)\left[1+\omega_{\lambda}^{G}\left(u, T u, x_{n}\right)\right]}{1+\omega_{\lambda}^{G}\left(u, x_{n}, x_{n}\right)}, \\
\omega_{\lambda}^{G}\left(u, T u, x_{n}\right), \omega_{\lambda}^{G}\left(x_{n}, T x_{n}, T x_{n}\right), \frac{\omega_{\lambda}^{G}\left(u, T x_{n}, x_{n}\right)\left[1+\omega_{\lambda}^{G}\left(u, T u, x_{n}\right)\right]}{1+\omega_{\lambda}^{G}\left(u, x_{n}, x_{n}\right)}, \\
\omega_{\lambda}^{G}\left(x_{n}, T u, T x_{n}\right), \frac{\omega_{\lambda}^{G}\left(u, T x_{n}, x_{n}\right)\left[1+\omega_{\lambda}^{G}\left(u, x_{n}, x_{n}\right)\right]}{1+\omega_{\lambda}^{G}\left(u, x_{n}, x_{n}\right)+\omega_{\lambda}^{G}\left(x_{n}, T u, x_{n}\right)}, \\
\omega_{\lambda}^{G}\left(x_{n}, T^{2} u, T x_{n}\right), \omega_{\lambda}^{G}\left(x_{n}, T x_{n}, T x_{n}\right), \frac{\omega_{\lambda}^{G}\left(u, T x_{n}, x_{n}\right)\left[1+\omega_{\lambda}^{G}\left(u, x_{n}, T x_{n}\right)\right]}{1+\omega_{\lambda}^{G}\left(T u, x_{n}, x_{n}\right)+\omega_{\lambda}^{G}\left(u, x_{n}, T x_{n}\right)}, \\
\omega_{\lambda}^{G}\left(x_{n}, T u, T u\right), \omega_{\lambda}^{G}\left(x_{n}, T x_{n}, T x_{n}\right), \omega_{\lambda}^{G}\left(x_{n}, T u, T x_{n}\right), \omega_{\lambda}^{G}\left(x_{n}, T^{2} u, T x_{n}\right), \\
\omega_{\lambda}^{G}\left(T u, T^{2} u, T x_{n}\right), \omega_{\lambda}^{G}\left(T u, T^{2} u, T x_{n}\right), \frac{\omega_{\lambda}^{G}\left(u, x_{n}, T x_{n}\right)\left[1+\omega_{\lambda}^{G}\left(u, x_{n}, T x_{n}\right)\right]}{1+\omega_{\lambda}^{G}\left(T u, x_{n}, x_{n}\right)+\omega_{\lambda}^{G}\left(u, x_{n}, T x_{n}\right)} \\
\frac{\omega_{\lambda}^{G}\left(T^{2} u, T x_{n}, T x_{n}\right)\left[1+\omega_{\lambda}^{G}\left(T u, x_{n}, x_{n}\right)+\omega_{\lambda}^{G}\left(T u, x_{n}, T x_{n}\right)\right]}{1+\omega_{\lambda}^{G}\left(T u, x_{n}, T x_{n}\right)+\omega_{\lambda}^{G}\left(T^{2} u, T x_{n}, T x_{n}\right)}
\end{array}\right\}
\end{aligned}
$$




$$
=\max \left\{\begin{array}{l}
\omega_{\lambda}^{G}\left(u, x_{n}, x_{n}\right), \omega_{\lambda}^{G}\left(u, x_{n}, T x_{n}\right), \omega_{\lambda}^{G}\left(u, x_{n}, x_{n}\right), \omega_{\lambda}^{G}\left(u, u, x_{n}\right), \omega_{\lambda}^{G}\left(x_{n}, x_{n}, T x_{n}\right), \\
\omega_{\lambda}^{G}(u, u, u), \omega_{\lambda}^{G}\left(u, T x_{n}, T x_{n}\right), \frac{\omega_{\lambda}^{G}\left(x_{n}, x_{n}, T x_{n}\right)\left[1+\omega_{\lambda}^{G}\left(u, u, x_{n}\right)\right]}{1+\omega_{\lambda}^{G}\left(u, x_{n}, x_{n}\right)}, \\
\omega_{\lambda}^{G}\left(u, u, x_{n}\right), \omega_{\lambda}^{G}\left(x_{n}, T x_{n}, T x_{n}\right), \frac{\omega_{\lambda}^{G}\left(u, T x_{n}, x_{n}\right)\left[1+\omega_{\lambda}^{G}\left(u, u, x_{n}\right)\right]}{1+\omega_{\lambda}^{G}\left(u, x_{n}, x_{n}\right)}, \\
\omega_{\lambda}^{G}\left(x_{n}, u, T x_{n}\right), \frac{\omega_{\lambda}^{G}\left(u, T x_{n}, x_{n}\right)\left[1+\omega_{\lambda}^{G}\left(u, x_{n}, x_{n}\right)\right]}{1+\omega_{\lambda}^{G}\left(u, x_{n}, x_{n}\right)+\omega_{\lambda}^{G}\left(x_{n}, u, x_{n}\right)} \\
\omega_{\lambda}^{G}\left(x_{n}, u, u\right), \omega_{\lambda}^{G}\left(x_{n}, T x_{n}, T x_{n}\right), \omega_{\lambda}^{G}\left(x_{n}, T x_{n}, T x_{n}\right), \frac{\omega_{\lambda}^{G}\left(u, T x_{n}, x_{n}\right)\left[1+\omega_{\lambda}^{G}\left(u, x_{n}, T x_{n}\right)\right]}{1+\omega_{\lambda}^{G}\left(u, x_{n}, x_{n}\right)+\omega_{\lambda}^{G}\left(u, x_{n}, T x_{n}\right)}, \\
\omega_{\lambda}^{G}\left(u, u, T x_{n}\right), \omega_{\lambda}^{G}\left(u, u, T x_{n}\right), \frac{\omega_{\lambda}^{G}\left(u, x_{n}, T x_{n}\right)\left[1+\omega_{\lambda}^{G}\left(u, x_{n}, T x_{n}\right)\right]}{1+\omega_{\lambda}^{G}\left(u, x_{n}, x_{n}\right)+\omega_{\lambda}^{G}\left(u, x_{n}, T x_{n}\right)}, \\
\frac{\omega_{\lambda}^{G}\left(u, T x_{n}, T x_{n}\right)\left[1+\omega_{\lambda}^{G}\left(u, x_{n}, x_{n}\right)+\omega_{\lambda}^{G}\left(u, x_{n}, T x_{n}\right)\right]}{1+\omega_{\lambda}^{G}\left(u, x_{n}, T x_{n}\right)+\omega_{\lambda}^{G}\left(u, T x_{n}, T x_{n}\right)}
\end{array}\right\} .
$$

Since $T u=u \Longrightarrow T^{2} u=T(T u)=T u=u$ and $\frac{\omega_{\lambda}^{G}\left(u, u, x_{n}\right)}{\omega_{\lambda}^{G}\left(u, x_{n}, x_{n}\right)} \leq 1$, we have

$$
K_{\lambda}\left(u, x_{n}, x_{n}\right)=\max \left\{\begin{array}{l}
\omega_{\lambda}^{G}\left(u, x_{n}, x_{n}\right), \omega_{\lambda}^{G}\left(u, x_{n}, T x_{n}\right), \omega_{\lambda}^{G}\left(u, x_{n}, x_{n}\right), \\
\omega_{\lambda}^{G}\left(u, u, x_{n}\right), \omega_{\lambda}^{G}\left(x_{n}, x_{n}, T x_{n}\right), \omega_{\lambda}^{G}(u, u, u), \\
\omega_{\lambda}^{G}\left(u, T x_{n}, T x_{n}\right), \omega_{\lambda}^{G}\left(x_{n}, x_{n}, T x_{n}\right), \omega_{\lambda}^{G}\left(u, u, x_{n}\right), \\
\omega_{\lambda}^{G}\left(x_{n}, T x_{n}, T x_{n}\right), \omega_{\lambda}^{G}\left(u, T x_{n}, x_{n}\right), \omega_{\lambda}^{G}\left(x_{n}, u, T x_{n}\right), \\
\omega_{\lambda}^{G}\left(u, T x_{n}, x_{n}\right), \omega_{\lambda}^{G}\left(x_{n}, u, T x_{n}\right), \omega_{\lambda}^{G}\left(x_{n}, T x_{n}, T x_{n}\right), \\
\omega_{\lambda}^{G}\left(u, T x_{n}, x_{n}\right), \omega_{\lambda}^{G}\left(x_{n}, u, u\right), \omega_{\lambda}^{G}\left(x_{n}, T x_{n}, T x_{n}\right), \\
\omega_{\lambda}^{G}\left(x_{n}, u, T x_{n}\right), \omega_{\lambda}^{G}\left(x_{n}, u, T x_{n}\right), \omega_{\lambda}^{G}\left(u, u, T x_{n}\right), \\
\omega_{\lambda}^{G}\left(u, u, T x_{n}\right), \omega_{\lambda}^{G}\left(u, x_{n}, T x_{n}\right), \omega_{\lambda}^{G}\left(u, T x_{n}, T x_{n}\right)
\end{array}\right\} .
$$

So,

$$
\begin{gathered}
K_{\lambda}\left(u, x_{n}, x_{n}\right)=\max \left\{\begin{array}{c}
\omega_{\lambda}^{G}\left(u, x_{n}, x_{n}\right), \omega_{\lambda}^{G}\left(u, x_{n}, T x_{n}\right), \\
\omega_{\lambda}^{G}\left(u, u, x_{n}\right), \omega_{\lambda}^{G}\left(x_{n}, x_{n}, T x_{n}\right), \\
\omega_{\lambda}^{G}(u, u, u), \omega_{\lambda}^{G}\left(u, T x_{n}, T x_{n}\right), \\
\omega_{\lambda}^{G}\left(x_{n}, T x_{n}, T x_{n}\right), \omega_{\lambda}^{G}\left(u, u, T x_{n}\right)
\end{array}\right\}, \\
K_{\lambda}\left(u, x_{n}, x_{n}\right)=\max \left\{\begin{array}{l}
\omega_{\lambda}^{G}\left(u, x_{n}, x_{n}\right), \omega_{\lambda}^{G}\left(u, x_{n}, T x_{n}\right), \omega_{\lambda}^{G}\left(u, u, x_{n}\right), \omega_{\lambda}^{G}\left(x_{n}, x_{n}, T x_{n}\right), \\
\omega_{\lambda}^{G}\left(u, T x_{n}, T x_{n}\right), \omega_{\lambda}^{G}\left(x_{n}, T x_{n}, T x_{n}\right), \omega_{\lambda}^{G}\left(u, u, T x_{n}\right)
\end{array}\right\} .
\end{gathered}
$$

Now we consider the following cases. 
Case 1 . If $K_{\lambda}\left(u, x_{n}, x_{n}\right)=\omega_{\lambda}^{G}\left(u, x_{n}, x_{n}\right)$, then inequality (3.24) becomes

$$
\omega_{\lambda}^{G}\left(u, T x_{n}, T x_{n}\right) \leq \rho \omega_{\lambda}^{G}\left(u, x_{n}, x_{n}\right),
$$

for $\rho \in(0,1)$.

Case 2. If $K_{\lambda}\left(u, x_{n}, x_{n}\right)=\omega_{\lambda}^{G}\left(u, x_{n}, T x_{n}\right)$, using conditions (1), (5) of Definition 2.1, conditions (6), (3) of Proposition 2.2 and Definition 2.11, then inequality (3.24) becomes

$$
\begin{aligned}
\omega_{\lambda}^{G}\left(u, T x_{n}, T x_{n}\right) \leq & \rho \omega_{\lambda}^{G}\left(u, x_{n}, T x_{n}\right) \\
\leq & \rho\left\{\omega_{\frac{\lambda}{2}}^{G}\left(u, x_{n}, x_{n}\right)+\omega_{\frac{\lambda}{4}}^{G}\left(x_{n}, x_{n}, x_{n}\right)+\omega_{\frac{\lambda}{4}}^{G}\left(x_{n}, x_{n}, T x_{n}\right)\right\} \\
& =\rho\left\{\omega_{\frac{\lambda}{2}}^{G}\left(u, x_{n}, x_{n}\right)+\omega_{\frac{\lambda}{4}}^{G}\left(x_{n}, x_{n}, T x_{n}\right)\right\} \\
& \leq \rho\left\{C_{2} \omega_{\lambda}^{G}\left(u, x_{n}, x_{n}\right)+C_{4} \omega_{\lambda}^{G}\left(x_{n}, x_{n}, T x_{n}\right)\right\} \\
\leq & \rho\left\{C_{2} \omega_{\lambda}^{G}\left(u, x_{n}, x_{n}\right)+2 C_{4} \omega_{\frac{\lambda}{2}}^{G}\left(x_{n}, T x_{n}, T x_{n}\right)\right\} \\
& \leq \rho\left\{C_{2} \omega_{\lambda}^{G}\left(u, x_{n}, x_{n}\right)+2 C_{2} C_{4} \omega_{\lambda}^{G}\left(x_{n}, T x_{n}, T x_{n}\right)\right\} \\
& b u t \omega_{\lambda}^{G}\left(x_{n}, T x_{n}, T x_{n}\right) \leq \omega_{\lambda}^{G}\left(x_{n}, u, u\right)+\omega_{\lambda}^{G}\left(u, T x_{n}, T x_{n}\right) \\
\leq & \rho\left\{C_{2} \omega_{\lambda}^{G}\left(u, x_{n}, x_{n}\right)+2 C_{2} C_{4}\left(\omega_{\lambda}^{G}\left(x_{n}, u, u\right)+\omega_{\lambda}^{G}\left(u, T x_{n}, T x_{n}\right)\right)\right\} \\
\leq & \rho\left\{C_{2} \omega_{\lambda}^{G}\left(u, x_{n}, x_{n}\right)+2 C_{2} C_{4}\left(C_{2} \omega_{\lambda}^{G}\left(x_{n}, u, u\right)+C_{2} \omega_{\lambda}^{G}\left(u, T x_{n}, T x_{n}\right)\right)\right\} \\
\leq & \rho\left\{C_{2} \omega_{\lambda}^{G}\left(u, x_{n}, x_{n}\right)+2 C_{2}^{2} C_{4}\left(2 \omega_{\frac{\lambda}{2}}^{G}\left(u, x_{n}, x_{n}\right)+\omega_{\lambda}^{G}\left(u, T x_{n}, T x_{n}\right)\right)\right\} \\
\leq & \rho\left\{C_{2} \omega_{\lambda}^{G}\left(u, x_{n}, x_{n}\right)+2 C_{2}^{2} C_{4}\left(2 C_{2} \omega_{\lambda}^{G}\left(u, x_{n}, x_{n}\right)+\omega_{\lambda}^{G}\left(u, T x_{n}, T x_{n}\right)\right)\right\} \\
= & \rho\left(C_{2} \omega_{\lambda}^{G}\left(u, x_{n}, x_{n}\right)+4 C_{2}^{3} C_{4} \omega_{\lambda}^{G}\left(u, x_{n}, x_{n}\right)+2 C_{2}^{2} C_{4} \omega_{\lambda}^{G}\left(u, T x_{n}, T x_{n}\right)\right) \\
= & \rho\left(C_{2}\left(1+4 C_{2}^{2} C_{4}\right) \omega_{\lambda}^{G}\left(u, x_{n}, x_{n}\right)+2 C_{2}^{2} C_{4} \omega_{\lambda}^{G}\left(u, T x_{n}, T x_{n}\right)\right),
\end{aligned}
$$

so that

$$
\omega_{\lambda}^{G}\left(u, T x_{n}, T x_{n}\right) \leq \frac{\rho C_{2}\left(1+4 C_{2}^{2} C_{4}\right)}{1-2 \rho C_{2}^{2} C_{4}} \omega_{\lambda}^{G}\left(u, x_{n}, x_{n}\right)
$$

therefore, we have

$$
\omega_{\lambda}^{G}\left(u, T x_{n}, T x_{n}\right) \leq \theta_{j} \omega_{\lambda}^{G}\left(u, x_{n}, x_{n}\right)
$$

where $\theta_{j}=\frac{\rho C_{2}\left(1+4 C_{2}^{2} C_{4}\right)}{1-j \rho C_{2}^{2} C_{4}}<1$ and $C_{2}^{2} C_{4} \in\left(0, \frac{1}{j \rho}\right)$, where $j=2$.

Case 3. If $K_{\lambda}\left(u, x_{n}, x_{n}\right)=\omega_{\lambda}^{G}\left(u, u, x_{n}\right)$, by condition (3) of Proposition 2.2 and Definition 2.11, then inequality (3.24) becomes

$$
\begin{aligned}
\omega_{\lambda}^{G}\left(u, T x_{n}, T x_{n}\right) & \leq \rho \omega_{\lambda}^{G}\left(u, u, x_{n}\right) \\
& \leq 2 \rho \omega_{\frac{\lambda}{2}}^{G}\left(u, x_{n}, x_{n}\right)
\end{aligned}
$$




$$
\begin{aligned}
& \leq 2 \rho C_{2} \omega_{\lambda}^{G}\left(u, x_{n}, x_{n}\right) \\
& \leq \delta_{j} \omega_{\lambda}^{G}\left(u, x_{n}, x_{n}\right),
\end{aligned}
$$

where $\delta_{j}=j \rho C_{2}<1$ and $C_{2} \in\left(0, \frac{1}{j \rho}\right)$ for $j=2$.

Case 4. If $K_{\lambda}\left(u, x_{n}, x_{n}\right)=\omega_{\lambda}^{G}\left(x_{n}, x_{n}, T x_{n}\right)$, using condition (3) of Proposition 2.2, condition (5) of Definition 2.1 and Definition 2.11, then inequality (3.24) becomes

$$
\begin{aligned}
\omega_{\lambda}^{G}\left(u, T x_{n}, T x_{n}\right) & \leq \rho \omega_{\lambda}^{G}\left(x_{n}, x_{n}, T x_{n}\right) \\
& \leq 2 \rho \omega_{\frac{\lambda}{2}}^{G}\left(x_{n}, T x_{n}, T x_{n}\right) \\
& \leq 2 C_{2} \rho \omega_{\lambda}^{G}\left(x_{n}, T x_{n}, T x_{n}\right) \\
& \leq 2 C_{2} \rho\left(\omega_{\frac{\lambda}{2}}^{G}\left(x_{n}, u, u\right)+\omega_{\frac{\lambda}{2}}^{G}\left(u, T x_{n}, T x_{n}\right)\right) \\
& \leq 2 C_{2}^{2} \rho\left(\omega_{\lambda}^{G}\left(x_{n}, u, u\right)+\omega_{\lambda}^{G}\left(u, T x_{n}, T x_{n}\right)\right) \\
& \leq 2 C_{2}^{2} \rho\left(2 \omega_{\frac{\lambda}{2}}^{G}\left(u, x_{n}, x_{n}\right)+\omega_{\lambda}^{G}\left(u, T x_{n}, T x_{n}\right)\right) \\
& \leq 2 C_{2}^{2} \rho\left(2 C_{2} \omega_{\lambda}^{G}\left(u, x_{n}, x_{n}\right)+\omega_{\lambda}^{G}\left(u, T x_{n}, T x_{n}\right)\right),
\end{aligned}
$$

so that

$$
\omega_{\lambda}^{G}\left(u, T x_{n}, T x_{n}\right) \leq \beta_{j} \omega_{\lambda}^{G}\left(u, x_{n}, x_{n}\right)
$$

where $\beta_{j}=\frac{4 \rho C_{2}^{3}}{1-j \rho C_{2}^{2}}<1$ and $C_{2}^{2} \in\left(0, \frac{1}{j \rho}\right)$ for $j=2$.

Case 5. If $K_{\lambda}\left(u, x_{n}, x_{n}\right)=\omega_{\lambda}^{G}\left(u, T x_{n}, T x_{n}\right)$, using condition (6) of Proposition 2.2 and Case 4 , then inequality (3.24) becomes

$$
\begin{aligned}
\omega_{\lambda}^{G}\left(u, T x_{n}, T x_{n}\right) \leq & \rho \omega_{\lambda}^{G}\left(u, T x_{n}, T x_{n}\right) \\
\leq & \rho\left(\omega_{\frac{\lambda}{2}}^{G}\left(u, x_{n}, x_{n}\right)+\omega_{\frac{\lambda}{4}}^{G}\left(x_{n}, x_{n}, T x_{n}\right)+\omega_{\frac{\lambda}{4}}^{G}\left(x_{n}, x_{n}, T x_{n}\right)\right) \\
& =\rho\left(\omega_{\frac{\lambda}{2}}^{G}\left(u, x_{n}, x_{n}\right)+2 \omega_{\frac{\lambda}{4}}^{G}\left(x_{n}, x_{n}, T x_{n}\right)\right) \\
\leq & \rho\left(C_{2} \omega_{\lambda}^{G}\left(u, x_{n}, x_{n}\right)+2 C_{4} \omega_{\lambda}^{G}\left(x_{n}, x_{n}, T x_{n}\right)\right) \\
\leq & \rho\left(C_{2} \omega_{\lambda}^{G}\left(u, x_{n}, x_{n}\right)+6 C_{4} C_{2}^{3} \omega_{\lambda}^{G}\left(u, x_{n}, x_{n}\right)\right. \\
& \left.+4 C_{4} C_{2}^{2} \omega_{\lambda}^{G}\left(x_{n}, T x_{n}, T x_{n}\right)\right) .
\end{aligned}
$$

Therefore, from inequality (3.30), we get

$$
\omega_{\lambda}^{G}\left(x_{n}, T x_{n}, T x_{n}\right) \leq \frac{\rho C_{2}\left(1+6 C_{4} C_{2}^{2}\right)}{1-4 \rho C_{4} C_{2}^{2}} \omega_{\lambda}^{G}\left(u, x_{n}, x_{n}\right),
$$

so that $C_{2}^{2} C_{4} \in\left(0, \frac{1}{j \rho}\right)$, where $j=4$.

Case 6. If $K_{\lambda}\left(u, x_{n}, x_{n}\right)=\omega_{\lambda}^{G}\left(x_{n}, T x_{n}, T x_{n}\right)$, using property (5) of Definition 2.1, condition (3) of Proposition 2.2 and Definition 2.11, then inequality (3.24) becomes

$$
\begin{aligned}
\omega_{\lambda}^{G}\left(u, T x_{n}, T x_{n}\right) & \leq \rho \omega_{\lambda}^{G}\left(x_{n}, T x_{n}, T x_{n}\right) \\
& \leq \rho\left(\omega_{\frac{\lambda}{2}}^{G}\left(x_{n}, u, u\right)+\omega_{\frac{\lambda}{2}}^{G}\left(u, T x_{n}, T x_{n}\right)\right)
\end{aligned}
$$




$$
\begin{aligned}
& \leq \rho C_{2}\left(\omega_{\lambda}^{G}\left(x_{n}, u, u\right)+\omega_{\lambda}^{G}\left(u, T x_{n}, T x_{n}\right)\right) \\
& \leq \rho C_{2}\left(2 \omega_{\frac{\lambda}{2}}^{G}\left(u, x_{n}, x_{n}\right)+\omega_{\lambda}^{G}\left(u, T x_{n}, T x_{n}\right)\right) \\
& \leq \rho C_{2}\left(2 C_{2} \omega_{\lambda}^{G}\left(u, x_{n}, x_{n}\right)+\omega_{\lambda}^{G}\left(u, T x_{n}, T x_{n}\right)\right),
\end{aligned}
$$

so that

$$
\omega_{\lambda}^{G}\left(u, T x_{n}, T x_{n}\right) \leq \frac{2 \rho C_{2}^{2}}{1-\rho C_{2}} \omega_{\lambda}^{G}\left(u, x_{n}, x_{n}\right), \quad \text { for some } C_{2}>0,
$$

so that $C_{2} \in\left(0, \frac{1}{j \rho}\right)$, where $j=1$.

Case 7. If $K_{\lambda}\left(u, x_{n}, x_{n}\right)=\omega_{\lambda}^{G}\left(u, u, T x_{n}\right)$, using conditions (6), (3) of Proposition 2.2 and Definition 2.11, condition (5) of Definition 2.1, then inequality (3.24) becomes

$$
\begin{aligned}
\omega_{\lambda}^{G} & \left(u, T x_{n}, T x_{n}\right) \\
& \leq \rho \omega_{\lambda}^{G}\left(u, u, T x_{n}\right) \\
& \leq \rho\left\{\omega_{\lambda}^{G}\left(u, x_{n}, x_{n}\right)+\omega_{\frac{\lambda}{4}}^{G}\left(u, x_{n}, x_{n}\right)+\omega_{\lambda}^{G}\left(x_{n}, x_{n}, T x_{n}\right)\right\} \\
& \leq \rho\left\{C_{2} \omega_{\lambda}^{G}\left(u, x_{n}, x_{n}\right)+C_{4} \omega_{\lambda}^{G}\left(u, x_{n}, x_{n}\right)+C_{4} \omega_{\lambda}^{G}\left(x_{n}, x_{n}, T x_{n}\right)\right\} \\
& =\rho\left\{\left(C_{2}+C_{4}\right) \omega_{\lambda}^{G}\left(u, x_{n}, x_{n}\right)+C_{4} \omega_{\lambda}^{G}\left(x_{n}, x_{n}, T x_{n}\right)\right\} \\
& \leq \rho\left\{\left(C_{2}+C_{4}\right) \omega_{\lambda}^{G}\left(u, x_{n}, x_{n}\right)+2 C_{4} \omega_{\lambda}^{G}\left(x_{n}, T x_{n}, T x_{n}\right)\right\} \\
& \leq \rho\left\{\left(C_{2}+C_{4}\right) \omega_{\lambda}^{G}\left(u, x_{n}, x_{n}\right)+2 C_{2} C_{4} \omega_{\lambda}^{G}\left(x_{n}, T x_{n}, T x_{n}\right)\right\} \\
& \leq \rho\left\{\left(C_{2}+C_{4}\right) \omega_{\lambda}^{G}\left(u, x_{n}, x_{n}\right)+2 C_{2} C_{4}\left(\omega_{\lambda}^{G}\left(x_{n}, u, u\right)+\omega_{\lambda}^{G}\left(u, T x_{n}, T x_{n}\right)\right)\right\} \\
& \leq \rho\left\{\left(C_{2}+C_{4}\right) \omega_{\lambda}^{G}\left(u, x_{n}, x_{n}\right)+2 C_{2}^{2} C_{4}\left(\omega_{\lambda}^{G}\left(x_{n}, u, u\right)+\omega_{\lambda}^{G}\left(u, T x_{n}, T x_{n}\right)\right)\right\} \\
& \leq \rho\left\{\left(C_{2}+C_{4}\right) \omega_{\lambda}^{G}\left(u, x_{n}, x_{n}\right)+2 C_{2}^{2} C_{4}\left(2 \omega_{\frac{\lambda}{2}}^{G}\left(u, x_{n}, x_{n}\right)+\omega_{\lambda}^{G}\left(u, T x_{n}, T x_{n}\right)\right)\right\} \\
& \leq \rho\left\{\left(C_{2}+C_{4}\right) \omega_{\lambda}^{G}\left(u, x_{n}, x_{n}\right)+2 C_{2}^{2} C_{4}\left(2 C_{2} \omega_{\lambda}^{G}\left(u, x_{n}, x_{n}\right)+\omega_{\lambda}^{G}\left(u, T x_{n}, T x_{n}\right)\right)\right\} \\
& =\rho\left(\left(C_{2}+C_{4}\right) \omega_{\lambda}^{G}\left(u, x_{n}, x_{n}\right)+4 C_{2}^{3} C_{4} \omega_{\lambda}^{G}\left(u, x_{n}, x_{n}\right)+2 C_{2}^{2} C_{4} \omega_{\lambda}^{G}\left(u, T x_{n}, T x_{n}\right)\right) \\
& =\rho\left(\left(C_{2}+C_{4}+4 C_{2}^{3} C_{4}\right) \omega_{\lambda}^{G}\left(u, x_{n}, x_{n}\right)+2 C_{2}^{2} C_{4} \omega_{\lambda}^{G}\left(u, T x_{n}, T x_{n}\right)\right),
\end{aligned}
$$

so that inequality (3.33) becomes

$$
\omega_{\lambda}^{G}\left(u, T x_{n}, T x_{n}\right) \leq \eta_{j} \omega_{\lambda}^{G}\left(u, x_{n}, x_{n}\right)
$$

where $\eta_{j}=\frac{\rho\left(C_{2}+C_{4}\left(1+4 C_{2}^{3}\right)\right)}{1-2 \rho C_{2}^{2} C_{4}}<1$, and $C_{2}^{2} C_{4} \in\left(0, \frac{1}{j \rho}\right)$, where $j=2$. Since $x_{n} \rightarrow u, n \rightarrow \infty$, in all the cases, we have $T x_{n}=u=T u$ as $n \rightarrow \infty$, showing that $T$ is modular G-continuous at the fixed point $u$.

Remark 3.1 If the statement of Theorem 3.1 holds without non-symmetric condition and the maximum in inequality $(3.1)$ is $\omega_{\lambda}^{G}(x, y, z)$, then inequality (3.1) becomes

$$
\omega_{\lambda}^{G}(T x, T y, T z) \leq \rho \omega_{\lambda}^{G}(x, y, z), \quad \forall x, y, z \in X_{\omega^{G}}, \rho \in(0,1), \lambda>0 .
$$

This generalized Theorem 3.2 in [35]. 
Remark 3.2 If the statement of Theorem 3.1 hold without $\Delta_{3}$-type conditions and the maximum in inequality (3.1) becomes

$$
\omega_{\lambda}^{G}(T x, T y, T z) \leq \rho K_{\lambda}(x, y, z) \quad \text { for all } x, y, z \in X_{\omega^{G}}, \lambda>0,
$$

where

$$
\begin{aligned}
& K_{\lambda}(x, y, z) \\
& =\max \left\{\begin{array}{l}
\omega_{\lambda}^{G}(x, y, z), \omega_{\lambda}^{G}(x, T x, y), \omega_{\lambda}^{G}(x, T x, T x), \omega_{\lambda}^{G}(x, T y, T y), \omega_{\lambda}^{G}(x, T x, z), \\
\omega_{\lambda}^{G}(y, T y, T y), \omega_{\lambda}^{G}(y, T x, T y), \omega_{\lambda}^{G}\left(y, T^{2} x, T y\right), \omega_{\lambda}^{G}(y, T z, T z), \\
\omega_{\lambda}^{G}(z, T x, T x), \omega_{\lambda}^{G}(z, T z, T z), \omega_{\lambda}^{G}(z, T x, T y), \omega_{\lambda}^{G}\left(z, T^{2} x, T z\right), \\
\omega_{\lambda}^{G}\left(T x, T^{2} x, T y\right), \omega_{\lambda}^{G}\left(T x, T^{2} x, T z\right)
\end{array}\right\} .
\end{aligned}
$$

Then $T$ has a unique fixed point in $X_{\omega^{G}}$ and is modular $G$-continuous at the fixed point (say $u$ ).This is Theorem 3.1 in [30], Theorem 2.1 in [12] in the setting of modular G-metric space.

Remark 3.3 Remark 3.2 can be extended a little as follows: If the statement of Theorem 3.1 holds without $\Delta_{3}$-type conditions and the maximum in inequality (3.1) becomes

$$
\omega_{\lambda}^{G}(T x, T y, T z) \leq \rho K_{\lambda}(x, y, z) \quad \text { for all } x, y, z \in X_{\omega^{G}}, \lambda>0
$$

where

$$
\begin{aligned}
& K_{\lambda}(x, y, z) \\
& =\max \left\{\begin{array}{l}
\omega_{\lambda}^{G}(x, y, z), \omega_{\lambda}^{G}(x, z, T y), \omega_{\lambda}^{G}(T x, y, z), \\
\omega_{\lambda}^{G}(x, T x, y), \omega_{\lambda}^{G}(y, z, T y), \omega_{\lambda}^{G}(x, T x, T x), \\
\omega_{\lambda}^{G}(x, T y, T y), \omega_{\lambda}^{G}(x, T x, z), \omega_{\lambda}^{G}(y, T y, T y), \\
\omega_{\lambda}^{G}(y, T x, T y), \omega_{\lambda}^{G}\left(y, T^{2} x, T y\right), \omega_{\lambda}^{G}(y, T z, T z), \\
\omega_{\lambda}^{G}(z, T x, T x), \omega_{\lambda}^{G}(z, T z, T z), \omega_{\lambda}^{G}(z, T x, T y), \\
\omega_{\lambda}^{G}\left(z, T^{2} x, T z\right), \omega_{\lambda}^{G}\left(T x, T^{2} x, T y\right), \omega_{\lambda}^{G}\left(T x, T^{2} x, T z\right)
\end{array}\right\},
\end{aligned}
$$

then $T$ has a unique fixed point in $X_{\omega^{G}}$ and is modular G-continuous at the fixed point (say $u$ ). This is an extension of Theorem 3.1 in [30] in the setting of modular G-metric space.

We give the following examples to support Theorem 3.1.

Example 3.1 Suppose that $X=[0,1] \cup\{\infty\} \subseteq \overline{\mathbb{R}}$ with the modular $G$-metric for $\lambda>0$ defined by $\omega_{\lambda}^{G}(x, y, z)=\frac{G(x, y, z)}{\lambda}$ so that $G(x, y, z)=\max \{|x-y|,|y-z|,|z-x|\}$ for all $x, y, z \in X_{\omega^{G}}$. Define a self map $T: X_{\omega^{G}} \rightarrow X_{\omega^{G}}$ by $T x=\frac{x^{2}}{2}, \forall x \in X_{\omega^{G}}$, then: 
(i) $\left(X, \omega^{G}\right)$ is a $G$-complete modular $G$-metric space. It suffices to show that any arbitrary sequence we pick in modular G-metric space, i.e. $\left\{x_{n}\right\}_{n \in \mathbb{N}} \subseteq X_{\omega^{G}}$ will be a modular $G$ Cauchy sequence; that is if for every $\epsilon>0$, there exists $n_{\epsilon} \in \mathbb{N}$ such that $\omega_{\lambda}^{G}\left(x_{n}, x_{m}, x_{l}\right)<\epsilon$ for all $n, m, l \geq n_{\epsilon}$ and $\lambda>0$. Recall that a modular $G$-metric space $X_{\omega^{G}}$ is said to be $G$ complete if every G-Cauchy sequence in $X_{\omega^{G}}$ is $G$-convergent in $X_{\omega^{G}}$. Indeed, let $\left(X, \omega^{G}\right)$ be a modular $G$-metric space and let $\left\{x_{n}\right\}_{n \in \mathbb{N}} \subseteq X_{\omega^{G}}$ be a sequence that converges to $u \in X_{\omega^{G}}$. Let $\epsilon>0$ be arbitrary. By definition of the modular $G$-metric and for all $\lambda>0$, there exists $n_{\epsilon} \in \mathbb{N}, C_{2}>0$ such that $\omega_{\lambda}^{G}\left(x_{n}, x_{m}, u\right)<\frac{\epsilon}{3 C_{2}}$ for all $n, m \geq n_{\epsilon}$ and $\omega_{\lambda}^{G}\left(x_{n}, x_{n}, u\right)<\frac{\epsilon}{3 C_{2}^{2}}$ for all $n, m \geq n_{\epsilon}$ for all $\lambda>0$. Using conditions (4), (5) of Definition 2.1 and condition (3) of Proposition 2.2 and Definition 2.11 or in particular the Remark 2.4, we have, for all $n, m, l>n_{\epsilon}$,

$$
\begin{aligned}
\omega_{\lambda}^{G}\left(x_{n}, x_{m}, x_{l}\right) & \leq \omega_{\frac{\lambda}{2}}^{G}\left(x_{n}, u, u\right)+\omega_{\frac{\lambda}{2}}^{G}\left(u, x_{m}, x_{l}\right) \\
& \leq C_{2} \omega_{\lambda}^{G}\left(x_{n}, u, u\right)+C_{2} \omega_{\lambda}^{G}\left(u, x_{m}, x_{l}\right) \\
& =C_{2}\left[\omega_{\lambda}^{G}\left(x_{n}, u, u\right)+\omega_{\lambda}^{G}\left(u, x_{m}, x_{l}\right)\right] \\
& \leq C_{2}\left[2 \omega_{\frac{\lambda}{2}}^{G}\left(x_{n}, x_{n}, u\right)+\omega_{\lambda}^{G}\left(u, x_{m}, x_{l}\right)\right] \\
& \leq C_{2}\left[2 C_{2} \omega_{\lambda}^{G}\left(x_{n}, x_{n}, u\right)+\omega_{\lambda}^{G}\left(u, x_{m}, x_{l}\right)\right] \\
& =2 C_{2}^{2} \omega_{\lambda}^{G}\left(x_{n}, x_{n}, u\right)+C_{2} \omega_{\lambda}^{G}\left(u, x_{m}, x_{l}\right) \\
& <2 C_{2}^{2} \frac{\epsilon}{3 C_{2}^{2}}+C_{2} \frac{\epsilon}{3 C_{2}} \\
& =\frac{2 \epsilon}{3}+\frac{\epsilon}{3}=\epsilon .
\end{aligned}
$$

Thus $\omega_{\lambda}^{G}\left(x_{n}, x_{m}, x_{l}\right)<\epsilon$ for all $n, m, l>n_{\epsilon}$ and $\lambda>0$. Therefore, $\left\{x_{n}\right\}_{n \geq 1}$ is a modular GCauchy sequence. Let $\left\{x_{n}\right\}_{n \geq 1}$ be a modular $G$-Cauchy sequence. Since every modular G-Cauchy sequence is modular G-bounded, $\left\{x_{n}\right\}_{n \geq 1}$ is modular G-bounded. Since every modular $G$-bounded sequence has a limit point, $\left\{x_{n}\right\}_{n \geq 1}$ has a limit point $u$. Furthermore, we show that $\left\{x_{n}\right\}_{n \geq 1}$ converges to $u$. Let $\epsilon>0$ be given. Since $\left\{x_{n}\right\}_{n \geq 1}$ is a modular $G$ Cauchy sequence, there exists a positive integer, $m$, and $C_{2}>0$ such that $\omega_{\lambda}^{G}\left(x_{n}, x_{m}, x_{m}\right)<$ $\frac{\epsilon}{3 C_{2}} \forall n \geq m$ and for all $\lambda>0$. Since $u$ is a limit point of $\left\{x_{n}\right\}_{n \geq 1}$, every neighborhood of $u$ contains infinitely many times of $\left\{x_{n}\right\}_{n \geq 1}$ which implies that $x_{n} \in\left(u-\frac{\epsilon}{3 C_{2}}, u+\frac{\epsilon}{3 C_{2}}\right)$ for infinitely many values of $n$. In particular, we can find a positive integer $k>m, \lambda>0$ and $C_{4}>0$ such that $\omega_{\lambda}^{G}\left(x_{k}, x_{k}, u\right)<\frac{\epsilon}{3 C_{4}} \forall k \geq m$ i.e. $x_{k} \in\left(u-\frac{\epsilon}{3 C_{4}}, u+\frac{\epsilon}{3 C_{4}}\right)$. Also, since $k>m$, we have $\omega_{\lambda}^{G}\left(x_{k}, x_{m}, x_{m}\right)<\frac{\epsilon}{3 C_{4}}$. Now by condition (6) of Proposition 2.2 and Definition 2.11 we have

$$
\begin{aligned}
\omega_{\lambda}^{G}\left(x_{n}, x_{n}, u\right) & \leq \omega_{\frac{\lambda}{2}}^{G}\left(x_{n}, x_{m}, x_{m}\right)+\omega_{\frac{\lambda}{4}}^{G}\left(x_{m}, x_{m}, x_{k}\right)+\omega_{\frac{\lambda}{2}}^{G}\left(x_{k}, x_{k}, u\right) \\
& \leq C_{2} \omega_{\lambda}^{G}\left(x_{n}, x_{m}, x_{m}\right)+C_{4} \omega_{\lambda}^{G}\left(x_{m}, x_{m}, x_{k}\right)+C_{4} \omega_{\lambda}^{G}\left(x_{k}, x_{k}, u\right) \\
& <C_{2} \frac{\epsilon}{3 C_{2}}+C_{4} \frac{\epsilon}{3 C_{4}}+C_{4} \frac{\epsilon}{3 C_{4}} \\
& =\frac{\epsilon}{3}+\frac{\epsilon}{3}+\frac{\epsilon}{3}=\epsilon .
\end{aligned}
$$


Thus $\omega_{\lambda}^{G}\left(x_{n}, x_{n}, u\right)<\epsilon$ for all $n \geq m$ and $\lambda>0$, showing that $\left\{x_{n}\right\}_{n \geq 1}$ converges to $u \in X_{\omega^{G}}$. Hence $\left(X, \omega^{G}\right)$ is a modular $G$-complete modular $G$-metric space.

(ii) $T$ satisfies inequality (3.1) of Theorem 3.1. Indeed, take $|x| \leq \frac{1}{2},|y| \leq \frac{1}{2}$ and $|z| \leq \frac{1}{2}$. By Definition of the modular G-metric, we get

$$
\begin{aligned}
\omega_{\lambda}^{G}(T x, T y, T z) & =\frac{1}{\lambda} \max \{|T x-T y|,|T y-T z|,|T z-T x|\} \quad \forall x, y, z \in X_{\omega^{G}} \\
& =\max \left\{\left|\frac{x^{2}}{2}-\frac{y^{2}}{2}\right|,\left|\frac{y^{2}}{2}-\frac{z^{2}}{2}\right|,\left|\frac{x^{2}}{2}-\frac{z^{2}}{2}\right|\right\} \\
& =\frac{1}{2} \max \left\{\left|x^{2}-y^{2}\right|,\left|y^{2}-z^{2}\right|,\left|x^{2}-z^{2}\right|\right\} \\
& =\frac{1}{2} \max \{|x+y||x-y|,|y+z||y-z|,|x+z||x-z|\} \\
& \leq \frac{1}{2} \max \{(|x|+|y|)|x-y|,(|y|+|z|)|y-z|,(|x|+|z|)|x-z|\} \\
& \leq \frac{1}{2} \max \{|x-y|,|y-z|,|x-z|\} \\
& =\frac{1}{2} \omega_{\lambda}^{G}(x, y, z) \\
& \leq \frac{1}{2} K_{\lambda}(x, y, z),
\end{aligned}
$$

where

$$
K_{\lambda}(x, y, z) \quad\left\{\begin{array}{l}
\omega_{\lambda}^{G}(x, y, z), \omega_{\lambda}^{G}(x, z, T y), \omega_{\lambda}^{G}(T x, y, z), \omega_{\lambda}^{G}(x, T x, y), \omega_{\lambda}^{G}(y, z, T y), \\
\omega_{\lambda}^{G}(x, T x, T x), \omega_{\lambda}^{G}(x, T y, T y), \frac{\omega_{\lambda}^{G}(y, z, T y)\left[1+\omega_{\lambda}^{G}(x, T x, y)\right]}{1+\omega_{\lambda}^{G}(x, y, z)}, \\
\omega_{\lambda}^{G}(x, T x, z), \omega_{\lambda}^{G}(y, T y, T y), \frac{\omega_{\lambda}^{G}(x, T y, z)\left[1+\omega_{\lambda}^{G}(x, T x, y)\right]}{1+\omega_{\lambda}^{G}(x, y, z)} \\
\omega_{\lambda}^{G}(y, T x, T y), \frac{\omega_{\lambda}^{G}(x, T y, z)\left[1+\omega_{\lambda}^{G}(x, y, z)\right]}{1+\omega_{\lambda}^{G}(x, y, z)+\omega_{\lambda}^{G}(y, T x, z)}, \omega_{\lambda}^{G}\left(y, T^{2} x, T y\right), \\
\omega_{\lambda}^{G}(y, T z, T z), \frac{\omega_{\lambda}^{G}(x, T y, z)\left[1+\omega_{\lambda}^{G}(x, y, T y)\right]}{1+\omega_{\lambda}^{G}(T x, y, z)+\omega_{\lambda}^{G}(x, z, T y)}, \omega_{\lambda}^{G}(z, T x, T x), \\
\omega_{\lambda}^{G}(z, T z, T z), \omega_{\lambda}^{G}(z, T x, T y), \omega_{\lambda}^{G}\left(z, T^{2} x, T z\right), \omega_{\lambda}^{G}\left(T x, T^{2} x, T y\right), \\
\omega_{\lambda}^{G}\left(T x, T^{2} x, T z\right), \frac{\omega_{\lambda}^{G}(x, z, T y)\left[1+\omega_{\lambda}^{G}(x, z, T y)\right]}{1+\omega_{\lambda}^{G}(T x, y, z)+\omega_{\lambda}^{G}(x, z, T y)}, \\
\frac{\omega_{\lambda}^{G}\left(T^{2} x, T y, T z\right)\left[1+\omega_{\lambda}^{G}(T x, y, z)+\omega_{\lambda}^{G}(T x, y, T z)\right]}{1+\omega_{\lambda}^{G}(T x, y, T z)+\omega_{\lambda}^{G}\left(T^{2} x, T y, T z\right)}
\end{array}\right\} .
$$

(iii) $T$ has a unique fixed point at $x=0$ and 2 . To see this, we know that $T x=x$ is the fixed point of $T$. Therefore, we have

$$
T x=x \quad \Longrightarrow \quad T x-x=0
$$




$$
\begin{aligned}
& \Longrightarrow \quad \frac{x^{2}}{2}-x=0 \\
& \Longrightarrow \quad x^{2}-2 x=0 \\
& \Longrightarrow \quad x(x-2)=0 \\
& \Longrightarrow \quad x=0 \text { or } x=2 .
\end{aligned}
$$

To check, we get for $x=0, T(0)=\frac{0^{2}}{2}=0$ and for $x=2, T(2)=\frac{2^{2}}{2}=2$.

(iv) $T$ is modular $G$-continuous at the fixed point $u \in X_{\omega^{G}}$. Indeed, to avoid duplication of proof, if we take a sequence $\left\{x_{n}\right\}_{n \geq 1} \subseteq X$ such that $\left\{x_{n}\right\}_{n \geq 1}$ converges to $u=\frac{x^{2}}{2}$, then it follows from inequalities (3.24) to (3.34) that

$$
\omega_{\lambda}^{G}\left(u, T x_{n}, T x_{n}\right) \longrightarrow 0 \quad \forall \lambda>0, \text { as } n \rightarrow \infty .
$$

This shows that $T x_{n}=u=T u$ as $n \rightarrow \infty$.

Example 3.2 Let $X=\mathbb{R} \cup\{\infty\} \subseteq \overline{\mathbb{R}}$. Define $G: \mathbb{R} \times \mathbb{R} \times \mathbb{R} \rightarrow[0, \infty)$ by $G(x, y, z)=\max \{\mid x-$ $y|| y-,z|| x-z \mid$,$\} for all distinct x, y, z \in \mathbb{R}$ and 0 for $x=y=z$. For any $\lambda>0$, let $\omega_{\lambda}^{G}(x, y, z)=$ $\frac{G(x, y, z)}{\lambda}$ for all $x, y, z \in \mathbb{R}$. Define a map $T: \mathbb{R} \rightarrow \mathbb{R}$ by $T x=\frac{\sin ^{2}(x)}{6} \forall x \in \mathbb{R}$.

Indeed, the real line endowed with the above modular $G$-metric is a complete modular $G$-metric space and it follows directly from (i) of Example 3.1 above. $T$ has a trivial fixed point at $x=0$ and $x=\arcsin (6)$, i.e. we know that $T w=w$ is the fixed point of $T$ and take $w=\sin (x)$. Therefore, we have

$$
\begin{aligned}
T w=w & \Longrightarrow T w-w=0 \\
& \Longrightarrow \frac{w^{2}}{6}-w=0 \\
& \Longrightarrow w^{2}-6 w=0 \\
& \Longrightarrow w(w-6)=0 \\
& \Longrightarrow x=0 \quad \text { or } \quad x=\arcsin (6)
\end{aligned}
$$

and it is $G$-continuous at $u \in X_{\omega^{G}}$ say. To see this, if we take a sequence $\left\{x_{n}\right\}_{n \geq 1} \subseteq X$ such that $\left\{x_{n}\right\}_{n \geq 1}$ converges to $u$, then it follows from inequalities (3.24) to (3.34) that

$$
\omega_{\lambda}^{G}\left(u, T x_{n}, T x_{n}\right) \longrightarrow 0 \quad \forall \lambda>0, \text { as } n \rightarrow \infty .
$$

This shows that $T x_{n}=u=T u$ as $n \rightarrow \infty$.

Lastly, for all $x, y, z \in \mathbb{R}$ and taking $|\sin (z)|<|z|$,

$$
\begin{aligned}
G(T x, T y, T z) & =\max \{|T x-T y|,|T y-T z|,|T x-T z|\} \\
& =\frac{1}{6} \max \left\{\left|\sin ^{2}(x)-\sin ^{2}(y)\right|,\left|\sin ^{2}(y)-\sin ^{2}(z)\right|,\left|\sin ^{2}(x)-\sin ^{2}(z)\right|\right\} \\
& =\frac{1}{6} \max \left\{\begin{array}{l}
|\sin (x+y) \sin (x-y)|,|\sin (y+z) \sin (y-z)|, \\
|\sin (x+z) \sin (x-z)|
\end{array}\right\} \\
& \leq \frac{1}{6} \max \{|x-y|,|y-z|,|x-z|\},
\end{aligned}
$$




$$
\omega_{\lambda}^{G}(T x, T y, T z) \leq \frac{1}{6} K_{\lambda}(x, y, z),
$$

where

$$
K_{\lambda}(x, y, z) \quad\left\{\begin{array}{l}
\omega_{\lambda}^{G}(x, y, z), \omega_{\lambda}^{G}(x, z, T y), \omega_{\lambda}^{G}(T x, y, z), \omega_{\lambda}^{G}(x, T x, y), \omega_{\lambda}^{G}(y, z, T y), \\
\omega_{\lambda}^{G}(x, T x, T x), \omega_{\lambda}^{G}(x, T y, T y), \frac{\omega_{\lambda}^{G}(y, z, T y)\left[1+\omega_{\lambda}^{G}(x, T x, y)\right]}{1+\omega_{\lambda}^{G}(x, y, z)} \\
\omega_{\lambda}^{G}(x, T x, z), \omega_{\lambda}^{G}(y, T y, T y), \frac{\omega_{\lambda}^{G}(x, T y, z)\left[1+\omega_{\lambda}^{G}(x, T x, y)\right]}{1+\omega_{\lambda}^{G}(x, y, z)} \\
\omega_{\lambda}^{G}(y, T x, T y), \frac{\omega_{\lambda}^{G}(x, T y, z)\left[1+\omega_{\lambda}^{G}(x, y, z)\right]}{1+\omega_{\lambda}^{G}(x, y, z)+\omega_{\lambda}^{G}(y, T x, z)}, \omega_{\lambda}^{G}\left(y, T^{2} x, T y\right), \\
\omega_{\lambda}^{G}(y, T z, T z), \frac{\omega_{\lambda}^{G}(x, T y, z)\left[1+\omega_{\lambda}^{G}(x, y, T y)\right]}{1+\omega_{\lambda}^{G}(T x, y, z)+\omega_{\lambda}^{G}(x, z, T y)}, \omega_{\lambda}^{G}(z, T x, T x), \\
\omega_{\lambda}^{G}(z, T z, T z), \omega_{\lambda}^{G}(z, T x, T y), \omega_{\lambda}^{G}\left(z, T^{2} x, T z\right), \\
\omega_{\lambda}^{G}\left(T x, T^{2} x, T y\right), \omega_{\lambda}^{G}\left(T x, T^{2} x, T z\right), \frac{\omega_{\lambda}^{G}(x, z, T y)\left[1+\omega_{\lambda}^{G}(x, z, T y)\right]}{1+\omega_{\lambda}^{G}(T x, y, z)+\omega_{\lambda}^{G}(x, z, T y)}, \\
\frac{\omega_{\lambda}^{G}\left(T^{2} x, T y, T z\right)\left[1+\omega_{\lambda}^{G}(T x, y, z)+\omega_{\lambda}^{G}(T x, y, T z)\right]}{1+\omega_{\lambda}^{G}(T x, y, T z)+\omega_{\lambda}^{G}\left(T^{2} x, T y, T z\right)}
\end{array}\right\} .
$$

Therefore, all the conditions of Theorem 3.1 are satisfied.

Remark 3.4 With some $\omega^{G}$ elements in Theorem 3.1, for $\lambda>0$, we have

$$
\begin{aligned}
& \omega_{\lambda}^{G}(T x, T y, T z) \\
& \quad \leq k \max \left\{\begin{array}{l}
\omega_{\lambda}^{G}(x, y, z), \omega_{\lambda}^{G}(x, T x, T x), \omega_{\lambda}^{G}(y, T y, T y), \omega_{\lambda}^{G}(z, T z, T z), \\
\omega_{\lambda}^{G}(x, T y, T y), \omega_{\lambda}^{G}(z, T y, T y), G(z, T x, T x)
\end{array}\right\},
\end{aligned}
$$

where $k \in(0,1)$. Then $T$ has a unique fixed point say $u$ and is modular $G$-continuous at $u$. This is Theorem 2.1 in [47] in the setting of modular G-metric space.

Its variant form in modular G-metric space is of the right form, for any $\lambda>0, m=$ $1,2, \ldots, r$, then we have

$$
\begin{aligned}
& \omega_{\lambda}^{G}\left(T^{m} x, T^{m} y, T^{m} z\right) \\
& \leq k \max \left\{\begin{array}{l}
\omega_{\lambda}^{G}(x, y, z), \omega_{\lambda}^{G}\left(x, T^{m} x, T^{m} x\right), \omega_{\lambda}^{G}\left(y, T^{m} y, T^{m} y\right), \\
\omega_{\lambda}^{G}\left(z, T^{m} z, T^{m} z\right), \omega_{\lambda}^{G}\left(x, T^{m} y, T^{m} y\right), \\
\omega_{\lambda}^{G}\left(z, T^{m} y, T^{m} y\right), G\left(z, T^{m} x, T^{m} x\right)
\end{array}\right\},
\end{aligned}
$$

where $k \in(0,1)$. Then $T$ has a unique fixed point (say $u$ ) and is modular $G$-continuous at $u$. This is Corollary 2.3 in [47] in the setting of modular G-metric space. 
Remark 3.5 If the statement of Theorem 3.1 holds and

$$
k \max \left\{\begin{array}{l}
\omega_{\lambda}^{G}(x, y, z), \omega_{\lambda}^{G}(x, z, T y), \omega_{\lambda}^{G}(T x, y, z), \omega_{\lambda}^{G}(x, T x, y), \omega_{\lambda}^{G}(y, z, T y), \omega_{\lambda}^{G}(x, T x, T x), \\
\omega_{\lambda}^{G}(x, T y, T y), \frac{\omega_{\lambda}^{G}(y, z, T y)\left[1+\omega_{\lambda}^{G}(x, T x, y)\right]}{1+\omega_{\lambda}^{G}(x, y, z)}, \omega_{\lambda}^{G}(x, T x, z), \omega_{\lambda}^{G}(y, T y, T y), \\
\frac{\omega_{\lambda}^{G}(x, T y, z)\left[1+\omega_{\lambda}^{G}(x, T x, y)\right]}{1+\omega_{\lambda}^{G}(x, y, z)}, \omega_{\lambda}^{G}(y, T x, T y), \frac{\omega_{\lambda}^{G}(x, T y, z)\left[1+\omega_{\lambda}^{G}(x, y, z)\right]}{1+\omega_{\lambda}^{G}(x, y, z)+\omega_{\lambda}^{G}(y, T x, z)}, \\
\omega_{\lambda}^{G}(z, T z, T z), \omega_{\lambda}^{G}(z, T x, T y), \omega_{\lambda}^{G}\left(z, T^{2} x, T z\right), \\
\omega_{\lambda}^{G}(T x, T y), \omega_{\lambda}^{G}(y, T z, T z), \frac{\omega_{\lambda}^{G}(x, T y, z)\left[1+\omega_{\lambda}^{G}(x, y, T y)\right]}{1+\omega_{\lambda}^{G}(T x, y, z)+\omega_{\lambda}^{G}(x, z, T y)}, \omega_{\lambda}^{G}(z, T x, T x), \\
\frac{\omega_{\lambda}^{G}\left(T^{2} x, T y, T z\right)\left[1+\omega_{\lambda}^{G}(T x, y, z)+\omega_{\lambda}^{G}(T x, y, T z)\right]}{1+\omega_{\lambda}^{G}(T x, y, T z)+\omega_{\lambda}^{G}\left(T^{2} x, T y, T z\right)} \\
=k\left\{\omega_{\lambda}^{G}(x, T x, T x)+\omega_{\lambda}^{G}(y, T y, T y)+\omega_{\lambda}^{G}(z, T z, T z)\right\}
\end{array}\right\}
$$

for all $x, y, z \in M \subseteq X_{\omega^{G}}$. This is Theorem 3.6 of [14].

Corollary 3.2 Let $\left(X, \omega^{G}\right)$ be a G-complete non-symmetric modular G-metric space satisfying a $\Delta_{3}$-type condition, such that $C_{2} C_{4} \in\left(0, \frac{1}{4 \rho}\right)$ and let $T: X_{\omega^{G}} \rightarrow X_{\omega^{G}}$ be a mapping. Suppose that there exists $\lambda>0$, and $k \in(0,1)$ such that

$\omega_{\lambda}^{G}(T x, T y, T z)$

$$
\begin{aligned}
& \leq k \max \left\{\begin{array}{l}
\omega_{\lambda}^{G}(x, y, z), \omega_{\lambda}^{G}(x, z, T y), \omega_{\lambda}^{G}(T x, y, z), \omega_{\lambda}^{G}(x, T x, y), \omega_{\lambda}^{G}(y, z, T y), \\
\omega_{\lambda}^{G}(x, T x, T x), \omega_{\lambda}^{G}(x, T y, T y), \frac{\omega_{\lambda}^{G}(y, z, T y)\left[1+\omega_{\lambda}^{G}(x, T x, y)\right]}{1+\omega_{\lambda}^{G}(x, y, z)} \\
\omega_{\lambda}^{G}(x, T x, z), \omega_{\lambda}^{G}(y, T y, T y), \frac{\omega_{\lambda}^{G}(x, T y, z)\left[1+\omega_{\lambda}^{G}(x, T x, y)\right]}{1+\omega_{\lambda}^{G}(x, y, z)} \\
\omega_{\lambda}^{G}(y, T x, T y), \frac{\omega_{\lambda}^{G}(x, T y, z)\left[1+\omega_{\lambda}^{G}(x, y, z)\right]}{1+\omega_{\lambda}^{G}(x, y, z)+\omega_{\lambda}^{G}(y, T x, z)}, \omega_{\lambda}^{G}\left(y, T^{2} x, T y\right), \\
\omega_{\lambda}^{G}(y, T z, T z), \frac{\omega_{\lambda}^{G}(x, T y, z)\left[1+\omega_{\lambda}^{G}(x, y, T y)\right]}{1+\omega_{\lambda}^{G}(T x, y, z)+\omega_{\lambda}^{G}(x, z, T y)}, \omega_{\lambda}^{G}(z, T x, T x), \\
\omega_{\lambda}^{G}(z, T z, T z), \omega_{\lambda}^{G}(z, T x, T y), \omega_{\lambda}^{G}\left(z, T^{2} x, T z\right), \\
\omega_{\lambda}^{G}\left(T x, T^{2} x, T y\right), \omega_{\lambda}^{G}\left(T x, T^{2} x, T z\right), \frac{\omega_{\lambda}^{G}(x, z, T y)\left[1+\omega_{\lambda}^{G}(x, z, T y)\right]}{1+\omega_{\lambda}^{G}(T x, y, z)+\omega_{\lambda}^{G}(x, z, T y)} \\
\frac{\omega_{\lambda}^{G}\left(T^{2} x, T y, T z\right)\left[1+\omega_{\lambda}^{G}(T x, y, z)+\omega_{\lambda}^{G}(T x, y, T z)\right]}{1+\omega_{\lambda}^{G}(T x, y, T z)+\omega_{\lambda}^{G}\left(T^{2} x, T y, T z\right)}
\end{array}\right\} \\
& =k\left\{\begin{array}{c}
\omega_{\lambda}^{G}(x, y, z)+\omega_{\lambda}^{G}(x, T x, T x)+\omega_{\lambda}^{G}(y, T y, T y)+\omega_{\lambda}^{G}(z, T z, T z) \\
+\omega_{\lambda}^{G}(T x, y, z)+\omega_{\lambda}^{G}\left(y, T^{2} x, T y\right)+\omega_{\lambda}^{G}(y, T z, T z)+\omega_{\lambda}^{G}(z, T x, T x) \\
+\omega_{\lambda}^{G}\left(z, T^{2} x, T z\right)+\omega_{\lambda}^{G}\left(T x, T^{2} x, T y\right)+\omega_{\lambda}^{G}\left(T x, T^{2} x, T z\right)
\end{array}\right\}
\end{aligned}
$$


for all $x, y, z \in M \subseteq X_{\omega^{G}}, \lambda>0$. Then $T$ has a unique fixed point in $X_{\omega^{G}}$ and is modular G-continuous at the fixed point (say $u)$.

Proposition 3.3 Let $\left(X, \omega^{G}\right)$ be a G-complete non-symmetric modular G-metric space satisfying a $\Delta_{3}$-type condition, $C_{2} C_{4} \in\left(0, \frac{1}{4 \rho}\right)$ and let $T: X_{\omega^{G}} \rightarrow X_{\omega^{G}}$ be a mapping. Suppose that there exists $\lambda>0$, and $\rho \in(0,1)$ such that

$$
\begin{aligned}
& \omega_{\lambda}^{G}(T x, T y, T z) \\
& \quad \leq \rho \max \left\{\begin{array}{l}
\omega_{\lambda}^{G}(T x, y, z), \omega_{\lambda}^{G}(z, T x, T x), \\
\frac{\omega_{\lambda}^{G}\left(T^{2} x, T y, T z\right)\left[1+\omega_{\lambda}^{G}(T x, y, z)+\omega_{\lambda}^{G}(T x, y, T z)\right]}{1+\omega_{\lambda}^{G}(T x, y, T z)+\omega_{\lambda}^{G}\left(T^{2} x, T y, T z\right)}
\end{array}\right\}
\end{aligned}
$$

for all $x, y, z \in M \subseteq X_{\omega^{G}}, \lambda>0$. Then $T$ has a unique fixed point in $X_{\omega^{G}}$ and is modular G-continuous at the fixed point (say $u$ ).

Proof Let $x_{0} \in X_{\omega^{G}}$ be arbitrary. We generate the sequence of iteration of $T$ based on $x_{0} \in X_{\omega^{G}}$ as follows:

$$
\begin{gathered}
T x_{0}=x_{1} \\
T x_{1}=x_{2} \\
\vdots \\
T x_{n}=x_{n+1}
\end{gathered}
$$

for all $n \in \mathbb{N}$. If there exists some $n_{0} \in \mathbb{N}$ such that $x_{n_{0}+1}=x_{n_{0}}$ then $x_{0}$ is a fixed point of $T$. Now for all $n \in \mathbb{N}, x_{n+1} \neq x_{n}$, so that $\omega_{\lambda}^{G}\left(n_{n}, x_{n+1}, x_{n+1}\right)>0$ and for $\lambda>0$, take $x=x_{n}$ and $y=x_{n+1}=z$, then we have $\omega_{\lambda}^{G}\left(x_{n+1}, x_{n+2}, x_{n+2}\right)=\omega_{\lambda}^{G}(T x, T y, T z)=\omega_{\lambda}^{G}\left(T x_{n}, T x_{n+1}, T x_{n+1}\right)$ so that $\forall x_{n}, x_{n+1} \in X_{\omega^{G}}, \lambda>0$, inequality (3.49) becomes

$$
\begin{aligned}
& \omega_{\lambda}^{G}\left(x_{n+1}, x_{n+2}, x_{n+2}\right) \\
& \leq \rho \max \left\{\begin{array}{l}
\omega_{\lambda}^{G}\left(T x_{n}, x_{n+1}, x_{n+1}\right), \omega_{\lambda}^{G}\left(x_{n+1}, T x_{n}, T x_{n}\right), \\
\frac{\omega_{\lambda}^{G}\left(T^{2} x_{n}, T x_{n+1}, T x_{n+1}\right)\left[1+\omega_{\lambda}^{G}\left(T x_{n}, x_{n+1}, x_{n+1}\right)+\omega_{\lambda}^{G}\left(T x_{n}, x_{n+1}, T x_{n+1}\right)\right]}{1+\omega_{\lambda}^{G}\left(T x_{n}, x_{n+1}, T x_{n+1}\right)+\omega_{\lambda}^{G}\left(T^{2} x_{n}, T x_{n+1}, T x_{n+1}\right)}
\end{array}\right\} \\
& =\rho \max \left\{\begin{array}{l}
\omega_{\lambda}^{G}\left(x_{n+1}, x_{n+1}, x_{n+1}\right), \omega_{\lambda}^{G}\left(x_{n+1}, x_{n+1}, x_{n+1}\right), \\
\frac{\omega_{\lambda}^{G}\left(x_{n+2}, x_{n+2}, x_{n+2}\right)\left[1+\omega_{\lambda}^{G}\left(x_{n+1}, x_{n+1}, x_{n+1}\right)+\omega_{\lambda}^{G}\left(x_{n+1}, x_{n+1}, x_{n+2}\right)\right]}{1+\omega_{\lambda}^{G}\left(x_{n+1}, x_{n+1}, x_{n+2}\right)+\omega_{\lambda}^{G}\left(x_{n+2}, x_{n+2}, x_{n+2}\right)}
\end{array}\right\} .
\end{aligned}
$$

Therefore, we have $\omega_{\lambda}^{G}\left(x_{n+1}, x_{n+2}, x_{n+2}\right) \leq 0$. For $m>n \in \mathbb{N}$, we get

$$
\lim _{n, m \rightarrow \infty} \omega_{\lambda}^{G}\left(x_{n}, x_{m}, x_{m}\right)=0
$$

For $n, m, k \in \mathbb{N}$, condition (2) of Proposition 2.2 implies that

$$
\omega_{\lambda}^{G}\left(x_{n}, x_{m}, x_{k}\right) \leq \omega_{\frac{\lambda}{2}}^{G}\left(x_{n}, x_{m}, x_{m}\right)+\omega_{\frac{\lambda}{2}}^{G}\left(x_{k}, x_{m}, x_{m}\right)
$$


so that on taking the limit of both sides of inequality (3.52) as $n, m, l \rightarrow \infty$ and by applying Definition 2.11 and Eq. (3.51), we get

$$
\begin{aligned}
\lim _{n, m, k \rightarrow \infty} \omega_{\lambda}^{G}\left(x_{n}, x_{m}, x_{k}\right) & \leq \lim _{n, m \rightarrow \infty} \omega_{\frac{\lambda}{2}}^{G}\left(x_{n}, x_{m}, x_{m}\right)+\lim _{k, m \rightarrow \infty} \omega_{\frac{\lambda}{2}}^{G}\left(x_{k}, x_{m}, x_{m}\right) \\
& \leq C_{2} \lim _{n, m \rightarrow \infty} \omega_{\lambda}^{G}\left(x_{n}, x_{m}, x_{m}\right)+C_{2} \lim _{k, m \rightarrow \infty} \omega_{\lambda}^{G}\left(x_{k}, x_{m}, x_{m}\right) \\
& =C_{2}\left(\lim _{n, m \rightarrow \infty} \omega_{\lambda}^{G}\left(x_{n}, x_{m}, x_{m}\right)+\lim _{k, m \rightarrow \infty} \omega_{\lambda}^{G}\left(x_{k}, x_{m}, x_{m}\right)\right),
\end{aligned}
$$

thus we have

$$
\lim _{n, m, k \rightarrow \infty} \omega_{\lambda}^{G}\left(x_{n}, x_{m}, x_{k}\right)=0
$$

Equation (3.54) shows that $\left\{x_{n}\right\}_{n \in \mathbb{N}}$ is a modular G-Cauchy sequence. By Theorem 3.1, $T$ has a unique fixed point in $X_{\omega^{G}}$ and also is modular $G$-continuous at the fixed point $u$.

Proposition 3.4 Let $\left(X, \omega^{G}\right)$ be a G-complete non-symmetric modular G-metric space satisfying a $\Delta_{3}$-type condition, such that $C_{2} C_{4} \in\left(0, \frac{1}{4 \rho}\right)$ and let $T: X_{\omega^{G}} \rightarrow X_{\omega^{G}}$ be a mapping. Suppose that there exists $\lambda>0$, and $\rho \in(0,1)$ such that

$$
\begin{aligned}
& \omega_{\lambda}^{G}(T x, T y, T z) \\
& \quad \leq \rho \max \left\{\begin{array}{l}
\omega_{\lambda}^{G}(x, y, z), \omega_{\lambda}^{G}(T x, y, z), \omega_{\lambda}^{G}(z, T x, T x), \\
\frac{\omega_{\lambda}^{G}\left(T^{2} x, T y, T z\right)\left[1+\omega_{\lambda}^{G}(T x, y, z)+\omega_{\lambda}^{G}(T x, y, T z)\right]}{1+\omega_{\lambda}^{G}(T x, y, T z)+\omega_{\lambda}^{G}\left(T^{2} x, T y, T z\right)}
\end{array}\right\} .
\end{aligned}
$$

Then $T$ has a unique fixed point in $X_{\omega^{G}}$ and is modular G-continuous at the fixed point (say u).

Proof From Proposition 3.3, $\omega_{\lambda}^{G}\left(x_{n+1}, x_{n+2}, x_{n+2}\right) \leq \rho \omega_{\lambda}^{G}\left(x_{n}, x_{n+1}, x_{n+1}\right)$, so that

$$
\omega_{\lambda}^{G}\left(x_{n+1}, x_{n+2}, x_{n+2}\right) \leq \rho \omega_{\lambda}^{G}\left(x_{n}, x_{n+1}, x_{n+1}\right),
$$

therefore,

$$
\begin{aligned}
\omega_{\lambda}^{G}\left(x_{n+1}, x_{n+2}, x_{n+2}\right) & \leq \rho \omega_{\lambda}^{G}\left(x_{n}, x_{n+1}, x_{n+1}\right) \\
& \leq \rho^{2} \omega_{\lambda}^{G}\left(x_{n-1}, x_{n}, x_{n}\right) \\
& \leq \rho^{3} \omega_{\lambda}^{G}\left(x_{n-2}, x_{n-1}, x_{n-1}\right) \\
& \vdots \\
& \leq \rho^{n} \omega_{\lambda}^{G}\left(x_{0}, x_{1}, x_{1}\right) .
\end{aligned}
$$

But $\sum_{n \in \mathbb{N}} \rho^{n}<+\infty$. Now $\sum_{n \in \mathbb{N}} \omega_{\lambda}^{G}\left(x_{n+1}, x_{n+2}, x_{n+2}\right) \leq \omega_{\lambda}^{G}\left(x_{0}, x_{1}, x_{1}\right) \sum_{n \in \mathbb{N}} \rho^{n}<+\infty$ for all $\lambda>0$. Suppose that $m, n \in \mathbb{N}$ and $m>n \in \mathbb{N}$. Observe that, for any arbitrary $\epsilon$, using the 
rectangle inequality repeatedly and condition (2) of Proposition 2.2, we have

$$
\begin{aligned}
\omega_{\lambda}^{G}\left(x_{n}, x_{m}, x_{m}\right) \leq & \omega_{\frac{\lambda}{m-n}}^{G}\left(x_{n}, x_{n+1}, x_{n+1}\right)+\omega_{\frac{\lambda}{m-n}}^{G}\left(x_{n+1}, x_{n+2}, x_{n+2}\right)+\omega_{\frac{\lambda}{m-n}}^{G}\left(x_{n+2}, x_{n+3}, x_{n+3}\right) \\
& +\omega_{\frac{\lambda}{m-n}}^{G}\left(x_{n+3}, x_{n+4}, x_{n+4}\right)+\cdots+\omega_{\frac{\lambda}{m-n}}^{G}\left(x_{m-1}, x_{m}, x_{m}\right) \\
\leq & \omega_{\frac{\lambda}{m}}^{G}\left(x_{n}, x_{n+1}, x_{n+1}\right)+\omega_{\frac{\lambda}{m}}^{G}\left(x_{n+1}, x_{n+2}, x_{n+2}\right)+\omega_{\frac{\lambda}{m}}^{G}\left(x_{n+2}, x_{n+3}, x_{n+3}\right) \\
& +\omega_{\frac{\lambda}{m}}^{G}\left(x_{n+3}, x_{n+4}, x_{n+4}\right)+\cdots+\omega_{\frac{\lambda}{m}}^{G}\left(x_{m-1}, x_{m}, x_{m}\right) \\
\leq & \sum_{n=N} \omega_{\lambda}^{G}\left(x_{n+1}, x_{n+2}, x_{n+2}\right) \\
< & \epsilon
\end{aligned}
$$

for all $m>n \geq N$ for some $N \in \mathbb{N}$. As $\epsilon$ is arbitrary, we have

$$
\omega_{\lambda}^{G}\left(x_{n}, x_{m}, x_{m}\right)=0 \quad \text { as } n, m \rightarrow \infty \quad \text { or } \quad \lim _{n, m \rightarrow \infty} \omega_{\lambda}^{G}\left(x_{n}, x_{m}, x_{m}\right)=0
$$

For $n, m, k \in \mathbb{N}$, condition (2) of Proposition 2.2 implies that

$$
\omega_{\lambda}^{G}\left(x_{n}, x_{m}, x_{k}\right) \leq \omega_{\frac{\lambda}{2}}^{G}\left(x_{n}, x_{m}, x_{m}\right)+\omega_{\frac{\lambda}{2}}^{G}\left(x_{k}, x_{m}, x_{m}\right),
$$

so that on taking the limit of both sides of inequality (3.60) as $n, m, l \rightarrow \infty$ and by applying Definition 2.11 and Eq. (3.59), we get

$$
\begin{aligned}
\lim _{n, m, k \rightarrow \infty} \omega_{\lambda}^{G}\left(x_{n}, x_{m}, x_{k}\right) & \leq \lim _{n, m \rightarrow \infty} \omega_{\frac{\lambda}{2}}^{G}\left(x_{n}, x_{m}, x_{m}\right)+\lim _{k, m \rightarrow \infty} \omega_{\frac{\lambda}{2}}^{G}\left(x_{k}, x_{m}, x_{m}\right) \\
& \leq C_{2} \lim _{n, m \rightarrow \infty} \omega_{\lambda}^{G}\left(x_{n}, x_{m}, x_{m}\right)+C_{2} \lim _{k, m \rightarrow \infty} \omega_{\lambda}^{G}\left(x_{k}, x_{m}, x_{m}\right) \\
& =C_{2}\left(\lim _{n, m \rightarrow \infty} \omega_{\lambda}^{G}\left(x_{n}, x_{m}, x_{m}\right)+\lim _{k, m \rightarrow \infty} \omega_{\lambda}^{G}\left(x_{k}, x_{m}, x_{m}\right)\right)
\end{aligned}
$$

thus we have

$$
\lim _{n, m, k \rightarrow \infty} \omega_{\lambda}^{G}\left(x_{n}, x_{m}, x_{k}\right)=0
$$

Equation (3.62) shows that $\left\{x_{n}\right\}_{n \in \mathbb{N}}$ is a modular G-Cauchy sequence. By Theorem 3.1, $T$ has a unique fixed point in $X_{\omega^{G}}$ and is modular $G$-continuous at the fixed point $u$.

Proposition 3.5 Let $\left(X, \omega^{G}\right)$ be a G-complete non-symmetric modular G-metric space satisfying a $\Delta_{3}$-type condition, such that $C_{2} C_{4} \in\left(0, \frac{1}{4 \rho}\right)$ and let $T: X_{\omega^{G}} \rightarrow X_{\omega^{G}}$ be a mapping. Suppose that there exists some positive integer $m \geq 1, \lambda>0$, and $\rho \in(0,1)$ such that

$$
\begin{aligned}
& \omega_{\lambda}^{G}\left(T^{m} x, T^{m} y, T^{m} z\right) \\
& \quad \leq \rho \max \left\{\begin{array}{l}
\omega_{\lambda}^{G}\left(T^{m} x, y, z\right), \omega_{\lambda}^{G}\left(z, T^{m} x, T^{m} x\right), \\
\frac{\omega_{\lambda}^{G}\left(T^{m} x, T^{m} y, T^{m} z\right)\left[1+\omega_{\lambda}^{G}\left(T^{m} x, y, z\right)+\omega_{\lambda}^{G}\left(T^{m} x, y, T^{m} z\right)\right]}{1+\omega_{\lambda}^{G}\left(T^{m} x, y, T^{m} z\right)+\omega_{\lambda}^{G}\left(T^{m} x, T^{m} y, T^{m} z\right)}
\end{array}\right\} .
\end{aligned}
$$


Then $T$ has a unique fixed point in $X_{\omega^{G}}$ and is modular G-continuous at the fixed point (say $u$ ) for some positive integer $m \geq 1$.

Proof For $m=2$, we have

$$
\begin{aligned}
& \omega_{\lambda}^{G}\left(T^{2} x, T^{2} y, T^{2} z\right) \\
& \quad \leq \rho \max \left\{\begin{array}{l}
\omega_{\lambda}^{G}\left(T^{2} x, y, z\right), \omega_{\lambda}^{G}\left(z, T^{2} x, T^{2} x\right), \\
\frac{\omega_{\lambda}^{G}\left(T^{2} x, T^{2} y, T^{2} z\right)\left[1+\omega_{\lambda}^{G}\left(T^{2} x, y, z\right)+\omega_{\lambda}^{G}\left(T^{2} x, y, T^{2} z\right)\right]}{1+\omega_{\lambda}^{G}\left(T^{2} x, y, T^{2} z\right)+\omega_{\lambda}^{G}\left(T^{2} x, T^{2} y, T^{2} z\right)}
\end{array}\right\} .
\end{aligned}
$$

Let $x_{0} \in X_{\omega^{G}}$ be arbitrary. We generate the sequence of iteration of $T$ based on $x_{0} \in X_{\omega^{G}}$ as follows:

$$
\begin{aligned}
& T x_{0}=x_{1} \\
& T x_{1}=x_{2} \\
& \vdots \\
& T x_{n}=x_{n+1}
\end{aligned}
$$

for all $n \in \mathbb{N}$. If there exists some $n_{0} \in \mathbb{N}$ such that $x_{n_{0}+1}=x_{n_{0}}$ then $x_{0}$ is a fixed point of $T$. Now for all $n \in \mathbb{N}, x_{n+1} \neq x_{n}$ and $\lambda>0$, take $x=x_{n}$ and $y=x_{n+1}=z$, then we have $\omega_{\lambda}^{G}\left(x_{n+2}, x_{n+3}, x_{n+3}\right)=\omega_{\lambda}^{G}\left(T^{2} x, T^{2} y, T^{2} z\right)=\omega_{\lambda}^{G}\left(T^{2} x_{n}, T^{2} x_{n+1}, T^{2} x_{n+1}\right)$ so that $\forall x_{n}, x_{n+1} \in X_{\omega}$, $\lambda>0$, inequality (3.64) becomes

$$
\begin{aligned}
& \omega_{\lambda}^{G}\left(x_{n+2}, x_{n+3}, x_{n+3}\right) \\
& \quad \leq \rho \max \left\{\begin{array}{l}
\omega_{\lambda}^{G}\left(T^{2} x_{n}, x_{n+1}, x_{n+1}\right), \omega_{\lambda}^{G}\left(x_{n+1}, T^{2} x_{n}, T^{2} x_{n}\right), \\
\frac{\omega_{\lambda}^{G}\left(T^{2} x_{n}, T^{2} x_{n+1}, T^{2} x_{n+1}\right)\left[1+\omega_{\lambda}^{G}\left(T^{2} x_{n}, x_{n+1}, x_{n+1}\right)+\omega_{\lambda}^{G}\left(T^{2} x_{n}, x_{n+1}, T^{2} x_{n+1}\right)\right]}{1+\omega_{\lambda}^{G}\left(T^{2} x_{n}, x_{n+1}, T^{2} x_{n+1}\right)+\omega_{\lambda}^{G}\left(T^{2} x_{n}, T^{2} x_{n+1}, T^{2} x_{n+1}\right)}
\end{array}\right\} .
\end{aligned}
$$

So,

$$
=\rho \max \left\{\begin{array}{l}
\omega_{\lambda}^{G}\left(x_{n+2}, x_{n+1}, x_{n+1}\right), \omega_{\lambda}^{G}\left(x_{n+1}, x_{n+2}, x_{n+2}\right), \\
\frac{\omega_{\lambda}^{G}\left(x_{n+2}, x_{n+3}, x_{n+3}\right)\left[1+\omega_{\lambda}^{G}\left(x_{n+2}, x_{n+1}, x_{n+1}\right)+\omega_{\lambda}^{G}\left(x_{n+2}, x_{n+1}, x_{n+3}\right)\right]}{1+\omega_{\lambda}^{G}\left(x_{n+2}, x_{n+1}, x_{n+3}\right)+\omega_{\lambda}^{G}\left(x_{n+2}, x_{n+3}, x_{n+3}\right)}
\end{array}\right\} .
$$

Proposition 3.4 shows that $T$ has a unique fixed point in $X_{\omega^{G}}$. For $m=3,4, \ldots, k, T^{m} u$ has a unique fixed point $u$ say, that is $T^{m} u=u$. Now we can see that $T u=T\left(T^{m} u\right)=T^{m+1} u=$ $T^{m}(T u)$. Suppose that $T u$ is another fixed point for $T^{m} u$ and by uniqueness of the limit, $T u=u$. By Theorem 3.1, $T^{m} u$ is modular G-continuous at say $u$, i.e. $T^{m} x_{n}=u=T^{m} u$ for some positive integer $m \geq 1$.

Corollary 3.6 Let $\left(X, \omega^{G}\right)$ be a G-complete non-symmetric modular G-metric space satisfying a $\Delta_{3}$-type condition, such that $C_{2} C_{4} \in\left(0, \frac{1}{4 \rho}\right)$ and let $T: X_{\omega^{G}} \rightarrow X_{\omega^{G}}$ be a mapping. Suppose that there exists some positive integer $m \geq 1, \lambda>0$, and $\rho \in(0,1)$ such that

$$
\omega_{\lambda}^{G}\left(T^{m} x, T^{m} y, T^{m} z\right) \leq \rho K_{\lambda}(x, y, z) \quad \text { for all } x, y, z \in X_{\omega^{G}}, \lambda>0,
$$


where

$$
\left.\begin{array}{l}
K_{\lambda}(x, y, z) \\
=\max \left\{\begin{array}{l}
\omega_{\lambda}^{G}(x, y, z), \omega_{\lambda}^{G}\left(x, z, T^{m} y\right), \omega_{\lambda}^{G}\left(T^{m} x, y, z\right), \omega_{\lambda}^{G}\left(x, T^{m} x, y\right), \\
\omega_{\lambda}^{G}\left(y, z, T^{m} y\right), \omega_{\lambda}^{G}\left(x, T^{m} x, T^{m} x\right), \omega_{\lambda}^{G}\left(x, T^{m} y, T^{m} y\right), \\
\frac{\omega_{\lambda}^{G}\left(y, z, T^{m} y\right)\left[1+\omega_{\lambda}^{G}\left(x, T^{m} x, y\right)\right]}{1+\omega_{\lambda}^{G}(x, y, z)}, \omega_{\lambda}^{G}\left(x, T^{m} x, z\right), \omega_{\lambda}^{G}\left(y, T^{m} y, T^{m} y\right), \\
\frac{\omega_{\lambda}^{G}\left(x, T^{m} y, z\right)\left[1+\omega_{\lambda}^{G}\left(x, T^{m} x, y\right)\right]}{1+\omega_{\lambda}^{G}(x, y, z)}, \omega_{\lambda}^{G}\left(y, T^{m} x, T^{m} y\right), \\
\omega_{\lambda}^{G}\left(x, T^{m} y, z\right)\left[1+\omega_{\lambda}^{G}(x, y, z)\right] \\
1+\omega_{\lambda}^{G}(x, y, z)+\omega_{\lambda}^{G}\left(y, T^{m} x, z\right) \\
\omega_{\lambda}^{G}\left(y, T^{m} x, T^{m} y\right), \\
\omega_{\lambda}^{G}\left(z, T^{m} x, T^{m} x\right), \omega_{\lambda}^{G}\left(z, T^{m} z, T^{m} z\right), \omega_{\lambda}^{G}\left(z, T^{m} x, T^{m} y\right), \\
\omega_{\lambda}^{G}\left(z, T^{m} x, T^{m} z\right), \omega_{\lambda}^{G}\left(T^{m} x, T^{m} x, T^{m} y\right), \omega_{\lambda}^{G}\left(T^{m} x, T^{m} x, T^{m} z\right), \\
\frac{\omega_{\lambda}^{G}\left(x, z, T^{m} y\right)\left[1+\omega_{\lambda}^{G}\left(x, z, T^{m} y\right)\right]}{1+\omega_{\lambda}^{G}\left(T^{m} x, y, z\right)+\omega_{\lambda}^{G}\left(x, z, T^{m} y\right)} \\
\omega_{\lambda}^{G}\left(T^{m} x, T^{m} y, T^{m} z\right)\left[1+\omega_{\lambda}^{G}\left(T^{m} x, y, z\right)+\omega_{\lambda}^{G}\left(T^{m} x, y, T^{m} z\right)\right] \\
1+\omega_{\lambda}^{G}\left(T^{m} x, y, T^{m} z\right)+\omega_{\lambda}^{G}\left(T^{m} x, T^{m} y, T^{m} z\right)
\end{array}\right.
\end{array}\right\} .
$$

Then $T$ has a unique fixed point in $X_{\omega^{G}}$ and $T^{m}$ is modular G-continuous at the fixed point (say $u$ ) for some positive integer $m \geq 1$.

Proof $T^{m} u$ has a unique fixed point $u$ say, that is $T^{m} u=u$. Now we see that $T u=T\left(T^{m} u\right)=$ $T^{m+1} u=T^{m}(T u)$. But $T u$ is another fixed point for $T^{m} u$ and by uniqueness of the limit, $T u=u$. By Theorem 3.1, $T^{m} u$ is modular G-continuous at say $u$, i.e. $T^{m} x_{n}=u=T^{m} u$.

Corollary 3.7 Let $\left(X, \omega^{G}\right)$ be a G-complete modular G-metric space satisfying a $\Delta_{3}$-type condition, such that $C_{2} C_{4} \in\left(0, \frac{1}{4 \rho}\right)$ and let $T: X_{\omega^{G}} \rightarrow X_{\omega^{G}}$ be a mapping. Suppose that there exists some positive integer $m \geq 1, \lambda>0$, and $\rho \in(0,1)$ such that

$$
\omega_{\lambda}^{G}\left(T^{m} x, T^{m} y, T^{m} z\right) \leq \rho \omega_{\lambda}^{G}(x, y, z) \quad \text { for all } x, y, z \in X_{\omega^{G}}, \lambda>0
$$

then $T$ has a unique fixed point in $X_{\omega^{G}}$ and $T^{m}$ is modular G-continuous at the fixed point (say $u$ ) for some positive integer $m \geq 1$.

Proof Following Corollary 3.6, $T^{m} u$ has a unique fixed point $u$ say, that is $T^{m} u=u$. Now we see that $T u=T\left(T^{m} u\right)=T^{m+1} u=T^{m}(T u)$. But $T u$ is another fixed point for $T^{m} u$ and by uniqueness of the limit, $T u=u$. By Theorem 3.1, $T^{m} u$ is modular G-continuous at say $u$, i.e. $T^{m} x_{n}=u=T^{m} u$.

Remark 3.6 The variant of Remark 3.5 above reads: let $\left(X, \omega^{G}\right)$ be a $G$-complete nonsymmetric modular $G$-metric space satisfying a $\Delta_{3}$-type condition, such that $C_{2} C_{4} \in$ 
$\left(0, \frac{1}{4 \rho}\right)$ and let $T: X_{\omega^{G}} \rightarrow X_{\omega^{G}}$ be a mapping. Suppose that there exists some positive integer $m \geq 1, \lambda>0$, and $k \in(0,1)$ such that

$$
\omega_{\lambda}^{G}\left(T^{m} x, T^{m} y, T^{m} z\right) \leq k Q_{\lambda}(x, y, z) \quad \text { for all } x, y, z \in X_{\omega^{G}}, \lambda>0,
$$

where

$Q_{\lambda}(x, y, z)$

$$
=k \max \left\{\begin{array}{l}
\omega_{\lambda}^{G}(x, y, z), \omega_{\lambda}^{G}\left(x, z, T^{m} y\right), \omega_{\lambda}^{G}\left(T^{m} x, y, z\right), \omega_{\lambda}^{G}\left(x, T^{m} x, y\right), \omega_{\lambda}^{G}\left(y, z, T^{m} y\right), \\
\omega_{\lambda}^{G}\left(x, T^{m} x, T^{m} x\right), \omega_{\lambda}^{G}\left(x, T^{m} y, T^{m} y\right), \frac{\omega_{\lambda}^{G}\left(y, z, T^{m} y\right)\left[1+\omega_{\lambda}^{G}\left(x, T^{m} x, y\right)\right]}{1+\omega_{\lambda}^{G}(x, y, z)}, \\
\omega_{\lambda}^{G}\left(x, T^{m} x, z\right), \omega_{\lambda}^{G}\left(y, T^{m} y, T^{m} y\right), \frac{\omega_{\lambda}^{G}\left(x, T^{m} y, z\right)\left[1+\omega_{\lambda}^{G}\left(x, T^{m} x, y\right)\right]}{1+\omega_{\lambda}^{G}(x, y, z)}, \\
\omega_{\lambda}^{G}\left(y, T^{m} x, T^{m} y\right), \omega_{\lambda}^{G}\left(y, T^{m} x, T^{m} y\right), \frac{\omega_{\lambda}^{G}\left(x, T^{m} y, z\right)\left[1+\omega_{\lambda}^{G}(x, y, z)\right]}{1+\omega_{\lambda}^{G}(x, y, z)+\omega_{\lambda}^{G}\left(y, T^{m} x, z\right)}, \\
\omega_{\lambda}^{G}\left(y, T^{m} z, T^{m} z\right), \frac{\omega_{\lambda}^{G}(x, T y, z)\left[1+\omega_{\lambda}^{G}\left(x, y, T^{m} y\right)\right]}{1+\omega_{\lambda}^{G}\left(T^{m} x, y, z\right)+\omega_{\lambda}^{G}\left(x, z, T^{m} y\right)}, \\
\omega_{\lambda}^{G}\left(z, T^{m} x, T^{m} x\right), \omega_{\lambda}^{G}\left(z, T^{m} z, T^{m} z\right), \omega_{\lambda}^{G}\left(z, T^{m} x, T^{m} y\right), \\
\omega_{\lambda}^{G}\left(z, T^{m} x, T^{m} z\right), \omega_{\lambda}^{G}\left(T^{m} x, T^{m} x, T^{m} y\right), \\
\omega_{\lambda}^{G}\left(T^{m} x, T^{m} x, T^{m} z\right), \frac{\omega_{\lambda}^{G}\left(x, z, T^{m} y\right)\left[1+\omega_{\lambda}^{G}\left(x, z, T^{m} y\right)\right]}{1+\omega_{\lambda}^{G}\left(T^{m} x, y, z\right)+\omega_{\lambda}^{G}\left(x, z, T^{m} y\right)} \\
\frac{\omega_{\lambda}^{G}\left(T^{m} x, T^{m} y, T^{m} z\right)\left[1+\omega_{\lambda}^{G}\left(T^{m} x, y, z\right)+\omega_{\lambda}^{G}\left(T^{m} x, y, T^{m} z\right)\right]}{1+\omega_{\lambda}^{G}\left(T^{m} x, y, T^{m} z\right)+\omega_{\lambda}^{G}\left(T^{m} x, T^{m} y, T^{m} z\right)}
\end{array}\right\}
$$

for all $x, y, z \in A \subseteq X_{\omega}, m=1,2, \ldots, k$. Then $T$ has a unique fixed point for some positive integer $m \geq 1$. This is a variant form of Theorem 3.6 in [14].

Proposition 3.8 Let $\left(X, \omega^{G}\right)$ be a G-complete non-symmetric modular G-metric space satisfying a $\Delta_{3}$-type condition, such that $C_{2} C_{4} \in\left(0, \frac{1}{4 \rho}\right)$ and let $T: \mathfrak{A} \subseteq X_{\omega^{G}} \rightarrow X_{\omega^{G}}$ be a mapping. Suppose that there exists some integer $m \geq 1, \lambda>0$, and $k \in(0,1)$ such that

$$
\omega_{\lambda}^{G}\left(T^{m} x, T^{m} y, T^{m} z\right) \leq k B_{\lambda}(x, y, z) \quad \text { for all } x, y, z \in X_{\omega^{G}}, \lambda>0,
$$

where

$$
\begin{aligned}
& B_{\lambda}(x, y, z) \\
& \quad=k\left\{\begin{array}{l}
\omega_{\lambda}^{G}(x, y, z)+\omega_{\lambda}^{G}\left(x, T^{m} x, T^{m} x\right)+\omega_{\lambda}^{G}\left(y, T^{m} y, T^{m} y\right)+\omega_{\lambda}^{G}\left(z, T^{m} z, T^{m} z\right) \\
+\omega_{\lambda}^{G}\left(T^{m} x, y, z\right)+\omega_{\lambda}^{G}\left(y, T^{m} x, T^{m} y\right)+\omega_{\lambda}^{G}\left(y, T^{m} z, T^{m} z\right)+\omega_{\lambda}^{G}\left(z, T^{m} x, T^{m} x\right) \\
+\omega_{\lambda}^{G}\left(z, T^{m} x, T^{m} z\right)+\omega_{\lambda}^{G}\left(T^{m} x, T^{m} x, T^{m} y\right)+\omega_{\lambda}^{G}\left(T^{m} x, T^{m} x, T^{m} z\right)
\end{array}\right\}
\end{aligned}
$$


for all $x, y, z \in \mathfrak{A} \subseteq X_{\omega^{G}}$, Then $T$ has a unique fixed point for some positive integer $m \geq 1$. This is a variant form of Theorem 3.6 in [14].

Theorem 3.9 Let $\left(X, \omega^{G}\right)$ be a G-complete non-symmetric modular G-metric space satisfying a $\Delta_{3}$-type condition, such that $C_{2} C_{4} \in\left(0, \frac{1}{4 \rho}\right)$ and let $T: X_{\omega^{G}} \rightarrow X_{\omega^{G}}$ be a mapping. Suppose that there exists $\lambda>0$ and $\rho \in(0,1)$ such that

$$
\omega_{\lambda}^{G}(T x, T y, T z) \leq \rho \max \left\{\begin{array}{l}
\omega_{\lambda}^{G}(x, y, z), \omega_{\lambda}^{G}(x, z, T y), \omega_{\lambda}^{G}(T x, y, z), \\
\omega_{\lambda}^{G}(x, T x, y), \omega_{\lambda}^{G}(y, z, T y), \omega_{\lambda}^{G}(x, T x, T x), \\
\omega_{\lambda}^{G}(x, T y, T y), \omega_{\lambda}^{G}(x, T x, z), \omega_{\lambda}^{G}(y, T y, T y), \\
\omega_{\lambda}^{G}(y, T x, T y), \omega_{\lambda}^{G}\left(y, T^{2} x, T y\right), \omega_{\lambda}^{G}(y, T z, T z), \\
\omega_{\lambda}^{G}(z, T x, T x), \omega_{\lambda}^{G}(z, T z, T z), \omega_{\lambda}^{G}(z, T x, T y), \\
\omega_{\lambda}^{G}\left(z, T^{2} x, T z\right), \omega_{\lambda}^{G}\left(T x, T^{2} x, T y\right), \omega_{\lambda}^{G}\left(T x, T^{2} x, T z\right)
\end{array}\right\} .
$$

Then $T$ has a unique fixed point in $X_{\omega^{G}}$ and is modular G-continuous at its fixed point (say u).

Proof Following Theorem 3.1, $T u=u$ and hence $T$ has a unique fixed point in $X_{\omega^{G}}$. Also $T x_{n}=u=T u$ as $n \rightarrow \infty$.

Corollary 3.10 Let $\left(X, \omega^{G}\right)$ be a G-complete non-symmetric modular G-metric space satisfying a $\Delta_{3}$-type condition, such that $C_{2} C_{4} \in\left(0, \frac{1}{4 \rho}\right)$ and let $T: X_{\omega^{G}} \rightarrow X_{\omega^{G}}$ be a mapping. Suppose that there exists some positive integer, $m \geq 1, \lambda>0$, and $\rho \in(0,1)$ such that

$$
\begin{aligned}
& \omega_{\lambda}^{G}\left(T^{m} x, T^{m} y, T^{m} z\right) \\
& \quad \leq \rho \max \left\{\begin{array}{l}
\omega_{\lambda}^{G}(x, y, z), \omega_{\lambda}^{G}\left(x, z, T^{m} y\right), \omega_{\lambda}^{G}\left(T^{m} x, y, z\right), \\
\omega_{\lambda}^{G}\left(x, T^{m} x, y\right), \omega_{\lambda}^{G}\left(y, z, T^{m} y\right), \omega_{\lambda}^{G}\left(x, T^{m} x, T^{m} x\right), \\
\omega_{\lambda}^{G}\left(x, T^{m} y, T^{m} y\right), \omega_{\lambda}^{G}\left(x, T^{m} x, z\right), \omega_{\lambda}^{G}\left(y, T^{m} y, T^{m} y\right), \\
\omega_{\lambda}^{G}\left(y, T^{m} x, T^{m} y\right), \omega_{\lambda}^{G}\left(y, T^{m} x, T^{m} y\right), \omega_{\lambda}^{G}\left(y, T^{m} z, T^{m} z\right), \\
\omega_{\lambda}^{G}\left(z, T^{m} x, T^{m} x\right), \omega_{\lambda}^{G}\left(z, T^{m} z, T^{m} z\right), \omega_{\lambda}^{G}\left(z, T^{m} x, T^{m} y\right), \\
\omega_{\lambda}^{G}\left(z, T^{m} x, T^{m} z\right), \omega_{\lambda}^{G}\left(T^{m} x, T^{m} x, T^{m} y\right), \omega_{\lambda}^{G}\left(T^{m} x, T^{m} x, T^{m} z\right)
\end{array}\right\} .
\end{aligned}
$$

Then $T$ has a unique fixed point in $X_{\omega^{G}}$ and $T^{m}$ is modular G-continuous at its fixed point (say $u$ ) for some positive integer $m \geq 1$.

Proof $T^{m} u$ has a unique fixed point $u$ say, that is $T^{m} u=u$. Now we see that $T u=T\left(T^{m} u\right)=$ $T^{m+1} u=T^{m}(T u)$. But $T u$ is another fixed point for $T^{m} u$ and by uniqueness of the limit, $T u=u$. By Corollary 3.6, $T^{m} u$ is modular G-continuous at $u$, i.e. $T^{m} x_{n}=u=T^{m} u$ for some positive integer $m \geq 1$.

Corollary 3.11 Let $\left(X, \omega^{G}\right)$ be a G-complete non-symmetric modular G-metric space satisfying a $\Delta_{3}$-type condition, such that $C_{2} C_{4} \in\left(0, \frac{1}{4 \rho}\right)$ and let $T: X_{\omega^{G}} \rightarrow X_{\omega^{G}}$ be a mapping. 
Suppose that there exists $\lambda>0$ and $\rho \in(0,1)$ such that

$$
\omega_{\lambda}^{G}(T x, T y, T z) \leq \rho K_{\lambda}(x, y, z) \quad \text { for all } x, y, z \in X_{\omega^{G}}, \lambda>0
$$

where

$$
\left.\begin{array}{l}
K_{\lambda}(x, y, z) \\
=\max \left\{\begin{array}{l}
\omega_{\lambda}^{G}(x, y, z), \frac{\omega_{\lambda}^{G}(y, z, T y)\left[1+\omega_{\lambda}^{G}(x, T x, y)\right]}{1+\omega_{\lambda}^{G}(x, y, z)}, \frac{\omega_{\lambda}^{G}(x, T y, z)\left[1+\omega_{\lambda}^{G}(x, T x, y)\right]}{1+\omega_{\lambda}^{G}(x, y, z)}, \\
\frac{\omega_{\lambda}^{G}(x, T y, z)\left[1+\omega_{\lambda}^{G}(x, y, z)\right]}{1+\omega_{\lambda}^{G}(x, y, z)+\omega_{\lambda}^{G}(y, T x, z)}, \frac{\omega_{\lambda}^{G}(x, T y, z)\left[1+\omega_{\lambda}^{G}(x, y, T y)\right]}{1+\omega_{\lambda}^{G}(T x, y, z)+\omega_{\lambda}^{G}(x, z, T y)}, \\
\frac{\omega_{\lambda}^{G}(x, z, T y)\left[1+\omega_{\lambda}^{G}(x, z, T y)\right]}{1+\omega_{\lambda}^{G}(T x, y, z)+\omega_{\lambda}^{G}(x, z, T y)}, \\
1+T_{\lambda}{ }^{G}(T x, T y, T z)\left[1+\omega_{\lambda}^{G}(T x, y, z)+\omega_{\lambda}^{G}(T x, y, T z)\right]
\end{array}\right.
\end{array}\right\} .
$$

Then $T$ has a unique fixed point in $X_{\omega^{G}}$ and is modular G-continuous at its fixed point (say $u)$.

Proof Following Theorem 3.1, we have $\omega_{\lambda}^{G}\left(x_{n+1}, x_{n+2}, x_{n+2}\right)=\omega_{\lambda}^{G}(T x, T y, T z)=\omega_{\lambda}^{G}\left(T x_{n}\right.$, $\left.T x_{n+1}, T x_{n+1}\right)$, so that inequality (3.73) becomes

$$
\omega_{\lambda}^{G}\left(x_{n+1}, x_{n+2}, x_{n+2}\right) \leq \rho K_{\lambda}\left(x_{n}, x_{n+1}, x_{n+1}\right) \quad \forall x_{n}, x_{n+1} \in X_{\omega^{G}}, \lambda>0,
$$

where

$$
\left.\begin{array}{l}
K_{\lambda}\left(x_{n}, x_{n+1}, x_{n+1}\right) \\
=\max \left\{\begin{array}{l}
\omega_{\lambda}^{G}\left(x_{n}, x_{n+1}, x_{n+1}\right), \frac{\omega_{\lambda}^{G}\left(x_{n+1}, x_{n+1}, T x_{n+1}\right)\left[1+\omega_{\lambda}^{G}\left(x_{n}, T x_{n}, x_{n+1}\right)\right]}{1+\omega_{\lambda}^{G}\left(x_{n}, x_{n+1}, x_{n+1}\right)}, \\
\frac{\omega_{\lambda}^{G}\left(x_{n}, T x_{n+1}, x_{n+1}\right)\left[1+\omega_{\lambda}^{G}\left(x_{n}, T x_{n}, x_{n+1}\right)\right]}{1+\omega_{\lambda}^{G}\left(x_{n}, x_{n+1}, x_{n+1}\right)}, \\
\frac{\omega_{\lambda}^{G}\left(x_{n}, T x_{n+1}, x_{n+1}\right)\left[1+\omega_{\lambda}^{G}\left(x_{n}, x_{n+1}, x_{n+1}\right)\right]}{1+\omega_{\lambda}^{G}\left(x_{n}, x_{n+1}, x_{n+1}\right)+\omega_{\lambda}^{G}\left(x_{n+1}, T x_{n}, x_{n+1}\right)}, \\
\frac{\omega_{\lambda}^{G}\left(x_{n}, x_{n+1}, T x_{n+1}\right)\left[1+\omega_{\lambda}^{G}\left(x_{n}, x_{n+1}, T x_{n+1}\right)\right]}{1+\omega_{\lambda}^{G}\left(T x_{n}, x_{n+1}, x_{n+1}\right)+\omega_{\lambda}^{G}\left(x_{n}, x_{n+1}, T x_{n+1}\right)}, \\
\frac{\omega_{\lambda}^{G}\left(T^{2} x_{n}, T x_{n+1}, T x_{n+1}\right)\left[1+\omega_{\lambda}^{G}\left(T x_{n}, x_{n+1}, x_{n+1}\right)+\omega_{\lambda}^{G}\left(T x_{n}, x_{n+1}, T x_{n+1}\right)\right]}{1+\omega_{\lambda}^{G}\left(T x_{n}, x_{n+1}, T x_{n+1}\right)+\omega_{\lambda}^{G}\left(T x_{n}, T x_{n+1}, T x_{n+1}\right)}
\end{array}\right.
\end{array}\right\}
$$




$$
\begin{aligned}
& =\max \left\{\begin{array}{l}
\omega_{\lambda}^{G}\left(x_{n}, x_{n+1}, x_{n+1}\right), \frac{\omega_{\lambda}^{G}\left(x_{n+1}, x_{n+1}, x_{n+2}\right)\left[1+\omega_{\lambda}^{G}\left(x_{n}, x_{n+1}, x_{n+1}\right)\right]}{1+\omega_{\lambda}^{G}\left(x_{n}, x_{n+1}, x_{n+1}\right)}, \\
\frac{\omega_{\lambda}^{G}\left(x_{n}, x_{n+2}, x_{n+1}\right)\left[1+\omega_{\lambda}^{G}\left(x_{n}, x_{n+1}, x_{n+1}\right)\right]}{1+\omega_{\lambda}^{G}\left(x_{n}, x_{n+1}, x_{n+1}\right)}, \\
\frac{\omega_{\lambda}^{G}\left(x_{n}, x_{n+2}, x_{n+1}\right)\left[1+\omega_{\lambda}^{G}\left(x_{n}, x_{n+1}, x_{n+1}\right)\right]}{1+\omega_{\lambda}^{G}\left(x_{n}, x_{n+1}, x_{n+1}\right)+\omega_{\lambda}^{G}\left(x_{n+1}, x_{n+1}, x_{n+1}\right)}, \\
\frac{\omega_{\lambda}^{G}\left(x_{n}, x_{n+2}, x_{n+1}\right)\left[1+\omega_{\lambda}^{G}\left(x_{n}, x_{n+1}, x_{n+2}\right)\right]}{1+\omega_{\lambda+1}^{G}\left(x_{n+1}, x_{n+1}, x_{n+1}\right)+\omega_{\lambda}^{G}\left(x_{n}, x_{n+1}, x_{n+2}\right)}, \\
\frac{\omega_{\lambda}^{G}\left(x_{n+2}, x_{n+2}, x_{n+2}\right)\left[1+\omega_{\lambda}^{G}\left(x_{n}, x_{n+1}, x_{n+2}\right)\right]}{1+\omega_{\lambda}^{G}\left(x_{n+1}, x_{n+1}, x_{n+2}\right)+\omega_{\lambda}^{G}\left(x_{n+2}, x_{n+2}, x_{n+2}\right)},
\end{array}\right\} \\
& =\max \left\{\begin{array}{l}
\omega_{\lambda}^{G}\left(x_{n}, x_{n+1}, x_{n+1}\right), \omega_{\lambda}^{G}\left(x_{n+1}, x_{n+1}, x_{n+2}\right), \\
\omega_{\lambda}^{G}\left(x_{n}, x_{n+2}, x_{n+1}\right), \omega_{\lambda}^{G}\left(x_{n}, x_{n+2}, x_{n+1}\right), \\
\omega_{\lambda}^{G}\left(x_{n}, x_{n+2}, x_{n+1}\right), \omega_{\lambda}^{G}\left(x_{n}, x_{n+1}, x_{n+2}\right), \\
\omega_{\lambda}^{G}\left(x_{n+2}, x_{n+2}, x_{n+2}\right)
\end{array}\right\} \\
& =\max \left\{\begin{array}{l}
\omega_{\lambda}^{G}\left(x_{n}, x_{n+1}, x_{n+1}\right), \omega_{\lambda}^{G}\left(x_{n+1}, x_{n+1}, x_{n+2}\right), \\
\omega_{\lambda}^{G}\left(x_{n}, x_{n+2}, x_{n+1}\right), \omega_{\lambda}^{G}\left(x_{n}, x_{n+1}, x_{n+2}\right), \\
\omega_{\lambda}^{G}\left(x_{n+2}, x_{n+2}, x_{n+2}\right)
\end{array}\right\} .
\end{aligned}
$$

By Theorem 3.1, $T$ has a unique fixed point in $X_{\omega^{G}}$ and is modular G-continuous at its fixed point $u$.

Corollary 3.12 Let $\left(X, \omega^{G}\right)$ be a G-complete non-symmetric modular G-metric space satisfying a $\Delta_{3}$-type condition, such that $C_{2} C_{4} \in\left(0, \frac{1}{4 \rho}\right)$ and let $T: X_{\omega^{G}} \rightarrow X_{\omega^{G}}$ be a mapping. Suppose that there exists some positive integer, $m \geq 1, \lambda>0$, and $\rho \in(0,1)$ such that

$$
\omega_{\lambda}^{G}\left(T^{m} x, T^{m} y, T^{m} z\right) \leq \rho W_{\lambda}(x, y, z) \quad \text { for all } x, y, z \in X_{\omega^{G}}, \lambda>0,
$$

where

$$
\begin{aligned}
& W_{\lambda}(x, y, z) \\
& =\max \left\{\begin{array}{l}
\omega_{\lambda}^{G}(x, y, z), \frac{\omega_{\lambda}^{G}\left(y, z, T^{m} y\right)\left[1+\omega_{\lambda}^{G}\left(x, T^{m} x, y\right)\right]}{1+\omega_{\lambda}^{G}(x, y, z)}, \\
\frac{\omega_{\lambda}^{G}\left(x, T^{m} y, z\right)\left[1+\omega_{\lambda}^{G}\left(x, T^{m} x, y\right)\right]}{1+\omega_{\lambda}^{G}(x, y, z)}, \\
\frac{\omega_{\lambda}^{G}\left(x, T^{m} y, z\right)\left[1+\omega_{\lambda}^{G}(x, y, z)\right]}{1+\omega_{\lambda}^{G}(x, y, z)+\omega_{\lambda}^{G}\left(y, T^{m} x, z\right)} \frac{\omega_{\lambda}^{G}\left(x, T^{m} y, z\right)\left[1+\omega_{\lambda}^{G}\left(x, y, T^{m} y\right)\right]}{\left.1+T^{m} x, y, z\right)+\omega_{\lambda}^{G}\left(x, z, T^{m} y\right)}, \\
\frac{\omega_{\lambda}^{G}\left(T^{m} x, T^{m} y, T^{m} z\right)\left[1+\omega_{\lambda}^{G}\left(x, z, T^{m} y\right)\right]}{1+\omega_{\lambda}^{G}\left(T^{m} x, y, T^{m} z\right)+\omega_{\lambda}^{G}\left(T^{m} x, T^{m} y, T^{m} z\right)},
\end{array}\right\} .
\end{aligned}
$$


Then $T$ has a unique fixed point in $X_{\omega^{G}}$ and $T^{m}$ is modular G-continuous at its fixed point u some positive integer, $m \geq 1$.

Proof By Corollary 3.11, $T$ has a unique fixed point in $X_{\omega^{G}}$ and $T^{m}$ is modular Gcontinuous its fixed point $u$ some positive integer, $m \geq 1$.

If we add some terms to Theorem 3.9 using condition (4) of Definition 2.1, we have the following result.

Theorem 3.13 Let $\left(X, \omega^{G}\right)$ be a G-complete G-modular metric space satisfying a $\Delta_{3}$-type condition, such that $C_{2} \in\left(0, \frac{1}{4 \rho}\right), \lambda>0$, and let $T: X_{\omega^{G}} \rightarrow X_{\omega^{G}}$ be a mapping for which the following condition holds:

$$
\begin{aligned}
& \omega_{\lambda}^{G}(T x, T y, T z) \\
& \quad \leq \rho \max \left\{\begin{array}{l}
\omega_{\lambda}^{G}(x, y, z), \omega_{\lambda}^{G}(x, T x, y), \omega_{\lambda}^{G}(x, T x, T x), \omega_{\lambda}^{G}(x, T x, z), \\
\omega_{\lambda}^{G}(y, T y, T y), \omega_{\lambda}^{G}(y, T z, T z), \omega_{\lambda}^{G}\left(y, T^{2} x, T y\right), \omega_{\lambda}^{G}(z, T x, T x), \\
\omega_{\lambda}^{G}(z, T z, T z), \omega_{\lambda}^{G}\left(z, T^{2} x, T z\right), \omega_{\lambda}^{G}\left(T x, T^{2} x, T y\right), \omega_{\lambda}^{G}\left(T x, T^{2} x, T z\right)
\end{array}\right\},
\end{aligned}
$$

where $\rho \in(0,1)$. Then $T$ has a unique fixed point in $X_{\omega^{G}}$ and $T$ is modular G-continuous at (say $u)$.

Proof Let $x_{0} \in X_{\omega^{G}}$ be arbitrary. We generate the sequence of iteration of $T$ based on $x_{0} \in X_{\omega^{G}}$ as follows:

$$
\begin{gathered}
T x_{0}=x_{1} \\
T x_{1}=x_{2} \\
\vdots \\
T x_{n}=x_{n+1}
\end{gathered}
$$

for all $n \in \mathbb{N}$. If there exists some $n_{0} \in \mathbb{N}$ such that $x_{n_{0}+1}=x_{n_{0}}$ then $x_{0}$ is a fixed point of $T$. Now for all $n \in \mathbb{N}, x_{n+1} \neq x_{n}$ and $\lambda>0$, take $x=x_{n}$ and $y=x_{n+1}=z$, then we have $\omega_{\lambda}^{G}\left(x_{n+1}, x_{n+2}, x_{n+2}\right)=\omega_{\lambda}^{G}(T x, T y, T z)=\omega_{\lambda}^{G}\left(T x_{n}, T x_{n+1}, T x_{n+1}\right)$ so that

$$
\begin{aligned}
& \omega_{\lambda}^{G}\left(x_{n+1}, x_{n+2}, x_{n+2}\right) \\
& =\omega_{\lambda}^{G}\left(T x_{n}, T x_{n+1}, T x_{n+1}\right) \\
& \quad \leq \rho \max \left\{\begin{array}{l}
\omega_{\lambda}^{G}\left(x_{n}, x_{n+1}, x_{n+1}\right), \omega_{\lambda}^{G}\left(x_{n}, T x_{n}, x_{n+1}\right), \omega_{\lambda}^{G}\left(x_{n}, T x_{n}, T x_{n}\right), \\
\omega_{\lambda}^{G}\left(x_{n}, T x_{n}, x_{n+1}\right), \omega_{\lambda}^{G}\left(x_{n+1}, T x_{n+1}, T x_{n+1}\right), \omega_{\lambda}^{G}\left(x_{n+1}, T x_{n+1}, T x_{n+1}\right), \\
\omega_{\lambda}^{G}\left(x_{n+1}, T^{2} x_{n}, T x_{n+1}\right), \omega_{\lambda}^{G}\left(x_{n+1}, T x_{n}, T x_{n}\right), \omega_{\lambda}^{G}\left(x_{n+1}, T x_{n+1}, T x_{n+1}\right), \\
\omega_{\lambda}^{G}\left(x_{n+1}, T^{2} x_{n}, T x_{n+1}\right), \omega_{\lambda}^{G}\left(T x_{n}, T^{2} x_{n}, T x_{n+1}\right), \omega_{\lambda}^{G}\left(T x_{n}, T^{2} x_{n}, T x_{n+1}\right)
\end{array}\right\}
\end{aligned}
$$




$$
\begin{aligned}
& =\rho \max \left\{\begin{array}{l}
\omega_{\lambda}^{G}\left(x_{n}, x_{n+1}, x_{n+1}\right), \omega_{\lambda}^{G}\left(x_{n}, x_{n+1}, x_{n+1}\right), \omega_{\lambda}^{G}\left(x_{n}, x_{n+1}, x_{n+1}\right), \\
\omega_{\lambda}^{G}\left(x_{n}, x_{n+1}, x_{n+1}\right), \omega_{\lambda}^{G}\left(x_{n+1}, x_{n+2}, x_{n+2}\right), \omega_{\lambda}^{G}\left(x_{n+1}, x_{n+2}, x_{n+2}\right), \\
\omega_{\lambda}^{G}\left(x_{n+1}, x_{n+2}, x_{n+2}\right), \omega_{\lambda}^{G}\left(x_{n+1}, x_{n+1}, x_{n+1}\right), \omega_{\lambda}^{G}\left(x_{n+1}, x_{n+2}, x_{n+2}\right), \\
\omega_{\lambda}^{G}\left(x_{n+1}, x_{n+2}, x_{n+2}\right), \omega_{\lambda}^{G}\left(x_{n+1}, x_{n+2}, x_{n+2}\right), \omega_{\lambda}^{G}\left(x_{n+1}, x_{n+2}, x_{n+2}\right)
\end{array}\right\} \\
& =\rho \max \left\{\omega_{\lambda}^{G}\left(x_{n}, x_{n+1}, x_{n+1}\right), \omega_{\lambda}^{G}\left(x_{n+1}, x_{n+2}, x_{n+2}\right)\right\} .
\end{aligned}
$$

We have

$$
\omega_{\lambda}^{G}\left(x_{n+1}, x_{n+2}, x_{n+2}\right) \leq \rho \omega_{\lambda}^{G}\left(x_{n}, x_{n+1}, x_{n+1}\right)
$$

Therefore,

$$
\begin{aligned}
\omega_{\lambda}^{G}\left(x_{n+1}, x_{n+2}, x_{n+2}\right) & \leq \rho \omega_{\lambda}^{G}\left(x_{n}, x_{n+1}, x_{n+1}\right) \\
& \leq \rho^{2} \omega_{\lambda}^{G}\left(x_{n-1}, x_{n}, x_{n}\right) \\
& \leq \rho^{3} \omega_{\lambda}^{G}\left(x_{n-2}, x_{n-1}, x_{n-1}\right) \\
& \vdots \\
& \leq \rho^{n} \omega_{\lambda}^{G}\left(x_{0}, x_{1}, x_{1}\right) .
\end{aligned}
$$

But $\sum_{n \in \mathbb{N}} \rho^{n}<+\infty$. Now $\sum_{n \in \mathbb{N}} \omega_{\lambda}^{G}\left(x_{n+1}, x_{n+2}, x_{n+2}\right) \leq \omega_{\lambda}^{G}\left(x_{0}, x_{1}, x_{1}\right) \sum_{n \in \mathbb{N}} \rho^{n}<+\infty$ for all $\lambda>0$. Suppose that $m, n \in \mathbb{N}$ and $m>n \in \mathbb{N}$. Observe that, for any arbitrary $\epsilon$, using the rectangle inequality repeatedly and condition (2) of Proposition 2.2, we have

$$
\begin{aligned}
\omega_{\lambda}^{G}\left(x_{n}, x_{m}, x_{m}\right) \leq & \omega_{\frac{\lambda}{m-n}}^{G}\left(x_{n}, x_{n+1}, x_{n+1}\right)+\omega_{\frac{\lambda}{m-n}}^{G}\left(x_{n+1}, x_{n+2}, x_{n+2}\right)+\omega_{\frac{\lambda}{m-n}}^{G}\left(x_{n+2}, x_{n+3}, x_{n+3}\right) \\
& +\omega_{\frac{\lambda}{m-n}}^{G}\left(x_{n+3}, x_{n+4}, x_{n+4}\right)+\cdots+\omega_{\frac{\lambda}{m-n}}^{G}\left(x_{m-1}, x_{m}, x_{m}\right) \\
\leq & \omega_{\frac{\lambda}{m}}^{G}\left(x_{n}, x_{n+1}, x_{n+1}\right)+\omega_{\frac{\lambda}{m}}^{G}\left(x_{n+1}, x_{n+2}, x_{n+2}\right)+\omega_{\frac{\lambda}{m}}^{G}\left(x_{n+2}, x_{n+3}, x_{n+3}\right) \\
& +\omega_{\frac{\lambda}{m}}^{G}\left(x_{n+3}, x_{n+4}, x_{n+4}\right)+\cdots+\omega_{\frac{\lambda}{m}}^{G}\left(x_{m-1}, x_{m}, x_{m}\right) \\
\leq & \sum_{n=N} \omega_{\lambda}^{G}\left(x_{n+1}, x_{n+2}, x_{n+2}\right) \\
< & \epsilon
\end{aligned}
$$

for all $m>n \geq N$ for some $N \in \mathbb{N}$. As $\epsilon$ is arbitrary, we have

$$
\omega_{\lambda}^{G}\left(x_{n}, x_{m}, x_{m}\right)=0 \quad \text { as } n, m \rightarrow \infty \quad \text { or } \quad \lim _{n, m \rightarrow \infty} \omega_{\lambda}^{G}\left(x_{n}, x_{m}, x_{m}\right)=0
$$

For $n, m, k \in \mathbb{N}$, condition (2) of Proposition 2.2 implies that

$$
\omega_{\lambda}^{G}\left(x_{n}, x_{m}, x_{k}\right) \leq \omega_{\frac{\lambda}{2}}^{G}\left(x_{n}, x_{m}, x_{m}\right)+\omega_{\frac{\lambda}{2}}^{G}\left(x_{k}, x_{m}, x_{m}\right)
$$


so that on taking the limit of both sides of inequality (3.85) as $n, m, l \rightarrow \infty$ and by applying Definition 2.11 and Eq. (3.84), we get

$$
\begin{aligned}
\lim _{n, m, k \rightarrow \infty} \omega_{\lambda}^{G}\left(x_{n}, x_{m}, x_{k}\right) & \leq \lim _{n, m \rightarrow \infty} \omega_{\frac{\lambda}{2}}^{G}\left(x_{n}, x_{m}, x_{m}\right)+\lim _{k, m \rightarrow \infty} \omega_{\lambda}^{G}\left(x_{k}, x_{m}, x_{m}\right) \\
& \leq C_{2} \lim _{n, m \rightarrow \infty} \omega_{\lambda}^{G}\left(x_{n}, x_{m}, x_{m}\right)+C_{2} \lim _{k, m \rightarrow \infty} \omega_{\lambda}^{G}\left(x_{k}, x_{m}, x_{m}\right) \\
& =C_{2}\left(\lim _{n, m \rightarrow \infty} \omega_{\lambda}^{G}\left(x_{n}, x_{m}, x_{m}\right)+\lim _{k, m \rightarrow \infty} \omega_{\lambda}^{G}\left(x_{k}, x_{m}, x_{m}\right)\right) ;
\end{aligned}
$$

thus, we have

$$
\lim _{n, m, k \rightarrow \infty} \omega_{\lambda}^{G}\left(x_{n}, x_{m}, x_{k}\right)=0 \quad \forall \lambda>0
$$

By Equation (3.87), $\left\{x_{n}\right\}_{n \in \mathbb{N}}$ is a modular G-Cauchy sequence.

The completeness of $\left(X, \omega^{G}\right)$ implies that, for any $\lambda>0, \lim _{n, m \rightarrow \infty} \omega_{\lambda}^{G}\left(x_{n}, x_{m}, u\right)=0$, i.e. for any $\epsilon>0$, there exists $n_{0} \in \mathbb{N}$ such that $\omega_{\lambda}^{G}\left(x_{n}, x_{m}, u\right)<\epsilon$ for all $n, m \in \mathbb{N}$ and $n, m \geq$ $n_{0}$, which implies that $\lim _{n \rightarrow \infty} x_{n} \rightarrow u$. Suppose, if possible, that $T u \neq u$, i.e. for $\lambda>0$, $\omega_{\lambda}^{G}(u, T u, T u)>0$ then from inequality (3.76), with $x=x_{n}, y=u=z$, then we have

$$
\omega_{\lambda}^{G}\left(x_{n+1}, T u, T u\right)=\omega_{\lambda}^{G}\left(T x_{n}, T u, T u\right) \leq \rho K_{\lambda}\left(x_{n}, u, u\right) \quad \text { for all } x_{n}, u, \in X_{\omega^{G}}, \lambda>0,
$$

so that

$$
\begin{aligned}
& K_{\lambda}\left(x_{n}, u, u\right) \\
& =\rho \max \left\{\begin{array}{l}
\omega_{\lambda}^{G}\left(x_{n}, u, u\right), \omega_{\lambda}^{G}\left(x_{n}, T x_{n}, u\right), \omega_{\lambda}^{G}\left(x_{n}, T x_{n}, T x_{n}\right), \omega_{\lambda}^{G}\left(x_{n}, T x_{n}, u\right), \\
\omega_{\lambda}^{G}(u, T u, T u), \omega_{\lambda}^{G}(u, T u, T u), \omega_{\lambda}^{G}\left(u, T^{2} x_{n}, T u\right), \omega_{\lambda}^{G}\left(u, T x_{n}, T x_{n}\right),
\end{array}\right\} \\
& =\rho \max \left\{\begin{array}{l}
\omega_{\lambda}^{G}\left(x_{n}, u, u\right), \omega_{\lambda}^{G}\left(x_{n}, x_{n+1}, u\right), \omega_{\lambda}^{G}\left(x_{n}, x_{n+1}, x_{n+1}\right), \omega_{\lambda}^{G}\left(x_{n}, x_{n+1}, u\right), \\
\left.\omega_{\lambda}^{G}(u, T u, T u), \omega_{\lambda}^{G}(u, T u, T u), \omega_{\lambda}^{G}\left(u, x_{n+2}, T u\right), \omega_{\lambda}^{G}\left(u, x_{n+1}, x_{n+1}\right), T u\right), \omega_{\lambda}^{G}\left(T x_{n}, T^{2} x_{n}, T u\right) \\
\omega_{\lambda}^{G}(u, T u, T u), \omega_{\lambda}^{G}\left(u, x_{n+2}, T u\right), \omega_{\lambda}^{G}\left(x_{n+1}, x_{n+2}, T u\right), \omega_{\lambda}^{G}\left(x_{n+1}, x_{n+2}, T u\right)
\end{array}\right\}
\end{aligned}
$$

as $n \rightarrow \infty$, we get

$$
\omega_{\lambda}^{G}(u, T u, T u) \leq \rho \max \left\{\omega_{\lambda}^{G}(u, T u, T u), \omega_{\lambda}^{G}(u, u, T u)\right\} .
$$

Now we consider the following cases.

Case 1. If $\max \left\{\omega_{\lambda}^{G}(u, T u, T u), \omega_{\lambda}^{G}(u, u, T u)\right\}=\omega_{\lambda}^{G}(u, T u, T u)$, then inequality (3.91) becomes

$$
\omega_{\lambda}^{G}(u, T u, T u) \leq \rho \omega_{\lambda}^{G}(u, T u, T u),
$$


which implies that

$$
(1-\rho) \omega_{\lambda}^{G}(u, T u, T u) \leq 0, \quad \rho \in(0,1) .
$$

Hence for all $\lambda>0, T u=u$.

Case 2. If $\max \left\{\omega_{\lambda}^{G}(u, T u, T u), \omega_{\lambda}^{G}(u, u, T u)\right\}=\omega_{\lambda}^{G}(u, u, T u)$, then, by condition (4) of Proposition 2.2, inequality (3.91) becomes

$$
\begin{aligned}
\omega_{\lambda}^{G}(u, T u, T u) & \leq \rho \omega_{\lambda}^{G}(u, u, T u) \\
& \leq 2 \rho \omega_{\frac{\lambda}{2}}^{G}(u, T u, T u) \\
& \leq 2 C_{2} \rho \omega_{\lambda}^{G}(u, T u, T u), \quad C_{2} \in\left(0, \frac{1}{2 \rho}\right),
\end{aligned}
$$

which implies that

$$
\left(1-2 \rho C_{2}\right) \omega_{\lambda}^{G}(u, T u, T u) \leq 0,
$$

for all $\lambda>0$ and $C_{2} \in\left(0, \frac{1}{2 \rho}\right)$, which is a contradiction. Hence, $T u=u$. Following Theorem 3.1 carefully, $T$ has a unique fixed point in $X_{\omega^{G}}$ and $T$ is modular $G$-continuous at say $u$.

Remark 3.7 Let $\left(X, \omega^{G}\right)$ be a $G$-complete $G$-modular metric space satisfying a $\Delta_{3}$-type condition, such that $C_{2} \in\left(0, \frac{1}{2 \rho}\right), \lambda>0$, and let $T: X_{\omega^{G}} \rightarrow X_{\omega^{G}}$ be a mapping for which the following condition holds:

$$
\begin{aligned}
& \omega_{\lambda}^{G}(T x, T y, T z) \\
& \leq \rho \max \left\{\begin{array}{l}
\omega_{\lambda}^{G}(x, y, z), \omega_{\lambda}^{G}(x, T x, y), \omega_{\lambda}^{G}(x, T x, T x), \omega_{\lambda}^{G}(x, T x, z), \\
\omega_{\lambda}^{G}(y, T y, T y), \omega_{\lambda}^{G}(y, T z, T z), \omega_{\lambda}^{G}\left(y, T^{2} x, T y\right), \omega_{\lambda}^{G}(z, T x, T x), \\
\omega_{\lambda}^{G}(z, T z, T z), \omega_{\lambda}^{G}\left(z, T^{2} x, T z\right), \omega_{\lambda}^{G}\left(T x, T^{2} x, T y\right), \omega_{\lambda}^{G}\left(T x, T^{2} x, T z\right)
\end{array}\right\} \\
& =\alpha \omega_{\lambda}^{G}(x, T x, T x)+\beta \omega_{\lambda}^{G}(y, T y, T y)+\gamma \omega_{\lambda}^{G}(z, T z, T z)
\end{aligned}
$$

for $\alpha+\beta+\gamma \in(0,1)$. Then $T$ has a unique fixed point in $X_{\omega^{G}}$ and $T$ is modular Gcontinuous at $u$. In fact this is Theorem 3.2 of [14].

Remark 3.8 Let $\left(X, \omega^{G}\right)$ be a $G$-complete $G$-modular metric space satisfying a $\Delta_{3}$-type condition, such that $C_{2} \in\left(0, \frac{1}{2 \rho}\right), \lambda>0$, and let $T: X_{\omega^{G}} \rightarrow X_{\omega^{G}}$ be a mapping for which the following condition holds:

$$
\begin{aligned}
& \omega_{\lambda}^{G}(T x, T y, T z) \\
& \quad \leq \rho \max \left\{\begin{array}{l}
\omega_{\lambda}^{G}(x, y, z), \omega_{\lambda}^{G}(x, T x, y), \omega_{\lambda}^{G}(x, T x, T x), \omega_{\lambda}^{G}(x, T x, z), \\
\omega_{\lambda}^{G}(y, T y, T y), \omega_{\lambda}^{G}(y, T z, T z), \omega_{\lambda}^{G}\left(y, T^{2} x, T y\right), \omega_{\lambda}^{G}(z, T x, T x), \\
\omega_{\lambda}^{G}(z, T z, T z), \omega_{\lambda}^{G}\left(z, T^{2} x, T z\right), \omega_{\lambda}^{G}\left(T x, T^{2} x, T y\right), \omega_{\lambda}^{G}\left(T x, T^{2} x, T z\right)
\end{array}\right\}
\end{aligned}
$$




$$
=\alpha \omega_{\lambda}^{G}(x, T x, T x)+\beta \omega_{\lambda}^{G}(y, T y, T y)+\gamma \omega_{\lambda}^{G}(z, T z, T z)+\delta \omega_{\lambda}^{G}(x, y, z)
$$

for $\alpha+\beta+\gamma+\delta \in(0,1)$. Then $T$ has a unique fixed point in $X_{\omega^{G}}$ and $T$ is modular $G$ continuous at $u$. This is Theorem 3.3 of [14].

Corollary 3.14 Let $\left(X, \omega^{G}\right)$ be a G-complete modular G-metric space satisfying a $\Delta_{3}$-type condition, such that $C_{2} \in\left(0, \frac{1}{2 \rho}\right), \lambda>0, m=1,2, \ldots, k$, and let $T: X_{\omega^{G}} \rightarrow X_{\omega^{G}}$ be a mapping for which the following condition holds:

$$
\begin{aligned}
& \omega_{\lambda}^{G}\left(T^{m} x, T^{m} y, T^{m} z\right) \\
& \leq \rho \max \left\{\begin{array}{l}
\omega_{\lambda}^{G}(x, y, z), \omega_{\lambda}^{G}\left(x, T^{m} x, y\right), \omega_{\lambda}^{G}\left(x, T^{m} x, T^{m} x\right), \\
\omega_{\lambda}^{G}\left(x, T^{m} x, z\right), \omega_{\lambda}^{G}\left(y, T^{m} y, T^{m} y\right), \omega_{\lambda}^{G}\left(y, T^{m} z, T^{m} z\right), \\
\omega_{\lambda}^{G}\left(y, T^{m} x, T^{m} y\right), \omega_{\lambda}^{G}\left(z, T^{m} x, T^{m} x\right), \omega_{\lambda}^{G}\left(z, T^{m} z, T^{m} z\right), \\
\omega_{\lambda}^{G}\left(z, T^{m} x, T^{m} z\right), \omega_{\lambda}^{G}\left(T^{m} x, T^{m} x, T^{m} y\right), \omega_{\lambda}^{G}\left(T^{m} x, T^{m} x, T^{m} z\right)
\end{array}\right\},
\end{aligned}
$$

where $\rho \in(0,1)$. Then $T$ has a unique fixed point in $X_{\omega^{G}}$ and $T$ is modular G-continuous at its fixed point (say $u)$.

Proof $T^{m} u$ has a unique fixed point $u$ say, that is $T^{m} u=u$. Now we see that $T u=T\left(T^{m} u\right)=$ $T^{m+1} u=T^{m}(T u)$. But $T u$ is another fixed point for $T^{m} u$ and by uniqueness of limit, $T u=u$. By Theorem 3.13, $T^{m} u$ is modular G-continuous at $u$, i.e. $T^{m} x_{n}=u=T^{m} u$.

Remark 3.9 Let $\left(X, \omega^{G}\right)$ be a G-complete modular $G$-metric space satisfying a $\Delta_{3}$-type condition, such that $C_{2} \in\left(0, \frac{1}{2 \rho}\right)$ and there is some positive integer, $m \geq 1, \lambda>0$, and let $T: X_{\omega^{G}} \rightarrow X_{\omega^{G}}$ be a mapping such that

$$
\begin{aligned}
& \omega_{\lambda}^{G}\left(T^{m} x, T^{m} y, T^{m} z\right) \\
& \leq \rho \max \left\{\begin{array}{l}
\omega_{\lambda}^{G}(x, y, z), \omega_{\lambda}^{G}\left(x, T^{m} x, y\right), \omega_{\lambda}^{G}\left(x, T^{m} x, T^{m} x\right), \\
\omega_{\lambda}^{G}\left(x, T^{m} x, z\right), \omega_{\lambda}^{G}\left(y, T^{m} y, T^{m} y\right), \omega_{\lambda}^{G}\left(y, T^{m} z, T^{m} z\right), \\
\omega_{\lambda}^{G}\left(y, T^{m} x, T^{m} y\right), \omega_{\lambda}^{G}\left(z, T^{m} x, T^{m} x\right), \omega_{\lambda}^{G}\left(z, T^{m} z, T^{m} z\right), \\
\omega_{\lambda}^{G}\left(z, T^{m} x, T^{m} z\right), \omega_{\lambda}^{G}\left(T^{m} x, T^{m} x, T^{m} y\right), \omega_{\lambda}^{G}\left(T^{m} x, T^{m} x, T^{m} z\right)
\end{array}\right\} \\
& =\alpha \omega_{\lambda}^{G}\left(x, T^{m} x, T^{m} x\right)+\beta \omega_{\lambda}^{G}\left(y, T^{m} y, T^{m} y\right) \\
& +\gamma \omega_{\lambda}^{G}\left(z, T^{m} z, T^{m} z\right)+\delta \omega_{\lambda}^{G}(x, y, z),
\end{aligned}
$$

for $\alpha+\beta+\gamma+\delta \in(0,1)$. Then $T$ has a unique fixed point in $X_{\omega^{G}}$ and $T$ is modular $G$ continuous at its fixed point $u$. This is a variant form of Theorem 3.3 in [14].

\section{Fixed point theorems of weakly compatible mappings in modular G-metric space}

In this section we will briefly investigate fixed point theorems of compatible mappings in modular G-metric space. 
Theorem 4.1 ([15]) Let $\left(X, \omega^{G}\right)$ be a modular G-metric space and $g, h: X \rightarrow X$ be two self-mappings satisfying either

$$
\omega_{\lambda}^{G}(g x, g y, g z) \leq \phi\left(\max \left\{\omega_{\lambda}^{G}(h x, g x, g x), \omega_{\lambda}^{G}(h y, g y, g y), \omega_{\lambda}^{G}(h z, g z, g z)\right\}\right),
$$

or

$$
\omega_{\lambda}^{G}(g x, g y, g z) \leq \phi\left(\max \left\{\omega_{\lambda}^{G}(h x, h x, g x), \omega_{\lambda}^{G}(h y, h y, g y), \omega_{\lambda}^{G}(h z, h z, g z)\right\}\right),
$$

for all $x, y, x \in X_{\omega^{G}}$ and $\lambda>0$. If the range of $h$ contains the range of $g$ and $h\left(X_{\omega^{G}}\right)$ is a complete subspace of $X$, then $g$ and $h$ has a unique point of coincidence in $X_{\omega}$. Furthermore, if $g$, $h$ are weakly compatible, then $g$ and $h$ have a unique common fixed point.

Theorem 4.2 Let $\left(X, \omega^{G}\right)$ be a modular G-metric space satisfying a $\Delta_{3}$-type condition, $C_{2}+C_{4} \in\left(0, \frac{1}{k}\right)$ and $T, S: X_{\omega^{G}} \rightarrow X_{\omega^{G}}$ be two self-mappings for which $T\left(X_{\omega^{G}}\right) \subseteq S\left(X_{\omega^{G}}\right)$, where $S\left(X_{\omega^{G}}\right)$ is a G-complete subspace of $X_{\omega^{G}}$. Suppose that, for all $\lambda>0$, the following condition holds:

$$
\omega_{\lambda}^{G}(T x, T y, T z) \leq k \max \left\{\begin{array}{l}
\omega_{\lambda}^{G}(S x, S y, S z), \omega_{\lambda}^{G}(T x, T x, S z), \\
\omega_{\lambda}^{G}(T y, T y, S y), \omega_{\lambda}^{G}(S y, T z, S z), \\
\omega_{\lambda}^{G}(S y, T x, S y), \omega_{\lambda}^{G}(S z, T z, T z), \\
\omega_{\lambda}^{G}(T z, S x, T z), \omega_{\lambda}^{G}(T x, S x, S y)
\end{array}\right\},
$$

where $k<1$, for all $x, y, z \in X_{\omega^{G}}$. Then $T, S$ has a unique coincidence point in $X_{\omega^{G}}$. Moreover, if $T$ and $S$ are weakly compatible, then $T$ and $S$ have a unique common fixed point in $X_{\omega^{G}}$.

Proof Let $x_{0} \in X_{\omega^{G}}$ be an arbitrary point. Since $T\left(X_{\omega^{G}}\right) \subseteq S\left(X_{\omega^{G}}\right)$, there exists $x_{1} \in X_{\omega^{G}}$ such that $T x_{0}=S x_{1}$. Continuing in this way we have $T x_{n}=S x_{n+1}$, for $n \in \mathbb{N}$. So for any $\lambda>0, \omega_{\lambda}^{G}\left(S x_{n+1}, S x_{n}, S x_{n}\right) \leq \omega_{\lambda}^{G}\left(T x_{n}, T x_{n-1}, T x_{n-1}\right)$. From inequality (4.3), we have

$$
\begin{aligned}
& \omega_{\lambda}^{G}\left(S x_{n+1}, S x_{n}, S x_{n}\right) \\
& \leq k \max \left\{\begin{array}{l}
\omega_{\lambda}^{G}\left(S x_{n}, S x_{n-1}, S x_{n-1}\right), \omega_{\lambda}^{G}\left(T x_{n}, T x_{n}, S x_{n-1}\right), \\
\omega_{\lambda}^{G}\left(T x_{n-1}, T x_{n-1}, S x_{n-1}\right), \omega_{\lambda}^{G}\left(S x_{n-1}, T x_{n-1}, S x_{n-1}\right), \\
\omega_{\lambda}^{G}\left(S x_{n-1}, T x_{n}, S x_{n-1}\right), \omega_{\lambda}^{G}\left(S x_{n-1}, T x_{n-1}, T x_{n-1}\right), \\
\omega_{\lambda}^{G}\left(T x_{n-1}, S x_{n}, T x_{n-1}\right), \omega_{\lambda}^{G}\left(T x_{n}, S x_{n}, S x_{n-1}\right)
\end{array}\right\} \\
& =k \max \left\{\begin{array}{l}
\omega_{\lambda}^{G}\left(S x_{n}, S x_{n-1}, S x_{n-1}\right), \omega_{\lambda}^{G}\left(S x_{n+1}, S x_{n+1}, S x_{n-1}\right), \\
\omega_{\lambda}^{G}\left(S x_{n}, S x_{n}, S x_{n-1}\right), \omega_{\lambda}^{G}\left(S x_{n-1}, S x_{n}, S x_{n-1}\right), \\
\omega_{\lambda}^{G}\left(S x_{n-1}, S x_{n+1}, S x_{n-1}\right), \omega_{\lambda}^{G}\left(S x_{n-1}, S x_{n}, S x_{n}\right), \\
\omega_{\lambda}^{G}\left(S x_{n}, S x_{n}, S x_{n}\right), \omega_{\lambda}^{G}\left(S x_{n+1}, S x_{n}, S x_{n-1}\right)
\end{array}\right\},
\end{aligned}
$$


using condition (4) of Definition 2.2 which we simplify as

$$
\omega_{\lambda}^{G}\left(S x_{n+1}, S x_{n}, S x_{n}\right) \leq k \max \left\{\begin{array}{l}
\omega_{\lambda}^{G}\left(S x_{n}, S x_{n-1}, S x_{n-1}\right), \omega_{\lambda}^{G}\left(S x_{n+1}, S x_{n+1}, S x_{n-1}\right), \\
\omega_{\lambda}^{G}\left(S x_{n}, S x_{n}, S x_{n-1}\right), \omega_{\lambda}^{G}\left(S x_{n+1}, S x_{n-1}, S x_{n-1}\right), \\
\omega_{\lambda}^{G}\left(S x_{n+1}, S x_{n}, S x_{n-1}\right)
\end{array}\right\}
$$

here we have the following cases.

Case 1. If the right hand side of inequality (4.6) is $k \omega_{\lambda}^{G}\left(S x_{n}, S x_{n-1}, S x_{n-1}\right)$, then we have

$$
\omega_{\lambda}^{G}\left(S x_{n+1}, S x_{n}, S x_{n}\right) \leq k \omega_{\lambda}^{G}\left(S x_{n}, S x_{n-1}, S x_{n-1}\right), \quad k \in(0,1)
$$

Case 2. If the right hand side of inequality (4.6) is $k \omega_{\lambda}^{G}\left(S x_{n+1}, S x_{n+1}, S x_{n-1}\right)$, then we have

$$
\omega_{\lambda}^{G}\left(S x_{n+1}, S x_{n}, S x_{n}\right) \leq k \omega_{\lambda}^{G}\left(S x_{n+1}, S x_{n+1}, S x_{n-1}\right)
$$

using conditions (6), (3) of Proposition 2.2 and Definition 2.11, we have

$$
\begin{aligned}
\omega_{\lambda}^{G}\left(S x_{n+1}, S x_{n}, S x_{n}\right) \leq & k \omega_{\lambda}^{G}\left(S x_{n+1}, S x_{n+1}, S x_{n-1}\right) \\
\leq & k\left\{\omega_{\frac{\lambda}{2}}^{G}\left(S x_{n+1}, S x_{n}, S x_{n}\right)+\omega_{\frac{\lambda}{4}}^{G}\left(S x_{n+1}, S x_{n}, S x_{n}\right)\right. \\
& \left.+\omega_{\frac{\lambda}{4}}^{G}\left(S x_{n}, S x_{n}, S x_{n-1}\right)\right\} \\
\leq & k\left\{C_{2} \omega_{\lambda}^{G}\left(S x_{n+1}, S x_{n}, S x_{n}\right)+C_{4} \omega_{\lambda}^{G}\left(S x_{n+1}, S x_{n}, S x_{n}\right)\right. \\
& \left.+C_{4} \omega_{\lambda}^{G}\left(S x_{n}, S x_{n}, S x_{n-1}\right)\right\} \\
\leq & k\left\{\left(C_{2}+C_{4}\right) \omega_{\lambda}^{G}\left(S x_{n+1}, S x_{n}, S x_{n}\right)+2 C_{4} \omega_{\frac{\lambda}{2}}^{G}\left(S x_{n}, S x_{n-1}, S x_{n-1}\right)\right\} \\
\leq & k\left\{\left(C_{2}+C_{4}\right) \omega_{\lambda}^{G}\left(S x_{n+1}, S x_{n}, S x_{n}\right)+2 C_{2} C_{4} \omega_{\lambda}^{G}\left(S x_{n}, S x_{n-1}, S x_{n-1}\right)\right\} .
\end{aligned}
$$

Hence

$$
\omega_{\lambda}^{G}\left(S x_{n+1}, S x_{n}, S x_{n}\right) \leq p \omega_{\lambda}^{G}\left(S x_{n}, S x_{n-1}, S x_{n-1}\right)
$$

where $p=\frac{2 k C_{2} C_{4}}{1-k\left(C_{2}+C_{4}\right)}<1$, and $C_{2}+C_{4} \in\left(0, \frac{1}{k}\right)$.

Case 3. If the right hand side of inequality (4.6) is $k \omega_{\lambda}^{G}\left(S x_{n}, S x_{n}, S x_{n-1}\right)$, then we have

$$
\omega_{\lambda}^{G}\left(S x_{n+1}, S x_{n}, S x_{n}\right) \leq k \omega_{\lambda}^{G}\left(S x_{n}, S x_{n}, S x_{n-1}\right)
$$

using condition (3) of Proposition 2.2 and Definition 2.11, we have

$$
\begin{aligned}
\omega_{\lambda}^{G}\left(S x_{n+1}, S x_{n}, S x_{n}\right) & \leq k \omega_{\lambda}^{G}\left(S x_{n}, S x_{n}, S x_{n-1}\right) \\
& \leq 2 k \omega_{\frac{\lambda}{2}}^{G}\left(S x_{n}, S x_{n-1}, S x_{n-1}\right) \\
& \leq 2 k C_{2} \omega_{\lambda}^{G}\left(S x_{n}, S x_{n-1}, S x_{n-1}\right),
\end{aligned}
$$


hence

$$
\omega_{\lambda}^{G}\left(S x_{n+1}, S x_{n}, S x_{n}\right) \leq d \omega_{\lambda}^{G}\left(S x_{n}, S x_{n-1}, S x_{n-1}\right),
$$

where $d=2 k C_{2}<1$ and $C_{2} \in\left(0, \frac{1}{k}\right)$.

Case 4. If the right hand side of inequality (4.6) is $k \omega_{\lambda}^{G}\left(S x_{n+1}, S x_{n-1}, S x_{n-1}\right)$, then we have

$$
\omega_{\lambda}^{G}\left(S x_{n+1}, S x_{n}, S x_{n}\right) \leq k \omega_{\lambda}^{G}\left(S x_{n+1}, S x_{n-1}, S x_{n-1}\right),
$$

using condition (5) of Definition 2.1 and Definition 2.11, we have

$$
\begin{aligned}
\omega_{\lambda}^{G}\left(S x_{n+1}, S x_{n}, S x_{n}\right) & \leq k \omega_{\lambda}^{G}\left(S x_{n+1}, S x_{n-1}, S x_{n-1}\right) \\
& \leq k\left(\omega_{\frac{\lambda}{2}}^{G}\left(S x_{n+1}, S x_{n}, S x_{n}\right)+\omega_{\frac{\lambda}{2}}^{G}\left(S x_{n}, S x_{n-1}, S x_{n-1}\right)\right) \\
& \leq k C_{2}\left(\omega_{\lambda}^{G}\left(S x_{n+1}, S x_{n}, S x_{n}\right)+\omega_{\lambda}^{G}\left(S x_{n}, S x_{n-1}, S x_{n-1}\right)\right),
\end{aligned}
$$

so that

$$
\omega_{\lambda}^{G}\left(S x_{n+1}, S x_{n}, S x_{n}\right) \leq b \omega_{\lambda}^{G}\left(S x_{n}, S x_{n-1}, S x_{n-1}\right),
$$

where $b=\frac{k C_{2}}{1-k C_{2}}<1$ and $C_{2} \in\left(0, \frac{1}{k}\right)$.

Case 5. If the right hand side of inequality (4.6) is $k \omega_{\lambda}^{G}\left(S x_{n+1}, S x_{n}, S x_{n-1}\right)$, using condition (1) of Definitions 2.1, 2.11 and condition (6), (3) of Proposition 2.2, then we have

$$
\begin{aligned}
\omega_{\lambda}^{G}\left(S x_{n+1}, S x_{n}, S x_{n}\right) \leq & k \omega_{\lambda}^{G}\left(S x_{n+1}, S x_{n}, S x_{n-1}\right) \\
\leq & k\left\{\omega_{\frac{\lambda}{2}}^{G}\left(S x_{n+1}, S x_{n}, S x_{n}\right)+\omega_{\frac{\lambda}{4}}^{G}\left(S x_{n}, S x_{n}, S x_{n}\right)\right. \\
& \left.+\omega_{\frac{\lambda}{4}}^{G}\left(S x_{n}, S x_{n}, S x_{n-1}\right)\right\} \\
= & k\left\{\omega_{\frac{\lambda}{2}}^{G}\left(S x_{n+1}, S x_{n}, S x_{n}\right)+\omega_{\frac{\lambda}{4}}^{G}\left(S x_{n}, S x_{n}, S x_{n-1}\right)\right\} \\
\leq & k\left\{C_{2} \omega_{\lambda}^{G}\left(S x_{n+1}, S x_{n}, S x_{n}\right)+C_{4} \omega_{\lambda}^{G}\left(S x_{n}, S x_{n}, S x_{n-1}\right)\right\} \\
\leq & k\left\{C_{2} \omega_{\lambda}^{G}\left(S x_{n+1}, S x_{n}, S x_{n}\right)+2 C_{4} \omega_{\frac{\lambda}{2}}^{G}\left(S x_{n}, S x_{n-1}, S x_{n-1}\right)\right\} \\
\leq & k\left\{C_{2} \omega_{\lambda}^{G}\left(S x_{n+1}, S x_{n}, S x_{n}\right)+2 C_{2} C_{4} \omega_{\lambda}^{G}\left(S x_{n}, S x_{n-1}, S x_{n-1}\right)\right\},
\end{aligned}
$$

which implies that

$$
\omega_{\lambda}^{G}\left(S x_{n+1}, S x_{n}, S x_{n}\right) \leq \frac{2 k C_{2} C_{4}}{1-k C_{2}} \omega_{\lambda}^{G}\left(S x_{n}, S x_{n-1}, S x_{n-1}\right) .
$$

Therefore,

$$
\omega_{\lambda}^{G}\left(S x_{n+1}, S x_{n}, S x_{n}\right) \leq r \omega_{\lambda}^{G}\left(S x_{n}, S x_{n-1}, S x_{n-1}\right)
$$

where $r=\frac{2 k C_{2} C_{4}}{1-k C_{2}}<1$ and $C_{2} \in\left(0, \frac{1}{k}\right)$. 
So take $\eta=\max \{k, p, d, b, r\}<1$, so we have

$$
\begin{aligned}
\omega_{\lambda}^{G}\left(S x_{n+1}, S x_{n}, S x_{n}\right) \leq & \eta \omega_{\lambda}^{G}\left(S x_{n}, S x_{n-1}, S x_{n-1}\right) \\
& \vdots \\
\leq & \eta^{n} \omega_{\lambda}^{G}\left(S x_{0}, S x_{1}, S x_{1}\right)
\end{aligned}
$$

But $\sum_{n \in \mathbb{N}} \eta^{n}<+\infty$. Now $\sum_{n \in \mathbb{N}} \omega_{\lambda}^{G}\left(S x_{n+1}, S x_{n}, S x_{n}\right) \leq \omega_{\lambda}^{G}\left(S x_{0}, S x_{1}, S x_{1}\right) \sum_{n \in \mathbb{N}} \eta^{n}<+\infty$ for all $\lambda>0$. Suppose that $m, n \in \mathbb{N}$ and $m>n \in \mathbb{N}$. Observe that, for any arbitrary $\epsilon$, using the rectangle inequality repeatedly and condition (2) of Proposition 2.2, we have

$$
\begin{aligned}
\omega_{\lambda}^{G}\left(S x_{n}, S x_{m}, S x_{m}\right) \leq & \omega_{\frac{\lambda}{m-n}}^{G}\left(S x_{n}, S x_{n+1}, S x_{n+1}\right)+\omega_{\frac{\lambda}{m-n}}^{G}\left(S x_{n+1}, S x_{n+2}, S x_{n+2}\right) \\
& +\omega_{\frac{\lambda}{m-n}}^{G}\left(S x_{n+2}, S x_{n+3}, S x_{n+3}\right) \\
& +\omega_{\frac{\lambda}{m-n}}^{G}\left(S x_{n+3}, S x_{n+4}, S x_{n+4}\right)+\cdots+\omega_{\frac{\lambda}{m-n}}^{G}\left(S x_{m-1}, S x_{m}, S x_{m}\right) \\
\leq & \omega_{\frac{\lambda}{m}}^{G}\left(S x_{n}, S x_{n+1}, S x_{n+1}\right)+\omega_{\frac{\lambda}{m}}^{G}\left(S x_{n+1}, S x_{n+2}, S x_{n+2}\right) \\
& +\omega_{\frac{\lambda}{m}}^{G}\left(S x_{n+2}, S x_{n+3}, S x_{n+3}\right) \\
& +\omega_{\frac{\lambda}{m}}^{G}\left(S x_{n+3}, S x_{n+4}, S x_{n+4}\right)+\cdots+\omega_{\frac{\lambda}{m}}^{G}\left(S x_{m-1}, S x_{m}, S x_{m}\right) \\
\leq & \sum_{n=N} \omega_{\lambda}^{G}\left(S x_{n+1}, S x_{n}, S x_{n}\right) \\
< & \epsilon
\end{aligned}
$$

for all $m>n \geq N$ for some $N \in \mathbb{N}$. As $\epsilon$ is arbitrary, we have

$$
\omega_{\lambda}^{G}\left(S x_{n}, S x_{m}, S x_{m}\right)=0 \quad \text { as } n, m \rightarrow \infty \quad \text { or } \quad \lim _{n, m \rightarrow \infty} \omega_{\lambda}^{G}\left(S x_{n}, S x_{m}, S x_{m}\right)=0
$$

For $n, m, k \in \mathbb{N}$, condition (2) of Proposition 2.2 implies that

$$
\omega_{\lambda}^{G}\left(S x_{n}, S x_{m}, S x_{k}\right) \leq \omega_{\frac{\lambda}{2}}^{G}\left(S x_{n}, S x_{m}, S x_{m}\right)+\omega_{\frac{\lambda}{2}}^{G}\left(S x_{k}, S x_{m}, S x_{m}\right),
$$

so that on taking the limit of both sides of inequality (4.18) as $n, m, l \rightarrow \infty$ and applying Definition 2.11 and Eq. (4.17), we get

$$
\begin{aligned}
& \lim _{n, m, k \rightarrow \infty} \omega_{\lambda}^{G}\left(S x_{n}, S x_{m}, S x_{k}\right) \\
& \leq \lim _{n, m \rightarrow \infty} \omega_{\frac{\lambda}{2}}^{G}\left(S x_{n}, S x_{m}, S x_{m}\right)+\lim _{k, m \rightarrow \infty} \omega_{\frac{\lambda}{2}}^{G}\left(S x_{k}, S x_{m}, S x_{m}\right) \\
& \leq C_{2} \lim _{n, m \rightarrow \infty} \omega_{\lambda}^{G}\left(S x_{n}, S x_{m}, S x_{m}\right)+C_{2} \lim _{k, m \rightarrow \infty} \omega_{\lambda}^{G}\left(S x_{k}, S x_{m}, S x_{m}\right) \\
& \quad=C_{2}\left(\lim _{n, m \rightarrow \infty} \omega_{\lambda}^{G}\left(S x_{n}, S x_{m}, S x_{m}\right)+\lim _{k, m \rightarrow \infty} \omega_{\lambda}^{G}\left(S x_{k}, S x_{m}, S x_{m}\right)\right),
\end{aligned}
$$

hence, we have

$$
\lim _{n, m, k \rightarrow \infty} \omega_{\lambda}^{G}\left(S x_{n}, S x_{m}, S x_{k}\right)=0 .
$$


By Eq. (4.20), the sequence $\left\{S x_{n}\right\}_{n \in \mathbb{N}}$ is a modular $G$-Cauchy sequence in $S\left(X_{\omega}\right)$. Because of completeness of $S\left(X_{\omega}\right), S x_{n} \rightarrow v$ and there exists $u \in X_{\omega}$ such that $S u=v$. Hence, inequality (4.3) becomes $\omega_{\lambda}^{G}\left(S x_{n}, T u, T u\right)=\omega_{\lambda}^{G}\left(T x_{n-1}, T u, T u\right)$, so

$$
\omega_{\lambda}^{G}\left(S x_{n}, T u, T u\right) \leq k \max \left\{\begin{array}{l}
\omega_{\lambda}^{G}\left(S x_{n-1}, S u, S u\right), \omega_{\lambda}^{G}\left(T x_{n-1}, T x_{n-1}, S u\right), \\
\omega_{\lambda}^{G}(T u, T u, S u), \omega_{\lambda}^{G}(S u, T u, S u), \\
\omega_{\lambda}^{G}\left(S u, T x_{n-1}, S u\right), \omega_{\lambda}^{G}(S u, T u, T u), \\
\omega_{\lambda}^{G}\left(T u, S x_{n-1}, T u\right), \omega_{\lambda}^{G}\left(T x_{n-1}, S x_{n-1}, S u\right)
\end{array}\right\}
$$

so that

$$
\omega_{\lambda}^{G}\left(S x_{n}, T u, T u\right) \leq k \max \left\{\begin{array}{l}
\omega_{\lambda}^{G}\left(S x_{n-1}, S u, S u\right), \omega_{\lambda}^{G}\left(S x_{n}, S x_{n}, S u\right), \\
\omega_{\lambda}^{G}(T u, T u, S u), \omega_{\lambda}^{G}(S u, T u, S u), \\
\omega_{\lambda}^{G}\left(S u, S x_{n}, S u\right), \omega_{\lambda}^{G}(S u, T u, T u), \\
\omega_{\lambda}^{G}\left(T u, S x_{n-1}, T u\right), \omega_{\lambda}^{G}\left(S x_{n}, S x_{n-1}, S u\right)
\end{array}\right\}
$$

as $n \rightarrow \infty$, and $S u=v$ we obtain

$$
\omega_{\lambda}^{G}(v, T u, T u) \leq k \max \left\{\omega_{\lambda}^{G}(v, T u, T u), \omega_{\lambda}^{G}(v, v, T u)\right\}, \quad \forall \lambda>0 .
$$

We consider the following cases.

Case 1. If $k \max \left\{\omega_{\lambda}^{G}(v, T u, T u), \omega_{\lambda}^{G}(v, v, T u)\right\}=\omega_{\lambda}^{G}(v, T u, T u)$, then inequality (4.23) becomes

$$
\omega_{\lambda}^{G}(v, T u, T u) \leq k \omega_{\lambda}^{G}(v, T u, T u)
$$

which implies that

$$
(1-k) \omega_{\lambda}^{G}(v, T u, T u) \leq 0 \quad \forall \lambda>0, k \in(0,1),
$$

hence $T u=v=S u$.

Case 2. If $k \max \left\{\omega_{\lambda}^{G}(v, T u, T u), \omega_{\lambda}^{G}(v, v, T u)\right\}=\omega_{\lambda}^{G}(v, v, T u)$, then inequality (4.23) becomes

$$
\omega_{\lambda}^{G}(v, T u, T u) \leq k \omega_{\lambda}^{G}(v, v, T u)
$$

using condition (4) of Proposition 2.2, and Definition 2.11, we get

$$
\begin{aligned}
\omega_{\lambda}^{G}(v, T u, T u) & \leq k\left(\omega_{\frac{\lambda}{2}}^{G}(v, T u, T u)+\omega_{\frac{\lambda}{2}}^{G}(T u, v, T u)\right) \\
& \leq k\left(C_{2} \omega_{\lambda}^{G}(v, T u, T u)+C_{2} \omega_{\lambda}^{G}(v, T u, T u)\right) \\
& =2 k C_{2} \omega_{\lambda}^{G}(v, T u, T u),
\end{aligned}
$$


which implies that

$$
\left(1-2 k C_{2}\right) \omega_{\lambda}^{G}(v, T u, T u) \leq 0,
$$

for some $C_{2} \in\left(0, \frac{1}{k}\right)$, hence $\omega_{\lambda}^{G}(v, T u, T u) \leq 0$, this implies that $T u=v=S u$, which shows that $v$ is a point of coincidence of $T$ and $S$. Now suppose that $v$ is not a point of coincidence of $T$ and $S$, then there exists another point of coincidence (say $v^{*}$ ) of $T$ and $S$ such that $T u^{*}=v^{*}=S u^{*}$. For $\lambda>0, \omega_{\lambda}^{G}\left(S u, S u^{*}, S u^{*}\right)=\omega_{\lambda}^{G}\left(T u, T u^{*}, T u^{*}\right)$, so from inequality (4.3), we obtain

$$
\omega_{\lambda}^{G}\left(S u, S u^{*}, S u^{*}\right) \leq k \max \left\{\begin{array}{l}
\omega_{\lambda}^{G}\left(S u, S u^{*}, S u^{*}\right), \omega_{\lambda}^{G}\left(T u, T u, S u^{*}\right), \\
\omega_{\lambda}^{G}\left(T u^{*}, T u^{*}, S u^{*}\right), \omega_{\lambda}^{G}\left(S u^{*}, T u^{*}, S u^{*}\right), \\
\omega_{\lambda}^{G}\left(S u^{*}, T u, S u^{*}\right), \omega_{\lambda}^{G}\left(S u^{*}, T u^{*}, T u^{*}\right), \\
\omega_{\lambda}^{G}\left(T u^{*}, S u, T u^{*}\right), \omega_{\lambda}^{G}\left(T u, S u, S u^{*}\right)
\end{array}\right\}
$$

hence

$$
\omega_{\lambda}^{G}\left(S u, S u^{*}, S u^{*}\right) \leq k \max \left\{\omega_{\lambda}^{G}\left(S u, S u^{*}, S u^{*}\right), \omega_{\lambda}^{G}\left(S u, S u, S u^{*}\right)\right\}
$$

If $k \max \left\{\omega_{\lambda}^{G}\left(S u, S u^{*}, S u^{*}\right), \omega_{\lambda}^{G}\left(S u, S u, S u^{*}\right)\right\}=k \omega_{\lambda}^{G}\left(S u, S u^{*}, S u^{*}\right)$, then inequality (4.30) becomes

$$
(1-k) \omega_{\lambda}^{G}\left(S u, S u^{*}, S u^{*}\right) \leq 0, \quad k \in(0,1)
$$

which implies that $S u=S u^{*}$.

Again, if $k \max \left\{\omega_{\lambda}^{G}\left(S u, S u^{*}, S u^{*}\right), \omega_{\lambda}^{G}\left(S u, S u, S u^{*}\right)\right\}=k \omega_{\lambda}^{G}\left(S u, S u, S u^{*}\right)$, then using condition (4) of Proposition 2.2, and Definition 2.11, we get

$$
\begin{aligned}
\omega_{\lambda}^{G}\left(S u, S u^{*}, S u^{*}\right) & \leq k\left(\omega_{\frac{\lambda}{2}}^{G}\left(S u, S u^{*}, S u^{*}\right)+\omega_{\frac{\lambda}{2}}^{G}\left(S u^{*}, S u, S u^{*}\right)\right) \\
& \leq k\left(C_{2} \omega_{\lambda}^{G}\left(S u, S u^{*}, S u^{*}\right)+C_{2} \omega_{\lambda}^{G}\left(S u, S u^{*}, S u^{*}\right)\right) \\
& =2 k C_{2} \omega_{\lambda}^{G}\left(S u, S u^{*}, S u^{*}\right),
\end{aligned}
$$

which implies that

$$
\left(1-2 k C_{2}\right) \omega_{\lambda}^{G}\left(S u, S u^{*}, S u^{*}\right) \leq 0
$$

for some $C_{2} \in\left(0, \frac{1}{k}\right)$, hence $\omega_{\lambda}^{G}\left(S u, S u^{*}, S u^{*}\right) \leq 0$. Therefore, $S u=S u^{*}$, which shows that $v$ is a unique point of coincidence of $T$ and $S$. By Proposition 2.1, $v$ is a common unique point of $T$ and $S$.

We give some examples here.

Example 4.1 Let $X=[0,1] \cup\{\infty\}$, and for $\lambda>0$, define $\omega_{\lambda}^{G}: X \times X \times X \rightarrow[0, \infty)$ by $\omega_{\lambda}^{G}(x, y, z)=\frac{1}{\lambda} \max \{|x-y|,|y-z|,|x-z|\}$ and $T, S: X_{\omega^{G}} \rightarrow X_{\omega^{G}}$ such that $T x=\frac{1}{2}$ and 
$S x=\frac{1}{2}-x$. Evidently, the range of $T$ contains the range of $S, R(S) \subseteq R(T)$ and $S\left(X_{\omega^{G}}\right)$ is a complete subspace of $X_{\omega^{G}}$, also $T$ and $S$ has a unique point of coincidence at $x=0$ in $X$. Furthermore, $T$ and $S$ are weakly compatible. But $\omega_{\lambda}^{G}(T x, T y, T z)=0$, so $T$ satisfies inequality (4.3).

Example 4.2 Let $X=\mathbb{R}^{r} \cup\{\infty\}$ for $r \geq 1$. Define $G: \mathbb{R}^{r} \times \mathbb{R}^{r} \times \mathbb{R}^{r} \rightarrow[0, \infty)$ by $G(x, y, z)=$ $\frac{1}{3} \max \{|x-y|,|y-z|,|x-z|\}$ for all distinct $x, y, z \in \mathbb{R}^{r}$ and 0 for $x=y=z$, where $|x-y|=$ $\left(\sum_{k=1}^{r}\left(x_{k}-y_{k}\right)^{2}\right)^{\frac{1}{2}}, x=\left(x_{1}, x_{2}, \ldots, x_{r}\right), y=\left(y_{1}, y_{2}, \ldots, y_{r}\right)$ and $z=\left(z_{1}, z_{2}, \ldots, z_{r}\right)$. For any $\lambda>0$, let $\omega_{\lambda}^{G}(x, y, z)=\frac{G(x, y, z)}{3 \lambda}$ for all $x, y, z \in \mathbb{R}^{r}$. Suppose that two mappings $T, S: \mathbb{R}^{r} \rightarrow \mathbb{R}^{r}$ defined by $T x=\frac{x}{6}$ and $S x=\frac{x}{3}$. Indeed observe that $R(S) \subseteq R(T)$ and $S\left(X_{\omega^{G}}\right)$ is a complete subspace of $X, T(S(0))=S(T(0))$ also $T$ and $S$ has a unique point of coincidence at $x=0$ in $X$, i.e. $u=T(0)=S(0)=0$, so that the common unique fixed point of $T$ and $S$ is 0 . Now

$$
\begin{aligned}
& \omega_{\lambda}^{G}(T x, T y, T z) \\
& =\frac{1}{6 \lambda} \max \left\{\left(\sum_{k=1}^{r}\left(x_{k}-y_{k}\right)^{2}\right)^{\frac{1}{2}},\left(\sum_{k=1}^{r}\left(y_{k}-z_{k}\right)^{2}\right)^{\frac{1}{2}},\left(\sum_{k=1}^{r}\left(x_{k}-z_{k}\right)^{2}\right)^{\frac{1}{2}}\right\} \\
& =\frac{1}{2}\left\{\frac{1}{3 \lambda} \max \left\{\left(\sum_{k=1}^{r}\left(x_{k}-y_{k}\right)^{2}\right)^{\frac{1}{2}},\left(\sum_{k=1}^{r}\left(y_{k}-z_{k}\right)^{2}\right)^{\frac{1}{2}},\left(\sum_{k=1}^{r}\left(x_{k}-z_{k}\right)^{2}\right)^{\frac{1}{2}}\right\}\right\} \\
& =\frac{1}{2} \omega_{\lambda}^{G}(S x, S y, S z),
\end{aligned}
$$

so that

$$
\omega_{\lambda}^{G}(T x, T y, T z) \leq \frac{1}{2} \max \left\{\begin{array}{c}
\omega_{\lambda}^{G}(S x, S y, S z), \omega_{\lambda}^{G}(T x, T x, S z), \\
\omega_{\lambda}^{G}(T y, T y, S y), \omega_{\lambda}^{G}(S y, T z, S z), \\
\omega_{\lambda}^{G}(S y, T x, S y), \omega_{\lambda}^{G}(S z, T z, T z), \\
\omega_{\lambda}^{G}(T z, S x, T z), \omega_{\lambda}^{G}(T x, S x, S y)
\end{array}\right\}
$$

hence all the conditions of Theorem 4.2 are satisfied. Thus $T$ and $S$ has a unique point of coincidence at $x=0$ in $X$.

Corollary 4.3 Let $\left(X, \omega^{G}\right)$ be a modular G-metric space satisfying a $\Delta_{3}$-type condition and $T, S: X_{\omega^{G}} \rightarrow X_{\omega^{G}}$ be two self-mappings for which $T\left(X_{\omega^{G}}\right) \subseteq S\left(X_{\omega^{G}}\right)$, where $S\left(X_{\omega^{G}}\right)$ is a G-complete subspace of $X_{\omega^{G}}$. Suppose that, for all $\lambda>0$, the following condition holds:

$$
\omega_{\lambda}^{G}(T x, T y, T z) \leq k \omega_{\lambda}^{G}(S x, S y, S z)
$$

where $k<1$, for all $x, y, z \in X_{\omega^{G}}$. Then $T, S$ has a unique coincidence point in $X_{\omega^{G}}$. Moreover, if $T$ and $S$ are weakly compatible, then $T$ and $S$ have a unique common fixed point in $X_{\omega} G$.

Proof Let $x_{0} \in X_{\omega^{G}}$ be an arbitrary point. Since $T\left(X_{\omega^{G}}\right) \subseteq S\left(X_{\omega^{G}}\right)$, there exists $x_{1} \in X_{\omega^{G}}$ such that $T x_{0}=S x_{1}$. Continuing in this way we have $T x_{n}=S x_{n+1}$, for $n \in \mathbb{N}$. So for any 
$\lambda>0, \omega_{\lambda}^{G}\left(S x_{n+1}, S x_{n}, S x_{n}\right) \leq \omega_{\lambda}^{G}\left(T x_{n}, T x_{n-1}, T x_{n-1}\right)$. From inequality (4.36), we have

$$
\omega_{\lambda}^{G}\left(S x_{n+1}, S x_{n}, S x_{n}\right) \leq k \omega_{\lambda}^{G}\left(S x_{n}, S x_{n-1}, S x_{n-1}\right), \quad k \in(0,1)
$$

Theorem 4.2 tells us that $T$ and $S$ have a unique common fixed point in $X_{\omega^{G}}$.

Corollary 4.4 Let $\left(X, \omega^{G}\right)$ be a modular G-metric space satisfying a $\Delta_{3}$-type condition, such that $C_{2}+C_{4} \in\left(0, \frac{1}{k}\right)$ and $T, S: X_{\omega^{G}} \rightarrow X_{\omega^{G}}$ be two self-mappings for which $T^{m}\left(X_{\omega^{G}}\right) \subseteq$ $S^{m}\left(X_{\omega^{G}}\right)$, where $S^{m}\left(X_{\omega^{G}}\right)$ is a G-complete subspace of $X_{\omega^{G}}$. Suppose that, for all $\lambda>0$, the following condition holds:

$$
\omega_{\lambda}^{G}\left(T^{m} x, T^{m} y, T^{m} z\right) \leq k \max \left\{\begin{array}{c}
\omega_{\lambda}^{G}\left(S^{m} x, S^{m} y, S^{m} z\right), \omega_{\lambda}^{G}\left(T^{m} x, T^{m} x, S^{m} z\right), \\
\omega_{\lambda}^{G}\left(T^{m} y, T^{m} y, S^{m} y\right), \omega_{\lambda}^{G}\left(S^{m} y, T^{m} z, S^{m} z\right), \\
\omega_{\lambda}^{G}\left(S^{m} y, T^{m} x, S^{m} y\right), \omega_{\lambda}^{G}\left(S^{m} z, T^{m} z, T^{m} z\right), \\
\omega_{\lambda}^{G}\left(T^{m} z, S^{m} x, T^{m} z\right), \omega_{\lambda}^{G}\left(T^{m} x, S^{m} x, S^{m} y\right)
\end{array}\right\},
$$

where $k<1$ for all $x, y, z \in X_{\omega} G$. Then $T$, S has a unique coincidence point in $X$. Moreover, if $T$ and $S$ are weakly compatible, then $T$ and $S$ have a unique common fixed point in $X_{\omega^{G}}$ for some positive integer $m \geq 1$.

Proof By Theorem 4.2, $T^{m} u=S^{m} u=v$. Hence $v$ is a common unique point of $T^{m}$ and $S^{m}$.

\section{Acknowledgements}

The authors wish to thank the editor and the anonymous referees for their useful comments and suggestions. This research was initiated when the first author was visiting the Abdus Salam School of Mathematical Sciences (ASSMS), Lahore Pakistan as a Postdoctoral fellow. The first author is grateful to ASSMS for hospitality.

\section{Funding}

This research was funded by Basque Government (IT1207-19).

\section{Availability of data and materials}

Not applicable.

\section{Competing interests}

The authors declare that they have no competing interests.

\section{Authors' contributions}

All authors contributed equally to the writing of this paper. All authors read and approved the final manuscript.

\section{Author details}

'Functional Analysis and Optimization Research Group Laboratory (FANORG), Department of Mathematics, School of Physical Sciences, Federal University of Technology Owerri, P.M.B. 1526, Owerri, Imo State, Nigeria. ${ }^{2}$ Institute of Research and Development of Processes, University of the Basque Country, Campus of Leioa (Bizkaia)—P.O. Box 644—Bilbao, Barrio Sarriena, 48940 Leioa, Spain. ${ }^{3}$ Department of Mathematics, Government College University, Katchery Road, Lahore 54000, Pakistan. ${ }^{4}$ Department of Medical Research, China Medical University Hospital, China Medical University, Taichung 40402, Taiwan.

\section{Publisher's Note}

Springer Nature remains neutral with regard to jurisdictional claims in published maps and institutional affiliations.

Received: 18 June 2021 Accepted: 16 September 2021 Published online: 02 October 2021

\section{References}

1. Abbas, M., Nazir, T., Saadati, R.: Common fixed point results for three maps in G-metric spaces. Filomat 25(4), 1-17

(2011) 
2. Abbas, M., Nazir, T., Shatanawi, W., Mustafa, Z:: Fixed and related fixed point theorems for three maps in G-metric spaces. Hacet. J. Math. Stat. 41(2), 291-306 (2012)

3. Abd-Elhamed, G.M.: Fixed point results for $(\beta, \alpha)$-implicit contractions in two generalized $b$-metric spaces. J. Nonlinear Sci. Appl. 14(1), 39-47 (2021)

4. Abdou, A.A.N.: Some fixed point theorems in modular metric spaces. J. Nonlinear Sci. Appl. 9, 4381-4387 (2016)

5. Abobaker, H., Ryan, R.A.: Modular metric spaces. Ir. Math. Soc. Bull. 21, 35-44 (2017)

6. Abusalim Sahar, M., Fadail Zaid, M.: New coupled and common coupled fixed point results with generalized c-distance on cone b-metric spaces. J. Math. Comput. Sci. 25(3), 209-218 (2022)

7. Agarwal, R.P., Karapinar, E.: Remarks on some coupled fixed point theorems in G-metric spaces. Fixed Point Theory Appl. 2013, 2 (2013)

8. Agarwal, R.P., Karapinar, E., O’Regan, D., Roldan-Lopez-de-Hierro, A.F.: Fixed Point Theory in Metric Type Spaces. Springer, Cham (2015)

9. Agarwal, R.P., Karapinar, E., Roldan-Lopez-de-Hierro, A.F.: Fixed point theorems in quasimetric spaces and applications to multidimensional fixed points on G-metric spaces. J. Nonlinear Convex Anal. 16, 1787-1816 (2015)

10. Aggarwal, M., Chugh, R., Kamal, R.: Suzuki-type fixed point results in G-metric spaces and applications. Int. J. Comput. Appl. 47, 14-17 (2012)

11. Amini-Harandi, A.: A fixed point theory for generalized quasicontraction maps in vector modular spaces. Comput. Math. Appl. 61, 1891-1897 (2011)

12. Asadi, M., Karapinar, E., Salimi, P.: A new approach to G-metric and related fixed point theorems. J. Inequal. Appl. 2013, $454(2013)$

13. Azadifar, B.: Integral-type contraction in modular metric spaces. J. Inequal. Appl. 2013, 482 (2013)

14. Azadifar, B., Maramaei, M., Sadeghi, G.: On the modular G-metric spaces and fixed point theorems. J. Nonlinear Sci. Appl. 6, 293-304 (2013)

15. Azadifar, B., Maramaei, M., Sadeghi, G.: Common fixed point theorems in modular G-metric spaces. J. Nonlinear Anal. Appl. 2013, Article ID jnaa-00175 (2013)

16. Banach, S.: Sur les operations dans les ensembles abstracts et leure application aux equations integrals. Fundam. Math. 3, 133-181 (1922)

17. Bhaskar, I.G., Lakshmikantham, V.: Fixed point theorems in partially ordered metric spaces and applications. Nonlinear Anal. 65, 1379-1393 (2006)

18. Chaipunya, P., Cho, Y.J., Kumam, P.: Geraghty-type theorems in modular metric spaces with application to partial differential equation. Adv. Differ. Equ. 2012, 83 (2012)

19. Chaipunya, P., Mongkolkeha, C., Sintunavarat, W., Kumam, P.: Fixed point theorems for multivalued mappings in modular metric spaces. Abstr. Appl. Anal. 2012, Article ID 503504 (2012)

20. Chistyakov, V.V.: Metric modular spaces, I. Basic concepts. Nonlinear Anal., Theory Methods Appl. 72, 1-14 (2010)

21. Chistyakov, V.V.: Metric modular spaces, II. Applications to superposition operators. Nonlinear Anal., Theory Methods Appl. 72, 15-30 (2010)

22. Chistyakov, V.V.: Metric Modular Spaces, Theory and Applications. Springer, Cham (2015)

23. Dhage, B.C.: Generalized metric space and mapping with fixed point. Bull. Calcutta Math. Soc. 84, 329-336 (1992)

24. Gahler, S.: 2-metrische Raume und ihre topologische struktur. Math. Nachr. 26, 665-667 (1966)

25. Hashemi, E., Ghaemi, B.M.: Ekeland's variational principle in complete quasi-G-metric spaces. J. Nonlinear Sci. Appl. 12(3), 184-191 (2019)

26. Hussain, N., Ahmad, J., Azam, A.: Generalized fixed point theorems for multi-valued $\alpha$ - $\psi$-contractive mappings. J. Inequal. Appl. 2014, 348 (2014)

27. Hussain, N., Kutbi, M.A., Sultana, N., lqbal, I.: Weak contractive integral inequalities and fixed points in modular metric spaces. J. Inequal. Appl. 2016, 89 (2016)

28. Hussain, S.: Non-unique fixed point theorems in modular metric spaces. Symmetry 11, 549 (2019)

29. Jleli, M., Samet, B.: Remarks on G-metric spaces and fixed point theorems. Fixed Point Theory Appl. 2012,210 (2012)

30. Karapinar, E., Agarwal, R.P.: Further fixed point results on G-metric spaces. Fixed Point Theory Appl. 2013, 154 (2013)

31. Kittipong, S., Satit, S.: Some fixed point theorems in complex valued metric spaces. Fixed Point Theory Appl. 2012 $189(2012)$

32. Kumar, M., Arora, S., Imdad, M., Alfagih Waleed, M.: Coincidence and common fixed point results via simulation functions in G-metric spaces. J. Math. Comput. Sci. 19(4), 288-300 (2019)

33. Lateef, D.: Fisher type fixed point results in controlled metric spaces. J. Math. Comput. Sci. 20(3), 234-240 (2020)

34. Liu, Z., Li, X., Kang, S.M., Cho, Y.J.: Fixed point theorems for mappings satisfying contractive conditions of integral-type and applications. Fixed Point Theory Appl. 2011, 64 (2011)

35. Mongkolkeha, C., Sintunavarat, W., Kumam, P.: Fixed point theorem for contraction mappings in modular spaces. Fixed Point Theory Appl. 2011, 93 (2011)

36. Musielak, J.: Orlicz Spaces and Modular Spaces. Lecture Notes in Math., vol. 1034. Springer, Berlin (1983)

37. Mustafa, Z:: A new structure for generalized metric spaces with applications to fixed point theory. Ph.D. thesis, The University of Newcastle, Australia (2005)

38. Mustafa, Z.: Common fixed points of weakly compatible mappings in G-metric spaces. Appl. Math. Sci. 6(92), 4589-4600 (2012)

39. Mustafa, Z:: Some new common fixed point theorems under strict contractive conditions in G-metric spaces. J. Appl. Math. 2012, Article ID 248937 (2012)

40. Mustafa, Z., Awawdeh, F., Shatanawi, W.: Fixed point theorem for expansive mappings in G-metric spaces. Int. J. Contemp. Math. Sci. 5(50), 2463-2472 (2010)

41. Mustafa, Z., Aydi, H., Karapinar, E.: On common fixed points in G-metric spaces using (E.A) property. Comput. Math. Appl. 64(6), 1944-1956 (2012)

42. Mustafa, Z., Jaradat, M.M.M.: Some remarks concerning D*-metric spaces. J. Math. Comput. Sci. 22(2), 128-130 (2021)

43. Mustafa, Z., Khandagji, M., Shatanawi, W.: Fixed point results on complete G-metric spaces. Studia Sci. Math. Hung. 48(3), 304-319 (2011)

44. Mustafa, Z., Obiedat, H., Awawdeh, F.: Some fixed point theorem for mapping on complete G-metric spaces. Fixed Point Theory Appl. 2008, Article ID 189870 (2008) 
45. Mustafa, Z., Sims, B.: Some remarks concerning D-metric spaces. In: Proceedings of the International Conference on Fixed Point Theory and Applications, Valencia (Spain), p. 198 (2003)

46. Mustafa, Z., Sims, B.: A new approach to generalized metric spaces. J. Nonlinear Convex Anal. 7(2), 289-297 (2006)

47. Mustafa, Z., Sims, B.: Fixed point theorems for contractive mappings in complete G-metric spaces. Fixed Point Theory Appl. 2009, Article ID 917175 (2009)

48. Mustafa, Z., Wasfi, S., Bataineh, M.: Existence of fixed point results in G-metric spaces. Int. J. Math. Math. Sci. 2009, Article ID 283028 (2009)

49. Mutlu, A., Ozkan, K., Gurdal, U.: A new fixed point theorem in modular metric spaces. Int. J. Anal. Appl. 4, 472-483 (2018)

50. Nadler, S.B.: Multi-valued contraction mappings. Pac. J. Math. 30(2), 475-488 (1969)

51. Nakano, H.: On the stability of functional equations. Aequ. Math. 77, 33-88 (2009)

52. Okeke, G.A., Bishop, S.A., Khan, S.H.: Iterative approximation of fixed point of multivalued $\rho$-quasi-nonexpansive mappings in modular function spaces with applications. J. Funct. Spaces 2018, Article ID 1785702 (2018)

53. Okeke, G.A., Francis, D.: Fixed point theorems for Geraghty-type mappings applied to solving nonlinear Volterra-Fredholm integral equations in modular G-metric spaces. Arab J. Math. Sci. 27(2), 214-234 (2021)

54. Okeke, G.A., Francis, D., de la Sen, M.: Some fixed point theorems for mappings satisfying rational inequality in modular metric spaces with applications. Heliyon 6, e04785 (2020)

55. Okeke, G.A., Khan, S.H.: Approximation of fixed point of multivalued $\rho$-quasi-contractive mappings in modular function spaces. Arab J. Math. Sci. 26(1/2), 75-93 (2020)

56. O'Regan, D.: Essentiality and fixed point results for Eilenberg-Montgomery type maps. J. Math. Comput. Sci. 22(4), 392-398 (2021)

57. Orlicz, W.: Collected Papers, Vols. I, II. PWN, Warsaw (1988)

58. Ramesh, D.K.: Generalized rational inequalities in complex valued metric spaces. J. Comput. Math. 1(2), 118-129 (2017)

59. Reich, S.: Fixed points of contractive functions. Boll. Unione Mat. Ital. 5, 26-42 (1972)

60. Reich, S.: Some fixed point problems. Atti Accad. Naz. Lincei, Rend. Cl. Sci. Fis. Mat. Nat. 57, 194-198 (1974)

61. Rhoades, B.E.: Two fixed point theorems for mappings satisfying a general contractive condition in integral-type. Int. J. Math. Sci. 63, 4007-4013 (2003)

62. Samet, B., Vetro, C., Vetro, F.: Remarks on G-metric spaces. Int. J. Anal. 2013, Article ID 917158 (2013)

63. Shatanawi, W., Bataihah, A., Pitea, A.: Fixed and common fixed point results for cyclic mappings of $\Omega$-distance J. Nonlinear Sci. Appl. 9, 727-735 (2016)

64. Swati, A., Gairola, U.C.: Generalized Suzuki type $\alpha$-Z्Z-contraction in b-metric space. J. Nonlinear Sci. Appl. 13(4), 212-222 (2020)

65. Zhou, C., Wang, S., Ciric, L., Alsulami, S.M.: Generalized probabilistic metric spaces and fixed point theorems. Fixed Point Theory Appl. 2014, 91 (2014)

\section{Submit your manuscript to a SpringerOpen ${ }^{\circ}$ journal and benefit from:}

- Convenient online submission

- Rigorous peer review

- Open access: articles freely available online

- High visibility within the field

- Retaining the copyright to your article

Submit your next manuscript at $\boldsymbol{\nabla}$ springeropen.com 